Rogério de Oliveira

\title{
Mapas acoplados e aplicações: processamento de imagens, auto-organização e processamento simbólico
}

Texto apresentado à Escola Politécnica da Universidade de São Paulo para obtenção do Título de Doutor em Engenharia. 
Rogério de Oliveira

\section{Mapas acoplados e aplicações: processamento de imagens, auto-organização e processamento simbólico}

Texto apresentado à Escola Politécnica da Universidade de São Paulo para obtenção do Título de Doutor em Engenharia.

Área de concentração:

Engenharia de Sistemas

Orientador:

Luiz Henrique Alves Monteiro 
Este exemplar foi revisado e alterado em relação à versão original, sob responsabilidade única do autor e com a anuência de seu orientador

São Paulo, 15 de fevereiro de 2004.

Assinatura do autor

Assinatura do orientador

Ficha Catalográfica

Oliveira, Rogério de

Mapas acoplados e aplicações:

processamento de imagens, auto-organização e processamento simbólico. / Rogério de Oliveira - ed. rev. - São Paulo, 2004. 122 p.

Tese (Doutorado) - Escola Politécnica da Universidade de São Paulo. Departamento de Engenharia de Telecomunicações e Controle.

1. Sistemas dinâmicos. 2. Processamento de imagens. 3. Computabilidade e complexidade. I. Universidade de São Paulo. Escola Politécnica. Departamento de Engenharia de Telecomunicações e Controle. II. t. 
A minha esposa Lucimara

e meu filho Daniel, 


\section{Agradecimentos}

Primeiramente agradeço ao meu orientador, Luiz Henrique Alves Monteiro, por ter tornado possível este trabalho e por haver conciliado exigência e liberdade na condução desta pesquisa.

Agradeço também a todos os professores e colegas, que contribuiram e incentivaram a realização desta tese, talvez muitas vezes sem se darem conta disso. Em especial, agradeço aos professores e colegas Claudio Garcia, Emílio Del Moral Hernandez, Gina Maira Barbosa de Oliveira, José Roberto Castilho Piqueira, Keich Hirama, Márcio Lobo, Mauro Pereira, Nizam Omar, Rogério Neves, Vilmar Pedro Votre e Zsolt Laszlo Kovacs.

Por último agradeço, à Universidade Presbiteriana Mackenzie e ao Departamento de Engenharia de Telecomunicações e Controle da Escola Politécnica, entidades que possibilitaram a elaboração deste estudo. 


\section{Resumo}

Investigamos algumas capacidades computacionais de sistemas constituídos de mapas acoplados. Particularmente, exploramos o uso desses sistemas no tratamento de três problemas: a identificação de simetria de reflexão em imagens planas; a formação de clusters de elementos síncronos em redes com topologias do tipo small-worlds; e a construção de figuras que obedecem a uma regra de composição.

Para a identificação de simetria, motivados por modelos biológicos, construímos uma rede de mapas em que, acoplamentos locais e globais permitem verificar a simetria de reflexão de uma imagem plana através do sincronismo dos elementos do sistema. Em particular, esse sistema apresenta a habilidade de não requerer sua reinicialização para novas identificações e permite, assim, a identificação de simetrias em cenas que se modificam no tempo.

Sistemas estendidos de mapas acoplados são, em geral, construídos conectando-se todos os elementos ou pela formação de uma malha uniforme de conexões. A dinâmica desses sistemas pode apresentar a formação de grupos de elementos síncronos. Esse comportamento de auto-organização pode ser encontrado em diversos sistemas complexos reais que, entretanto e mais comumente, exibem topologias de conexões não uniformes entre seus elementos. Mostramos aqui, a capacidade de mapas acoplados, em diferentes topologias de small-worlds, exibirem a formação de grupos de elementos síncronos, com um número de conexões próximo ao das malhas com acoplamento local mas com uma significativa redução da distância média entre os elementos da rede.

Por último, consideramos o uso de sistemas de mapas como sistemas programáveis. Normalmente, para formação de padrões e figuras no plano, sistemas de funções iteradas são empregados com um conjunto fixo de contrações lineares no plano. Aqui, mostramos a possibilidade do uso de mapas mais gerais na produção de tais padrões e figuras, incluindo estruturas biológicas e fractais. Funções de troca são empregadas para alterar a dinâmica do sistema segundo ou o contexto ou o estado, e fornecem, desse modo, uma forma de programação. 


\section{Abstract}

We investigated some computational abilities of systems composed by coupled maps. Here, we explored the use of those systems in dealing with three problems: the identification of reflection symmetry in bidimensional images; the appearing of clusters of synchronous elements in networks with small-worlds topologies; and in constructing figures obeying a composition rule.

For the symmetry identification problem, we were motivated by biological models to built a network of coupled maps, with local and global couplings, that verify reflection symmetry of plane images through the synchronism of the elements from the system. In matter, this system presents the ability to perform a new identification without reinitializing the system. This feature allows the identification of symmetries in scenes that can change during the time.

In general extended coupled map systems have all elements connected, or the connections lying over a uniform lattice. The dynamics of these systems can present the formation of clusters with synchronous elements. Such auto-organization behavior can be found in several actual complex systems. However, more commonly, these systems do not exhibit uniform connections among their elements. Here, we investigated the capacity of coupled map systems, in different topologies of small-worlds, exhibiting the formation of clusters with synchronous elements, by using a number of connections close to the number in regular lattices but with a significant reduction of the mean distance among their elements.

Last we considered the use of systems of maps as programmable systems. Usually, for formation of patterns and geometric figures in the plan, iterated function systems work with a fixed set of linear contractions in the plan. Here, we showed that is possible to use more general maps to the production of patterns and geometric figures, and biological patterns and fractals are generated. Shift functions are used to change the dynamics of the map system due to either the context or the state, giving a way of programming the system. 


\section{Sumário}

\section{Lista de Figuras}

\section{Lista de Tabelas}

1 Introdução 1

1.1 Detecção de simetria . . . . . . . . . . . . . . . . 2

1.2 Auto-organização em redes não regulares . . . . . . . . . . . . . . 2

1.3 Sistemas programáveis e a produção de figuras e padrões no plano . . . . . 3

1.4 Organização do trabalho . . . . . . . . . . . . . . . . 4

2 Mapas, acoplamentos e sincronismo: conceitos preliminares 5

2.1 Sistemas dinâmicos em tempo discreto . . . . . . . . . . . 5

2.2 Mapas, órbitas e pontos periódicos . . . . . . . . . 7

2.3 Mapas e campos vetoriais . . . . . . . . . . . . 8

2.4 Mapas parametrizados e bifurcações . . . . . . . . . . . . . . 10

2.5 Dinâmica de uma função e caos . . . . . . . . . . . . . . . . . 12

2.6 Sincronismo e acoplamento . . . . . . . . . . . . . 16

2.7 Mapas acoplados e sistemas estendidos . . . . . . . . . . . . 22

2.8 Conclusão e sumário . . . . . . . . . . . . . . . . . . . . 29

3 Processamento de imagens: detecção de simetria 30

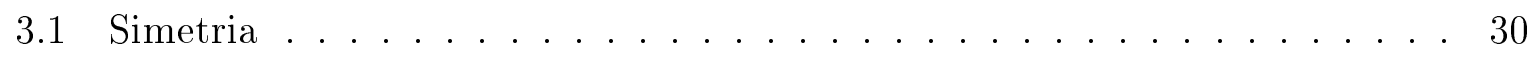

3.2 Descrição do modelo . . . . . . . . . . . . . . . . 34 
3.3 Simulações e resultados . . . . . . . . . . . . . . . . . 37

3.4 Discussão e modelos biológicos . . . . . . . . . . . . . . . . 44

3.5 Conclusão e sumário . . . . . . . . . . . . . . . . . . 47

4 Sistemas auto-organizáveis $\quad 48$

4.1 Redes complexas . . . . . . . . . . . . . . . . . . 49

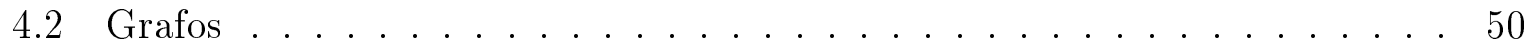

4.3 Medidas para redes complexas . . . . . . . . . . . . . . . 51

4.4 Propriedades dos modelos . . . . . . . . . . . . . . . 52

4.5 Small-worlds . . . . . . . . . . . . . . . . 56

4.6 Mapas acoplados em redes small-world . . . . . . . . . . . . . 59

4.7 Simulações e resultados . . . . . . . . . . . . . . . . 65

4.8 Conclusão e sumário . . . . . . . . . . . . . . . . . . 72

5 Computabilidade e processamento simbólico $\quad 73$

5.1 Máquina de Turing . . . . . . . . . . . . . . . . . . 74

5.2 Computação universal . . . . . . . . . . . . . . 76

5.3 Computabilidade por sistemas de portas lógicas $\ldots \ldots \ldots 78$

5.4 Máquinas de Turing e mapas lineares em intervalos . . . . . . . . . . 81

5.5 Máquinas de Turing e o mapa do padeiro . . . . . . . . . . 85

5.6 Modelo para computação não convencional . . . . . . . . . . . . 87

$5.7 \quad$ L-sistemas . . . . . . . . . . . . . . . . . . 89

5.8 Processamento simbólico e mapas . . . . . . . . . . . . . . . 91

5.9 Conclusão e sumário . . . . . . . . . . . . . . . . 102

6 Conclusão 105

$\begin{array}{ll}\text { Referências } & 107\end{array}$ 


\section{Lista de Figuras}

2.1 A seqüência mostrada na figura (a), uma órbita $\left\{x_{t}\right\}$ de período 4 , não pode ser obtida através de um mapa autônomo de ordem 1, pois apresenta pontos $\tilde{x}_{t}$ (os pontos de maior valor no gráfico) para os quais $f\left(\tilde{x}_{t+1}\right)$ dá resultados diferentes, violando o fato de $f$ ser uma função. Um mapa $x_{t+1}=f\left(x_{t}\right)$ periódico de menor período 4 pode exibir uma evolução temporal com uma das formas apresentadas na figura (b). . . . . . . . . . . . . 8

2.2 Mapa de retorno de Poincaré para um fluxo periódico. . . . . . . . . . 10

2.3 Diagrama teia de aranha exibindo os dez primeiros valores da seqüência produzida pelo mapa logístico $x_{t+1}=f_{\mu}\left(x_{t}\right)=\mu x_{t}\left(1-x_{t}\right)$, com $\mu=3.45$ e valor inicial $x_{0}=0.1$. O procedimento para obtenção da seqüência é o seguinte: a partir de um valor inicial $x_{0}$ no eixo horizontal (valores de $x_{t}$ ) é traçada uma reta vertical até interceptar o gráfico da função $f_{\mu}$. Obtemse, assim, o valor $x_{1}$ no eixo vertical (valores de $x_{t+1}$ ). Traça-se um linha horizontal nesse ponto até interceptar a diagonal $x_{t}=x_{t+1}$. Esse é o novo ponto a partir do qual é traçada uma nova linha vertical até interceptar o gráfico de $f_{\mu}$ para obter $x_{2}$. Repete-se o procedimento para os demais valores de $x_{t} \ldots \ldots \ldots \ldots \ldots \ldots \ldots \ldots \ldots$

2.4 Diagrama teia de aranha e diagrama de bifurcação para os mapas logístico (acima), da tenda (meio) e circular (abaixo). Esses mapas são parametrizados pelo parâmetro $a$, de acordo com as expressões (2.9), (2.10) e (2.11). O diagrama teia de aranha é construído para um determinado valor do parâmetro. O diagrama de bifurcação é obtido exibindo-se as seqüências assintóticas de valores $x_{t}$ para cada valor desse parâmetro (gráficos construídos através do web software de Michael Cross [Cross, 2003]) . . . . . . . 1 
2.5 Diferentes comportamentos da dinâmica do mapa logístico $x_{t+1}=f\left(x_{t}\right)=$ $\mu x_{t}\left(1-x_{t}\right)$ para diferentes valores de $\mu$. De cima para baixo, o mapa logístico exibe um ponto fixo atrator; órbitas de período 2 e 4; comportamento caótico para $\mu=3.9$; e comportamento intermitente com regiões de aparente periodicidade para $\mu=3.8282 \ldots \ldots \ldots \ldots$. . . . . . 14

2.6 Acima do diagrama de bifurcação, mostram-se diferentes dinâmicas do mapa logístico para alguns valores de $\mu$. Todos os gráficos foram obtidos a partir da condição inicial $x_{0}=0.55$. Note que a convergência das órbitas para um ponto fixo pode se dar de modo monótono ou oscilatório. A partir de $\mu>4$, os valores de $x_{t}$ tendem rapidamente para $-\infty \ldots \ldots$

2.7 As órbitas $x(t)=\sin (2 t)$ e $x(t)=\frac{1}{2} \sin (2 t)$ apresentam o mesmo período, $T=2$, e encontram-se em sincronismo de fase apesar de apresentarem amplitudes diferentes. . . . . . . . . . . . . . . 18

2.8 Pêndulos exibindo sincronismo em fase e anti-fase. . . . . . . . . . . . 19

2.9 Pêndulos. Em (a), os dois pêndulos exibem a mesma freqüência, mas não representam um exemplo de sincronismo: o sistema da forma que foi construído é, de fato, um único sistema que não pode ser decomposto. Em (b), dois sistemas que se encontram sem interação alguma podem exibir a mesma freqüência, mas também não representam um exemplo de sincronismo, pois não há interação entre eles. Finalmente, em (c), o apoio comum dos dois pêndulos não sendo perfeitamente rígido pode oferecer uma interação fraca entre eles e levá-los ao sincronismo. . . . . . . . . . . . 20

2.10 As duas figuras à esquerda são representações gráficas de acoplamento unidirecional (ou mestre-escravo); as duas figuras à direita, de acoplamento

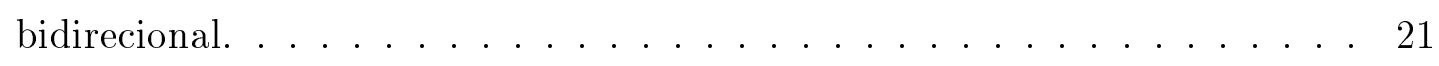

2.11 Modelo de acoplamento excitatório-inibitório de Wilson-Cowan. . . . . . . 22

2.12 Acoplamentos em uma rede unidimensional de mapas (1D). . . . . . . . 22 
2.13 Aqui são mostrados dois padrões de difusão. O primeiro é produzido por um CML conforme as equações (2.21) com $n=500, f(x)=3.99 x(1-x)$ e $\varepsilon=0.3$. Cada coluna apresenta os elementos da malha em um instante t. O tempo aumenta para a direita. Cada elemento tem o seu estado representado por um tom de cinza, de modo que a cor preto corresponde ao menor valor (zero, no caso). O segundo padrão é obtido através de um autômato celular através evolução da regra de 149 (numeração de Wolfram [Wolfram, 2001]). . . . . . . . . . . . . . . .

2.14 Esses diagramas representam duas formas de acoplamento bi-dimensional de mapas. Esses sistemas compartilham várias propriedades com automatos celulares e denominam-se as formas de acoplamento acima, a exemplo das vizinhanças de automatos celulares em duas dimensões, respectivamente, de acoplamentos em vizinhança de von Neumann e vizinhança de Moore [Gramß et al., 1998]. . . . . . . . . . . . . . . .

2.15 Padrões de difusão produzidos a partir de mapas logísticos acoplados em uma malha $2 D$ com acoplamentos em vizinhança de von Neumann, a partir de duas condições iniciais distintas. . . . . . . . . . . . . . .

2.16 Padrões de difusão produzidos a partir de mapas logíticos acoplados em uma malha $2 D$ com acoplamentos em vizinhança de Moore (à direita) e um padrão produzido a partir de equações de um sistema químico de reaçãodifusão retirado de [Bar-Yam, 1997] (à esquerda). . . . . . . . . . . . .

2.17 Transição de estados de um GCM de $N=50$ mapas logísticos. Transições de estados menos coerentes para um estado totalmente coerente são obtidas através do incremento da intensidade do acoplamento. O parâmetro $\mu=3.7$ fornece a não linearidade do sistema. Um estado totalmente dessincronizado, $k=N$, é obtido para um valor pequeno da constante de acoplamento, $\varepsilon=0.02$. Um estado coerente, com $k=1$, é obtido para o valor $\varepsilon=0.4$. Para os valores $\varepsilon=0.075$ e $\varepsilon=0.1$, o sistema exibe estados ordenados, com a formação de $k$ grupos de elementos sincronizados sendo $1<k<N$. O estado obtido para o valor $\varepsilon=0.075$ é, ainda, dito parcialmente ordenado [Kaneko \& Tsuda, 2000], exibindo "muitos" clusters com um "pequeno" número de elementos. . . . . . . . . . . . . . . 28 
3.1 Simetrias de reflexão e de rotação. A figura de cima destaca que o pentágono é invariante à reflexão sobre um eixo vertical, denominado eixo de reflexão. A figura de baixo destaca a simetria de rotação de ordem 5 de uma estrela, invariante por rotações de $2 \pi / 5$ em um ponto central. Note que ambas as formas - o pentágono e a estrela - exibem simultaneamente simetria de reflexão e de rotação. . . . . . . . . . . . . . . . . . . . . . .

3.2 Representação dos pixeis de uma imagem por elementos da rede de mapas e o acoplamento "local" que busca informações sobre a simetria da imagem. Assim, cada mapa está conectado a quatro vizinhos próximos e ao mapa diametralmento oposto. . . . . . . . . . . . . .

3.3 Figuras binárias (branco e preto) em matrizes $30 \times 30$ de pixeis. (a) A imagem da borboleta exibe simetria de reflexão com relação ao eixo das ordenadas, pois as órbitas $x_{i j}$ e $x_{i, m-j+1}$ sincronizam e os valores $y_{i}(t)$ e $z(t)$ tendem a zero. (b) A imagem do pato apresenta linhas não simétricas com relação à reflexão sobre o eixo das ordenadas, e os valores de $y_{i}(t)$ e $z(t)$ oscilam. . . . . . . . . . . . . . . . .

3.4 Mapa de bifurcação do mapa logístico. O valor do parâmetro de bifurcação do modelo é buscado a partir de valores do mapa isolado que apresentem o comportamento desejado para a rede. Nesse caso, buscam-se valores que levem o sistema a uma dinâmica periódica. . . . . . . . . . . . .

3.5 Diagramas estado-tempo, de uma rede de 50 mapas logísticos acoplados (GCM), apresentam a evolução dos estados de cada mapa. Uma escala de cinza é utilizada com os menores valores sendo representados por preto e os maiores valores por branco. Verifica-se, da esquerda para direita, que valores maiores do termo de acoplamento levam a um maior número de elementos sincronizados (colunas verticais semelhantes). Essa técnica de representação também pode ser encontrada em [Boccaletti et al., 2002] na análise de sistema estendidos. . . . . . . . . . . . . . . .

3.6 Matrizes de $8 \times 8$ pixeis. (a) Imagens em tons de cinza com diferentes níveis de assimetria vertical são corretamente classificadas. Quanto maior a assimetria, maior a amplitude da oscilação. (b) Diferentes imagens com adição de ruído $\eta(i j)$ a um número crescente de entradas (0,2.5, 10 e 20\%) são classificadas corretamente pelo modelo. O ruído adicionado tem média $E(\eta(i j))=0.005$ e variância $\sigma^{2}=10^{-5} \ldots \ldots \ldots \ldots$ 
3.7 A imagem inicial é modificada para um maior nível de assimetria nos instantes $t=40$ e $t=60$, que são tempos discretos correspondentes aos passos da simulação de (3.12). Nos instantes $t=80$ e $t=100$, a imagem é modificada para níveis de simetria maior até tornar-se totalmente simétrica no instante $t=120$. Esse comportamento se reflete nos valores de $z(t)$ que indicam as alterações na imagem a cada instante de tempo. . . . . . . . . . 43

3.8 Aplicação para centralização de eixo. . . . . . . . . . . . . . . . . . . 44

3.9 Centralização de eixo. O sistema é aplicado para detecção de simetria de reflexão com relação ao eixo vertical $V$ e ao eixo horizontal $H$ da matriz da imagem. Os sinais podem ser utilizados para se ajustar a posição da peça ao eixo (ver figura (3.8). As matrizes de pixeis são de $34 \times 34$. Os valores de entrada de cada pixel foram normalizados no intervalo [0 0.1$]$ e os parâmetros valem $\varepsilon=0.5$ e $\mu=3.2 \ldots \ldots \ldots$. . . . . . . .

3.10 Verificação de simetria com relação ao eixo vertical sobre fotos da atriz Nicole Kidman para teste do modelo. As imagens encontram-se digitalizadas em matrizes de pixeis de $70 \times 70$. Os valores de entrada de cada pixel foram normalizados no intervalo [0 0.0001] e os parâmetros valem $\varepsilon=0.5 \mathrm{e}$ $\mu=3.2$. As duas fotos mais abaixo foram manipuladas através de software para obtenção de imagens de rostos simétricos. Apenas no último a imagem é perfeitamente simétrica. . . . . . . . . . . . . . . . 4

4.1 Uma rede com 80 vértices representa algumas relações no comércio mundial. A rede não é regular, pois os nós apresentam diferentes quantidades de conexões (construída com o software Pajek [Pajek, 2003]). . . . . . . . .

4.2 Grafo $G=(V, A)$ não-direcionado com vértices $V=\{a, b, c, d\}$ e arestas $A=\{(a, b),(a, d),(a, c),(b, d)\} \ldots \ldots \ldots$

4.3 Distribuição do número de conexões (número de nós vizinhos) em redes regulares, aleatórias (redes small-world aqui incluídas) e sem escala. Modelos regulares apresentam uma distribuição uniforme de conexões. Em modelos aleatórios, o número de conexões segue uma distribuição de Poisson. Por último, em redes sem escala, o número de conexões obedece a uma distribuição de potências. . . . . . . . . . . . . . . 53

4.4 Rede regular e uma rede Watts-Strogatz (WS) produzida a partir dela. . . 57 
4.5 Valores de $C(p)$ e $L(p)$ para uma rede WS de 50 elementos e $k=4$, normalizados pelos valores do grafo regular, $C(0)$ e $L(0) \ldots \ldots$. . . . . 58

4.6 Rede regular e uma rede Newman-Watts (NW) produzida a partir dela. . . 59

4.7 Rede regular e uma rede "smallest-world" (SW) produzida a partir dela. . . 59

4.8 Diferentes modelos de redes. Dois modelos de conexões regulares: (a) uma rede de elementos totalmente conectados, esquema empregado no acoplamento de mapas do tipo GCM, com $N=16$ elementos; e (b) uma rede do tipo $k$-vizinhos mais próximos, empregado no acoplamento local de (CML), com $N=16$ e $k=4$. Três modelos de redes small-world construídos a partir do grafo regular (b): (c) Watts-Strogatz, WS; (d) Newman-Watts, NW; e (e) smallest-world, SW; ao final, uma rede aleatória (f) . . . . . . . . . . 60

4.9 Grafo não-direcionado e sua matriz de acoplamentos na tabela (4.2). . . . 62

4.10 Valores de $x_{i}(t)$, para mapas acoplados na forma do grafo da figura (4.9), sincronizam a partir de um certo instante $t=t_{0} \ldots \ldots \ldots$. . . . . 64

4.11 O menor caminho médio e o coeficiente de agregação normalizados pelos valores da rede regular, $L(p) / L(0)$ e $C(p) / C(0)$, para os diferentes modelos de redes small-world: (a) rede WS; (b) rede NW; e (c) rede SW. Resultados obtidos a partir de 20 realizações aleatórias de religações sobre uma rede do tipo $k$-vizinhos mais próximos, com $N=50$ e $k=4$. A probabilidade de religação é exibida em escala logarítmica. . . . . . . . . . . . 66

4.12 Diagramas de bifurcação para mapas acoplados em uma topologia de rede small-world WS (acima) e acoplados em uma topologia de rede regular (CML). Os dados foram obtidos para uma configuração de $N=50$ e $k=4$, computados após 2000 iterações, e exibidos os 50 últimos estados de cada sistema. Os parâmetros de acoplamento utilizados foram $\varepsilon=0.0001 \mathrm{e}$ $\varepsilon=0.03$. Observa-se que para valores maiores de $\varepsilon$, o sistema WS apresenta um maior número de clusters (ver detalhe na figura (4.13)), enquanto no sistema regular o número de clusters permanece inalterado. . . . . . . . . . 6

4.13 Detalhe da figura 4.12. Faixas largas indicam a presença de um maior número de clusters na rede WS. . . . . . . . . . . . . . . . 68

4.14 Diagrama de bifurcação a dois parâmetros destacando o papel do parâmetro $\varepsilon$ na formação de um maior número de clusters à medida que $\varepsilon$ cresce. . . . 
4.15 Mapas acoplados em diferentes topologias de redes small-world exibem diferentes números de clusters após o transiente da rede. As simulações foram realizadas partindo-se de uma rede regular de parâmetros $N=50$ e $k=4$. Para os parâmetros $\mu=3.45$ e $\varepsilon=0.0001$ a rede regular atinge um estado final com a formação de dois grupos de elementos - linhas pontilhadas no gráfico. A evolução do número de clusters é obtida através da matriz de distâncias de fase $D_{i j}\left(\tau_{\ell}\right)$, e exibe uma forte dependência com a

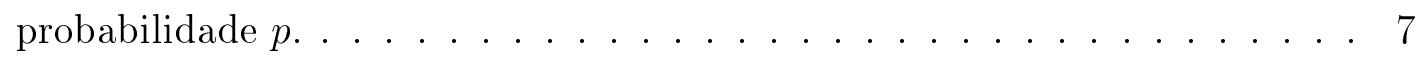

4.16 Evolução do número de clusters nos tempos $t=100,110$ e 400 para os diferentes tipos de rede WS, NW e SW, tendo sido empregados os valores de $p=$ $\{0,0.0003,0.0005,0.0008,0.001,0.0025,0.005,0.0075,0.01,0.025,0.05,0.075,0.1,0.2,0.4,0.6,0.8,1\}$. 71

5.1 Máquina de Turing determinista. . . . . . . . . . . . . . 74

5.2 Quaisquer circuitos de portas lógicas podem ser contruídos através de um arranjo, em princípio ilimitado, de neurônios de McCulloch-Pitts, reproduzindo as funções booleanas (AND, XOR, NOT). Acima, utilizam-se três elementos que implementam a função XOR, todos distintos e conectados em série, para solução do problema da paridade do conjunto de bits 1011. Cada operador XOR na figura (b) requer três neurônios que são representados na figura $($ a) por circunferências. . . . . . . . . . . . 78

5.3 A inclusão de recorrência adiciona memória ao sistema e permite a redução do número de elementos na execução de um procedimento computacional. Acima, emprega-se um único elemento que realiza a função XOR para solução do problema da paridade do conjunto de bits 1011. A figura (b) simplifica a representação de $($ a $) . \ldots \ldots \ldots \ldots$. . . . . . . . 79

5.4 Espaço invariante $S$ gerado para emulação de uma máquina de Turing com 3 símbolos e 3 estados. . . . . . . . . . . . . . . . . . . 83

5.5 Emulação de uma máquina de Turing através de mapas lineares em intervalos para verificação da paridade de bits das seqüências 1011 e 1010 . . . . 84

5.6 CML unidirecional. Cada elemento representa um mapa linear em $\mathbf{R}^{2}$. . 84

5.7 Representação da troca da dinâmica em sistemas de mapas com funções de troca. 
5.8 Mapas do padeiro em um sistema de funções de troca emulando uma máquina de Turing para verificação da paridade de bits. São exibidas seis seqüências diferentes de bits. À esquerda, encontram-se seqüências com quantidades ímpares de 1's; à direita seqüências com quantidades pares. Há a convergência para o valor correto, após algumas dezenas de iterações. 88

5.9 Os três primeiros estágios da construção da curva de Koch através do $L$ sistema (5.17). . . . . . . . . . . . . . . . . . 91

5.10 Curva de Koch produzida pelo sistema (5.20). . . . . . . . . . . . 93

5.11 Ilha de Koch original produzida pelo sistema (5.22) . . . . . . . . . 95

5.12 "Mutações" da ilha de Koch produzidas pelo sistema (5.23) . . . . . . . . 95

5.13 "Mutações" da ilha de Koch produzidas pelo sistema (5.24). . . . . . . . . 95

5.14 Forma obtida pelas transformações (5.25) sensíveis ao contexto . . . . . 96

5.15 Padrão "botânico" produzido pelo sistema de equações (5.26) . . . . . . . 97

5.16 Representação de um processo de difusão não uniforme em uma superfície. 97

5.17 (a) Padrão geométrico produzido por um sistema que emula o L-sistema: $\Sigma=\{F,+,-\}, \omega_{0}=F+F+F+F, p: F \mapsto F+F-F F+F+F-F$ e $\delta=\pi / 2$. (b) Um padrão geométrico dado por um sistema de mapas funções de troca. (c) Funções de troca permitem produzir um padrão que "mistura" os padrões das figuras (a) e (b) . . . . . . . . . . . . . . . 98

5.18 Padrões "botânicos"produzidos a partir de diferentes sistemas de mapas com funções de troca e que não possuem, de modo trivial, um L-sistema associado. . . . . . . . . . . . . . . . . . 99

5.19 Estrutura fractal de planta, produzido pelas transformações lineares da

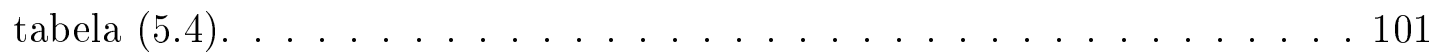

5.20 Estrutura fractal de planta, produzido pelas transformações lineares da tabela (5.5) . . . . . . . . . . . . . . . . . 101

5.21 Estrutura fractal de planta, produzida pela combinação das transformações lineares das tabelas $(5.4)$ e (5.5), com a função de troca (5.29) . . . . . . . 102

5.22 Triângulos de Sierpinski: mutações sucessivas por um sistema de mapas com funções de troca. . . . . . . . . . . . . . . . . . . . . . . 103 
6.1 "Nosso" ponto de vista: sistemas dinâmicos provendo processamento da informação por múltiplos elementos conectados. . . . . . . . . . . . 106

A.1 Exemplos de padrões gerados por automatos celulares, a partir de diferentes condições iniciais e diferentes regras $r$. Cada linha corresponde à configuração do autômato em um instante $t$ cujos estados evoluem verticalmente, de cima para baixo. . . . . . . . . . . . . . . . . . 115

A.2 Três exemplos de automatos celulares: a regra 90 que exibe um padrão semelhante ao dos fractais de Sierpinski [Brunde \& Havlin, 1995]; a regra 110 que possui capacidade de realizar computação universal [Wolfram, 2001]; e a regra 149 que exibe um padrão semelhante ao encontrado em algumas conchas de moluscos $[$ Wolfram, 2001] . . . . . . . . . . . . . 116

B.1 O triângulo de Sierpinski aparece como atrator do sistema de funções iteradas $F=\left\{f_{1}, f_{2}, f_{3}\right\}$ dado por (B.5) . . . . . . . . . . 119 


\section{Lista de Tabelas}

4.1 Valores de coeficientes de agregação, $C$, e menor caminho médio, $L$, medidos em algumas redes complexas. Os valores comparativos de $\ln (N)$ e $k / N$ estão próximos aos obtidos em modelos de redes aleatórias. Os dados das três primeiras colunas encontram-se compilados em (1) [Wang \& Chen, 2003] e (2) [Watts \& Strogatz, 1998] nos quais há maiores detalhes e referências sobre cada uma das medidas realizadas. . . . . . . . . . . . . . . . 54

4.2 Matriz de acoplamento $\mathbf{C}$ do grafo da figura $(4.9) \ldots \ldots \ldots 3$

4.3 Matriz para obtenção das distâncias entre nós do grafo (4.9) . . . . . . . 63

4.4 Matriz de distâncias de fases, em um instante $t<t_{0}$, exibe os seis elementos não-sincronizados. . . . . . . . . . . . . . . . . . 64

4.5 Matriz de distâncias de fases, em um instante $t>t_{0}$, exibe três clusters: $\{1,2,3\},\{4,5\}$ e $\{6\} \ldots \ldots \ldots \ldots \ldots \ldots \ldots \ldots \ldots \ldots \ldots \ldots \ldots \ldots \ldots \ldots$

5.1 Tuplas da máquina de Turing para um verificador de paridade. . . . . . . 75

5.2 Configurações da máquina de Turing para o verificador de paridade com entrada 1011. . . . . . . . . . . . . . . . . . . 76

5.3 Sucessivas cadeiais de símbolos produzidas pelo L-sistema (5.16). O número de elementos produzidos corresponde a seqüência de Fibonacci 1, 1, 2, 3, 5,

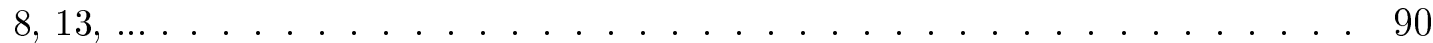

5.4 Sistema de transformações lineares (IFS) para a produção do padrão da figura (5.19) [Barnsley, 1988]. . . . . . . . . . . . . . . 100

5.5 Sistema de transformações lineares modificado que produz o padrão da

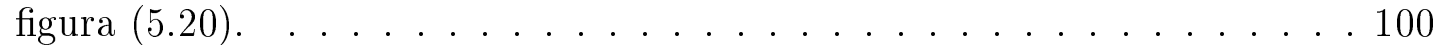

5.6 Sistema de transformações lineares modificado que produz o padrão da

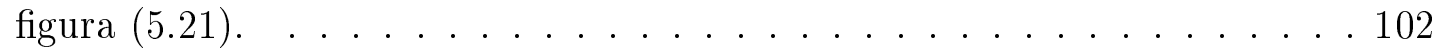


A.1 Regra de transição $\phi$. A seqüência da segunda linha, considerada como um número binário de 8 dígitos, fornece o número de Wolfram da regra. Acima, a regra 149. . . . . . . . . . . . . . . . . 115

A.2 Atualização dos estados do autômato celular pela regra 149 (tabela A.1). Aplica-se condição de contorno periódica (o primeiro elemento é vizinho do último). Destaca-se a vizinhança do quarto elemento e seu novo valor após a aplicação da regra de transição. . . . . . . . . . . . . . . . . . 115 


\section{Introdução}

Matemática e Física são dois conhecimentos teóricos da razão que devem determinar os seus objetos a priori.

Immanuel Kant

Neste trabalho, investiga-se a habilidade de sistemas compostos por mapas acoplados para solucionar três problemas, ou tarefas, em que normalmente são empregados sistemas computacionais. Tais problemas envolvem: processamento de imagens; auto-organização (formação de clusters); e processamento simbólico.

Sistemas dinâmicos parecem representar de modo mais adequado a forma como sistemas naturais, como o cérebro e sistemas sensoriais, processam e obtêm informações, explorando características menos presentes nos sistemas convencionais, baseados unicamente na lógica simbólica, como: paralelismo, tolerância a ruído e a falhas, etc. Entretanto, está longe de ter sido completamente compreendida a forma como esses sistemas operam. $\mathrm{O}$ estudo e a construção de novos modelos é, desse modo, importante na compreensão desses sistemas e da forma como operam. Empregamos aqui, sistemas de mapas acoplados para a construção desses novos modelos.

O uso de sistemas de mapas acoplados em tarefas que envolvem o processamento de imagens; auto-organização; ou o processamento de símbolos, já aparece em grande número de trabalhos ligados a neurociência, sistemas conexionistas e sistemas neurais. Para cada uma dessas tarefas, entretanto, desenvolvemos novos modelos e analisamos aspectos ainda não inteiramente explorados na abordagem desses problemas. Esses modelos, e a análise de suas características, constituem-se nas principais contribuições desta tese. 


\subsection{Detecção de simetria}

O processamento de imagens tem sido uma área de intensa aplicação de redes neurais. Normalmente, empregam-se redes em tempo discreto em tarefas de: reconhecimento de padrões [Kaneko, 1992], segmentação [Zhao, 2000b] e extração de contorno [Wei \& Ruan, 1999]. Entretanto, poucos modelos como esses parecem considerar a importante tarefa de extrair informações sobre a simetria de imagens. Um modelo de mapas acoplados teria a capacidade de extrair informações de simetria de imagens baseandose no sincronismo de seus elementos? Neste trabalho, construímos uma rede de mapas acoplados para detecção de simetrias de reflexão em imagens no plano. O modelo utiliza o acoplamento local e global dos mapas e emprega, motivado em modelos neurais, o sincronismo dos elementos da rede para extrair as informações de simetria das imagens. Uma série de simulações demonstram a capacidade do modelo no tratamento de imagens com ruído e de cenas dinâmicas.

\subsection{Auto-organização em redes não regulares}

Sistemas estendidos de mapas acoplados foram introduzidos a duas décadas por Kaneko [Kaneko, 1984] como modelos para o estudo de sistemas físicos espaço-temporais. Nesses sistemas, os mapas são acoplados de modo a formarem uma malha ou reticulado uniforme em que, cada elemento, conecta-se a seus elementos vizinhos. Em uma extensão importante desse modelo, todos elementos encontram-se conectados entre si. Esses sistemas podem exibir diferentes comportamentos assintóticos. Dentre eles, um estado ordenado, com a formação de grupos de elementos que sincronizam e passam a apresentar a mesma dinâmica [Kaneko \& Tsuda, 2000] e representa uma certa capacidade de auto-organização desses sistemas [Kaneko, 1994].

Modelos conexionistas e neurais, usualmente também empregam redes de elementos totalmente conectados [Hopfield, 1982] ou conectados em estruturas uniformes, sejam em camadas ou em uma malha de elementos competitivos. Entretanto, uma série de sistemas complexos, como redes sociais e redes neurais biológicas, a existência de uma malha regular de conexões ou a presençã de conexões entre todos os elementos parece pouco plausível. Muito recentemente, duas novas topologias, as redes sem escala [Barábasi, 2002] e as redes small-worlds [Watts, 1999], buscam melhor representar fenômenos complexos. Qual a capacidade de mapas acoplados nessas topologias, exibirem uma auto-organização 
semelhante a encontrada nos acoplamentos em forma de malhas regulares? Quais as diferenças dos estados obtidos? Demonstramos aqui, a capacidade de mapas acoplados em três diferentes topologias de small-worlds, de também exibirem a formação de estados ordenados. Nessas topologias empregam-se um número de conexões bastante próximo ao das malhas com acoplamento local mas com uma significativa redução da distância média entre os elementos da rede, constituindo-se na principal vantagem desses modelos. São realizadas simulações computacionais e comparados os resultados dos diferentes modelos.

\subsection{Sistemas programáveis e a produção de figuras e padrões no plano}

Por último, fazemos uma revisão das principais abordagens da capacidade de mapas exibirem computação universal, e empregamos um sistema de mapas com funções troca para emular e generalizar sistemas de produção (particularmente sistemas de produção de figuras e padrões geométricos no plano).

A computação universal de mapas, e sistemas constituídos de mapas, encontra diversas abordagens na literatura [Siegelmann \& Sontag, 1991], [Koiran et al., 1994], [Sato et al., 2000]. Verificamos a equivalência dessas abordagens na realização de um procedimento efetivo simples (um verificador de paridade de bits) e como um sistema de mapas com funções de troca fornece um modelo de computação a valores reais.

Empregamos, esse último modelo para construir um sistema que emula e estende sistemas de produção de figuras no plano, como L-sistemas [Prusinkiewicz \& Hanan, 1989] e sistemas de funções iteradas [Barnsley, 1988], e realizar processamento direto para obtenção de padrões fractais e de plantas. Em geral, sistemas de funções iteradas empregam um conjunto fixo de transformações lineares no plano. Aqui, utilizamos mapas mais gerais e funções de troca são empregadas para alterar a dinâmica do sistema segundo um contexto ou seu estado. O sistema obtido fornece uma fonte para a produção novos padrões biológicos e fractais. 


\subsection{Organização do trabalho}

O capítulo 2, trata de definições e conceitos básicos que são empregados ao longo do trabalho. As definições de mapa, dinâmica de uma função, sistemas estendidos, acoplamento e sincronismo, são particularmente importantes. Os demais capítulos tratam respectivamente de: detecção de simetria (capítulo 3); auto-organização em redes não regulares (capítulo 4); sistemas programáveis e a produção de figuras e padrões no plano (capítulo $5)$; conforme já foram descritos. Cada capítulo inicia-se com uma introdução ao problema específico de que trata e seus conceitos básicos, podendo, assim, serem lidos de modo independente uns dos outros. O capítulo sexto traz as conclusões deste estudo e aponta trabalhos futuros que podem serem realizados. Os anexos A e B apresentam uma breve introdução aos autômatos celulares e aos sistemas de funções iteradas.

\section{Observações sobre a notação empregada}

Em muitos termos desta tese como, small-worlds, clusters, coupled map lattices (CML) e globally coupled maps (GCM) opta-se pelo emprego do anglicismo, sendo esses, os termos normalmente empregados na literatura internacional. Os termos em língua estrangeira ocorrerão, entretanto, sempre em itálico. Também adota-se o "." (ponto) como separador decimal em números, apesar da norma culta exigir o uso da ","(vírgula). Isso permitiu facilitar a compatibilidade das figuras, produzidas por diferentes aplicativos em nossas simulações, e o texto. Não obstante a importância do uso correto das normas e da língua nas produções nacionais, espera-se não haver prejuízo ao entendimento do texto pelo emprego dessas notações. 


\section{Mapas, acoplamentos e sincronismo: conceitos preliminares}

O caos é uma ordem por decifrar. José Saramago

Neste capítulo, formulam-se definições relacionadas a mapas, sistemas acoplados e sincronismo utilizadas neste trabalho. São apresentadas simulações, por nós realizadas, que ilustram os conceitos abordados.

\subsection{Sistemas dinâmicos em tempo discreto}

Pode-se definir um sistema dinâmico discreto a partir de uma função que é composta, sucessivamente, com ela mesma [Holmgren, 1996]. Considere uma função $f$, tal que:

$$
\begin{aligned}
f^{0}(x) & =x, \\
f^{1}(x) & =f(x), \\
f^{2}(x) & =(f \circ f)(x), \\
f^{3}(x) & =(f \circ f \circ f)(x)=\left(f \circ f^{2}\right)(x), \\
f^{4}(x) & =(f \circ f \circ f \circ f)(x)=\left(f \circ f^{3}\right)(x), \\
\vdots & \\
f^{n}(x) & =\left(f \circ f^{n-1}\right)(x)
\end{aligned}
$$


sendo $n$ um número natural e $f^{n}=f \circ f^{n-1}$ a composição de $f \operatorname{com} f^{n-1}$. As expressões (2.1) representam as sucessivas aplicações de $f$ sobre o valor $x$, ou iterações.

Aqui, é suficiente considerar funções que levam valores reais a valores reais. Desse modo, define-se uma função $f: D \rightarrow C, C \subset \mathbf{R}^{n}$ e $D \subset \mathbf{R}^{m}$, como uma regra que associa a cada valor de $x \in D$ um único valor $f(x) \in C$ sendo, $D$ e $C$, respectivamente, o domínio e o contra-domínio de $f$. Em sistemas dinâmicos discretos, como em (2.1), o domínio e contra-domínio de $f$ satisfazem $D \subset C$, sendo, em geral, idênticos, de modo a permitir sucessivas aplicações de $f$.

Se cada iteração ocorre após um intervalo de tempo finito, as expressões (2.1) podem ser reescritas para descrever sistemas dinâmicos em tempo discreto como:

$$
\begin{aligned}
x_{0} & =f^{0}\left(x_{0}\right), \quad\left(=x_{0}\right) \\
x_{1} & =f^{1}\left(x_{0}\right)=f\left(x_{0}\right) \\
x_{2} & =f^{2}\left(x_{0}\right)=f\left(x_{1}\right), \\
x_{3} & =f^{3}\left(x_{0}\right)=f\left(x_{2}\right), \\
\vdots & \\
x_{t+1} & =f\left(x_{t}\right)
\end{aligned}
$$

Construída dessa forma, a equação de diferenças $x_{t+1}=f\left(x_{t}\right)$ caracteriza um sistema dinâmico em intervalos de tempo finito. $t$ é o índice temporal [Monteiro, 2002]. O comportamento dos pontos produzidos pelas sucessivas iterações de $f$ é chamado de dinâmica da função $f$ [Holmgren, 1996].

\section{Equações de diferenças}

Equações de diferenças da forma $x_{t+1}=f\left(x_{t}\right)$ são bastante particulares, já que são equações autônomas e de ordem 1.

Equações de diferenças autônomas são aquelas que não apresentam termos com dependência explícita do índice temporal. A seqüência de coeficientes binomiais de Pascal, por exemplo, é dada pela equação de diferenças não-autônoma $x_{k+1}=x_{k}+k$. 
A ordem de uma equação diferenças refere-se à dependência de $x_{t+1}$ dos termos anteriores da série $x_{t}, x_{t-1}, \ldots$ A diferença entre o maior e o menor índice temporal determina a ordem da equação. Desse modo, a seqüência de Fibonacci, dada pela equação de diferenças $x_{t}=x_{t-1}+x_{t-2}$, é de segunda ordem.

\subsection{Mapas, órbitas e pontos periódicos}

Um mapa é definido como um sistema dinâmico discreto da forma:

$$
x_{t+1}=f\left(x_{t}\right) \quad \text { ou } \quad x \mapsto f(x)
$$

sendo $f$ uma função em que o domínio e o contra-domínio coincidem. $t=0,1,2, \ldots$ é o índice associado aos sucessivos pontos da trajetória, ou órbita, formada pelo conjunto de pontos $\left\{x_{t}\right\}_{t>0}^{\infty}$ que satisfazem $(2.3)$.

\section{Órbitas}

Se $f$ é uma função tal que $f\left(x^{*}\right)=x^{*}$, então $x^{*}$ é um ponto fixo de $f$, isto é $f\left(x^{*}\right)=x^{*} \Rightarrow$ $f^{n}\left(x^{*}\right)=x^{*}$ para qualquer $n>0$. Um ponto $x^{*}$ é um ponto periódico de $f$ com período $k$ se $f^{k}\left(x^{*}\right)=x^{*}$. No caso de não haver valor $k_{0}<k$ tal que $f^{k_{0}}\left(x^{*}\right)=x^{*}$, diz-se que $x^{*}$ é um ponto periódico de $f$ com menor período $k$. Definições equivalentes são obtidas considerando-se as órbitas $\left\{x_{t}\right\}_{t>0}^{\infty}$ de mapas:

$$
\begin{array}{ll}
x^{*} \text { é ponto fixo de } f & \Longleftrightarrow x_{t}=x^{*} \text { para todo } t>0 \\
x^{*} \text { é ponto periódico de } f \text { com período } T & \Longleftrightarrow x_{t+T}^{*}=x_{t}^{*} \text { para todo } t>0
\end{array}
$$

As órbitas de um ponto periódico são denominadas de órbitas periódicas ou fechadas.

Finaliza-se esta seção com uma pequena proposição que aplica diversos dos conceitos apresentados e permite enfatizar propriedades que diferem mapas autônomos de ordem 1 de outras equações de diferenças.

Proposição. Considere $x_{t+1}=f\left(x_{t}\right)$ um mapa autônomo de ordem 1 e $x^{*}$ um ponto periódico de $f$ com menor período $T$. Então, não existe $x_{t_{0}}$ algum tal que $x_{t_{0}}=x_{t_{0}+k}$ com $0<k<T$. 

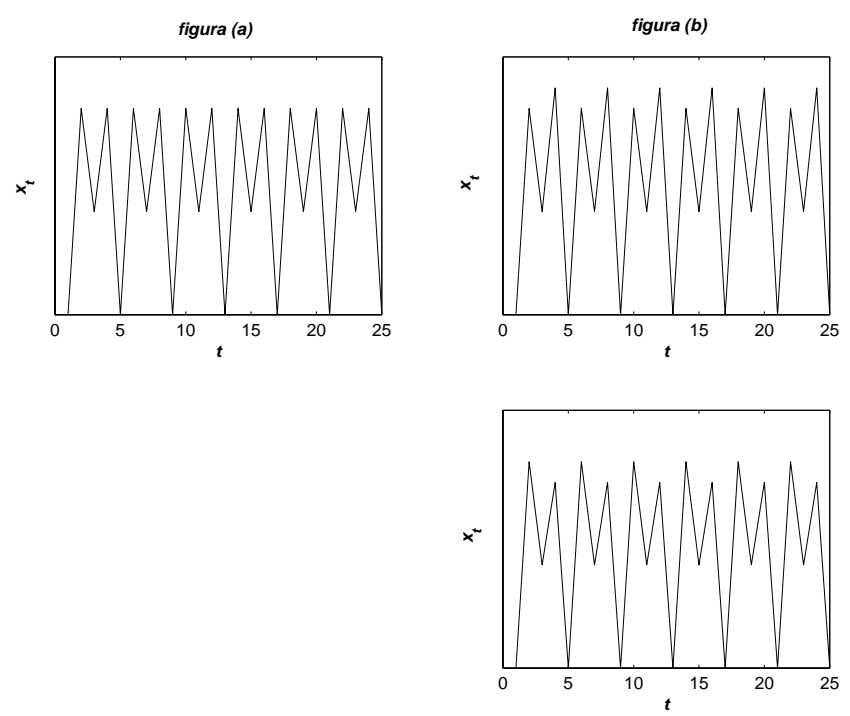

Figura 2.1: A seqüência mostrada na figura (a), uma órbita $\left\{x_{t}\right\}$ de período 4 , não pode ser obtida através de um mapa autônomo de ordem 1, pois apresenta pontos $\tilde{x}_{t}$ (os pontos de maior valor no gráfico) para os quais $f\left(\tilde{x}_{t+1}\right)$ dá resultados diferentes, violando o fato de $f$ ser uma função. Um mapa $x_{t+1}=f\left(x_{t}\right)$ periódico de menor período 4 pode exibir uma evolução temporal com uma das formas apresentadas na figura (b).

A proposição é imediata pelo fato de $f$ ser uma função e, portanto, não poder atribuir diferentes valores para $f\left(x^{*}\right)$.

Como conseqüência, observe que órbitas periódicas produzidas por mapas autônomos de ordem 1 não podem exibir um gráfico com a forma apresentada na figura (2.1)(a), devendo apresentar uma das formas que aparecem na figura (2.1)(b). Uma seqüência da figura (2.1)(a), entretanto, pode ser obtida por um mapa não-autônomo ou, ainda, por um mapa de ordem maior que 1. Fica claro também que se $x^{*}$ é ponto periódico com menor período $T$, a órbita $\left\{x_{t}\right\}_{t=0}^{\infty}$ de $x^{*}$ deve possuir $T$ pontos distintos.

Verificada a importância dessas definições para caracterizar mapas, trata-se a seguir da relação de mapas com sistemas dinâmicos em tempo contínuo.

\subsection{Mapas e campos vetoriais}

Existe uma conexão bastante grande entre sistemas dinâmicos em tempo discreto e campos vetoriais definidos por sistemas dinâmicos contínuos [Crawford, 1991]. Mais precisamente, podem-se obter sistemas dinâmicos discretos a partir de fluxos de sistemas contínuos. 
Considere o sistema autônomo de ordem $n$ definido por:

$$
\dot{x}=V(x), \quad x=x(t) \in \mathbf{R}^{n}, \quad t \in \mathbf{R}
$$

$V(x)$ identifica um campo vetorial em $\mathbf{R}^{n}$.

Dada uma condição inicial $x(0)$ existe um única solução $x(\tau)$ de (2.4) a qual define uma curva (fluxo) em $\mathbf{R}^{n}$ que é a trajetória de $x(0)$ ([Crawford, 1991]). Em outros termos, a associação $x(0) \rightarrow x(\tau)$ define:

$$
\phi_{\tau}: \mathbf{R}^{n} \rightarrow \mathbf{R}^{n}, \text { sendo } \phi_{\tau}(x(0))=x(\tau, x(0))
$$

o fluxo definido por (2.4).

Pode-se, então, considerar-se o mapa:

$$
x_{t+1}=f\left(x_{t}\right)
$$

sendo $x_{\tau}=f\left(x_{0}\right)$, e $f=\phi_{\tau}$ uma função a valores vetoriais [Guckenheimer \& Holmes, 1983] (pg. 16).

Dessa forma, um sistema dinâmico contínuo pode dar origem a um mapa discreto 1. Se $\phi_{t}$ é um fluxo liso (continuamente diferenciável), então $f$ é um difeomorfismo (um mapa liso e com inversa lisa) [Guckenheimer \& Holmes, 1983].

\section{Mapa de Poincaré}

O mapa de Poincaré (ou mapa de retorno de Poincaré) constitui-se numa outra forma de obter um mapa discreto a partir de um fluxo contínuo [Guckenheimer \& Holmes, 1983], [Monteiro, 2002]. Mapas podem ser mais simples de se analisar do que os sistemas de equações diferenciais que lhes dão origem e a seção de Poincaré é um dispositivo útil para reduzir fluxos contínuos em um espaço de dimensão $n$ a mapas em um espaço de dimensão $n-1$ [Ferrara \& Prado, 1994].

De um modo geral, um mapa de Poincaré é uma função $p$ que gera uma seqüência de pontos $x_{t}$ com que o fluxo $\phi: \mathbf{R}^{n} \rightarrow \mathbf{R}^{n}$ de um sistema contínuo intercepta um espaço

\footnotetext{
${ }^{1} \mathrm{O}$ fato de $f$ possuir inversa é uma conseqüência da forma que se obteve $f$ nesse exemplo. Pela definição de mapa, $f$ pode ser uma função mais geral que não admite inversa como, por exemplo, $x \mapsto a x^{2}$.
} 


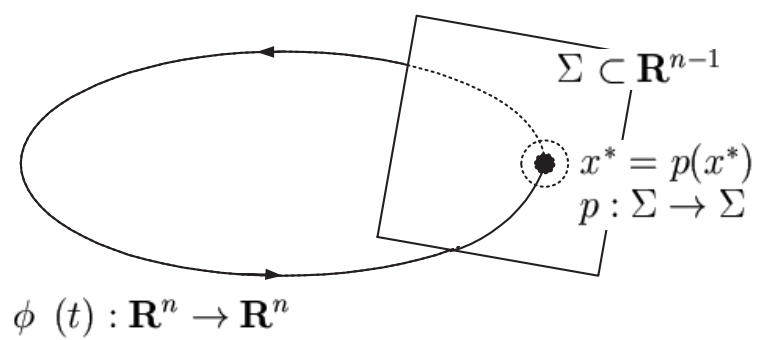

Figura 2.2: Mapa de retorno de Poincaré para um fluxo periódico.

transversal $\Sigma$ (seção de Poincaré) de dimensão $n-1$ [Monteiro, 2002]. Assim:

$$
x_{t+1}=p\left(x_{t}\right)
$$

Em particular, nesses mapas, procura-se pelos pontos fixos $x^{*}$, que são pontos tais que $x^{*}=p\left(x^{*}\right)$, uma vez que esses pontos determinam órbitas fechadas do fluxo $\phi . \mathrm{A}$ figura (2.2) procura ilustrar essa afirmação.

\subsection{Mapas parametrizados e bifurcações}

Considere mapas parametrizados da forma:

$$
x_{t+1}=f_{\mu}\left(x_{t}\right)
$$

sendo $\mu \in \mathbf{R}$ um parâmetro do mapa $f_{\mu}$.

A dinâmica da função $f_{\mu}$, isto é, os pontos produzidos pelas sucessivas iterações de $f_{\mu}$ [Holmgren, 1996], passa então a depender do valor do parâmetro $\mu$.

Alterações qualitativas na dinâmica da função $f_{\mu}$ são chamadas de bifurcações e os pontos em que essas mudanças de comportamento ocorrem denominam-se pontos de bifurcação. Por exemplo, os pontos produzidos pelas sucessivas iterações de $f_{\mu}$ podem levar a um ponto fixo ou uma seqüência periódica, de acordo com o valor de $\mu$.

\section{Diagramas de bifurcação}

Na análise qualitativa da dinâmica da função $f_{\mu}$, dois diagramas são bastante utilizados. Os diagramas teia de aranha (cobweb) facilitam a determinação dos pontos das sucessivas 


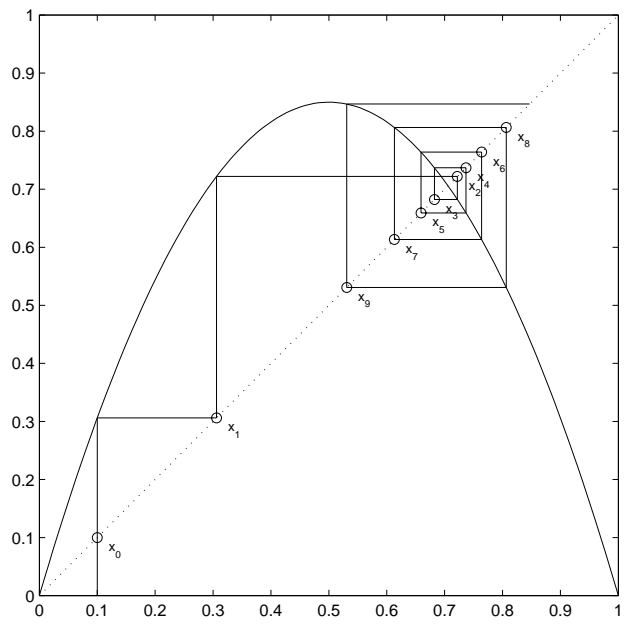

Figura 2.3: Diagrama teia de aranha exibindo os dez primeiros valores da seqüência produzida pelo mapa logístico $x_{t+1}=f_{\mu}\left(x_{t}\right)=\mu x_{t}\left(1-x_{t}\right)$, com $\mu=3.45$ e valor inicial $x_{0}=0.1$. O procedimento para obtenção da seqüência é o seguinte: a partir de um valor inicial $x_{0}$ no eixo horizontal (valores de $x_{t}$ ) é traçada uma reta vertical até interceptar o gráfico da função $f_{\mu}$. Obtem-se, assim, o valor $x_{1}$ no eixo vertical (valores de $x_{t+1}$ ). Traça-se um linha horizontal nesse ponto até interceptar a diagonal $x_{t}=x_{t+1}$. Esse é o novo ponto a partir do qual é traçada uma nova linha vertical até interceptar o gráfico de $f_{\mu}$ para obter $x_{2}$. Repete-se o procedimento para os demais valores de $x_{t}$.

iterações de um mapa [Monteiro, 2002] (ver figuras (2.3) e (2.4)). Os diagramas de bifurcação apresentam os pontos assintóticos das sucessivas iterações de $f_{\mu}$ para cada valor do parâmetro $\mu$ e possibilita identificar os valores de $\mu$ nos quais altera-se qualitativamente a dinâmica de $f_{\mu}$, que são os pontos de bifuracação.

Termina-se esta seção apresentando alguns exemplos importantes de mapas e alguns diagramas associados a esses mapas.

Mapas comumente empregados e que exibem dinâmicas complexas.

- Mapa logístico

$$
x_{t+1}=f\left(x_{t}\right)=a x_{t}\left(1-x_{t}\right)
$$

Alternativamente: $x_{t+1}=f\left(x_{t}\right)=1-a x_{t}^{2}$ [Kaneko \& Tsuda, 2000].

- Mapa da tenda (tent map)

$$
x_{t+1}=\left\{\begin{array}{cc}
a x_{t} & \text { se } 0 \leq x_{t} \leq 1 / a \\
a-a x_{t} & \text { se } 1 / a \leq x_{t} \leq 1
\end{array}\right.
$$

Alternativamente: $x_{t+1}=f\left(x_{t}\right)=a\left|\frac{1}{2}-\right| x_{t}-\frac{1}{2}||$ [Boffetta et al., 2002]. 
- Mapa circular

$$
x_{t+1}=f\left(x_{t}\right)=x_{t}+a+\frac{b}{2 \pi} \sin \left(2 \pi x_{t}\right)
$$

- Mapa deslocamento de Bernoulli (Bernoulli shift map)

$$
x_{t+1}=f\left(x_{t}\right)=2 x_{t}(\bmod 1)
$$

- Mapa cúbico

$$
x_{t+1}=f\left(x_{t}\right)=a x_{t}^{3}-a x_{t}+a \operatorname{com} x \in[-1,1]
$$

- Mapa de Hénon

$$
\left(x_{t+1}, y_{t+1}\right)=f\left(x_{t}, y_{t}\right)=\left(y_{t}+1-a x_{t}^{2}, b x_{t}\right)
$$

ou, usando uma notação alternativa:

$$
(x, y) \rightarrow\left(y+1-a x^{2}, b x\right)
$$

- Mapa do padeiro (baker's map) [Monteiro, 2002]

$$
\left(x_{t+1}, y_{t+1}\right)=\left\{\begin{array}{cc}
\left(2 x_{t}, a y_{t} / 2\right) & \text { se } 0 \leq x_{t} \leq 1 / 2 \\
\left(2 x_{t}-1, a y_{t} / 2+1 / 2\right) & \text { se } 1 / 2 \leq x_{t} \leq 1
\end{array}\right.
$$

\subsection{Dinâmica de uma função e caos}

A dinâmica da função $f_{\mu}$, ou o comportamento da seqüência de valores produzidos pelas sucessivas iterações de $f_{\mu}$, pode ser bastante diversa. Por exemplo, para diferentes valores de $\mu$, o mapa logístico $x_{t+1}=f\left(x_{t}\right)=\mu x_{t}\left(1-x_{t}\right)$ apresenta pontos fixos estáveis, órbitas de diferentes períodos ou ainda dinâmica caótica (ver figura (2.5)).

Definições rigorosas de dinâmica caótica podem ser encontradas em [Holmgren, 1996] e [Devaney, 1989] ${ }^{2}$. Neste trabalho, é suficiente uma definição da dinâmica caótica de uma função $f$ que considera apenas o comportamento exibido pelos pontos das sucessivas iterações de $f$ conforme descrito por [Kaplan \& Glass, 1995].

\section{Dinâmica caótica}

Segundo [Kaplan \& Glass, 1995] uma função possui caos ou dinâmica caótica se apresenta uma dinâmica aperiódica e limitada, em um sistema determinista, e exibe sensibilidade

\footnotetext{
${ }^{2}$ De fato, um sistema dinâmico $F$ é caótico se satisfaz três condições: a) pontos periódicos de $F$ são densos; b) $F$ é transitivo; c) $F$ depende sensivelmente das condições iniciais [Devaney, 1989].
} 

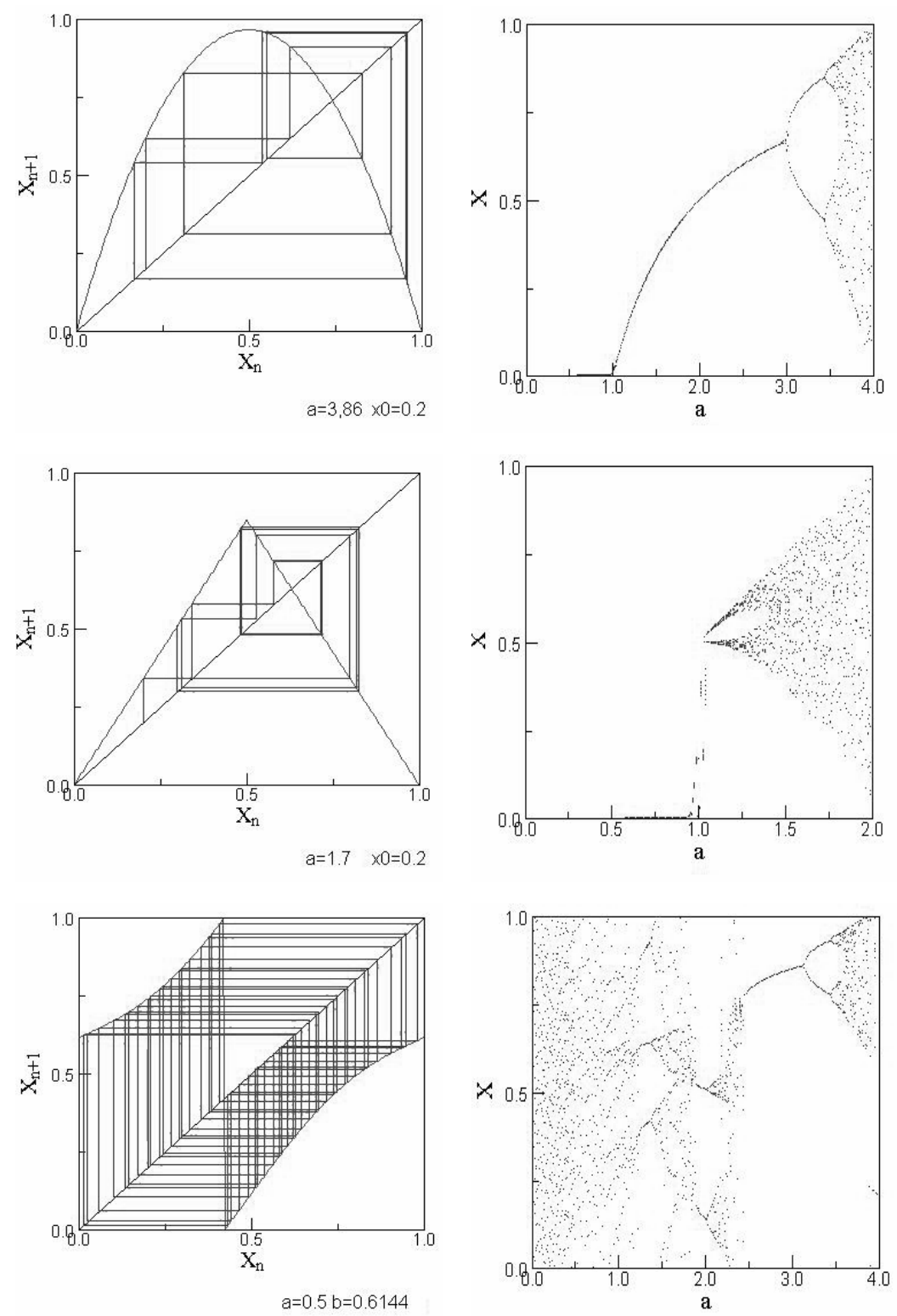

Figura 2.4: Diagrama teia de aranha e diagrama de bifurcação para os mapas logístico (acima), da tenda (meio) e circular (abaixo). Esses mapas são parametrizados pelo parâmetro $a$, de acordo com as expressões (2.9), (2.10) e (2.11). O diagrama teia de aranha é construído para um determinado valor do parâmetro. O diagrama de bifurcação é obtido exibindo-se as seqüências assintóticas de valores $x_{t}$ para cada valor desse parâmetro (gráficos construídos através do web software de Michael Cross [Cross, 2003]). 


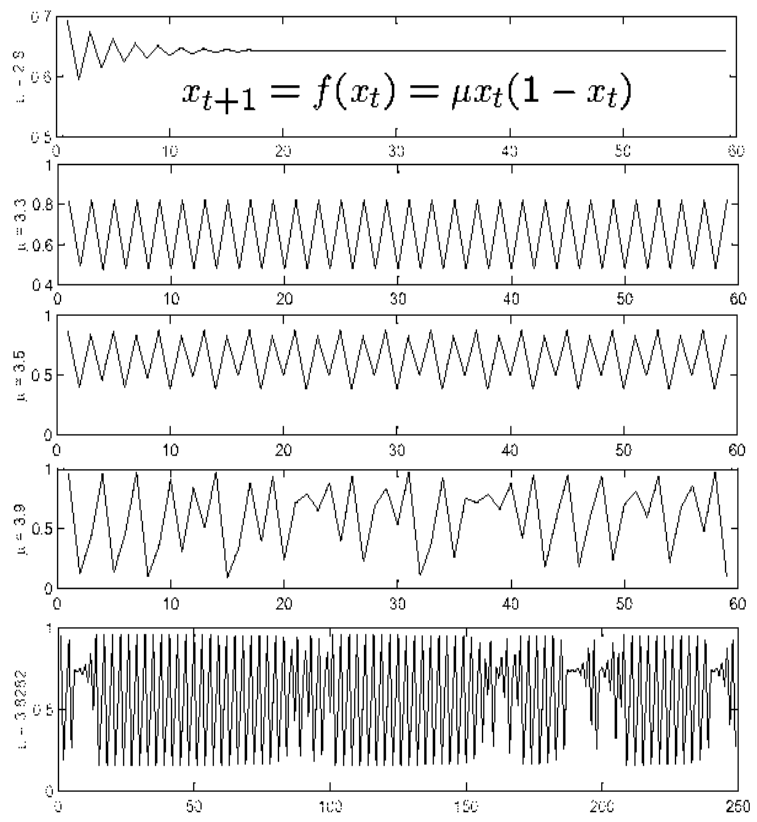

Figura 2.5: Diferentes comportamentos da dinâmica do mapa logístico $x_{t+1}=f\left(x_{t}\right)=$ $\mu x_{t}\left(1-x_{t}\right)$ para diferentes valores de $\mu$. De cima para baixo, o mapa logístico exibe um ponto fixo atrator; órbitas de período 2 e 4 ; comportamento caótico para $\mu=3.9$; e comportamento intermitente com regiões de aparente periodicidade para $\mu=3.8282$.

às condições iniciais. Cada um desses termos ganha uma definição mais precisa a seguir.

Aperiódico. Significa que nenhum ponto da órbita se repete.

Limitado. Significa que as sucessivas iterações de $f$ não levam os pontos da órbita a se aproximarem de $\pm \infty$.

Determinista. Significa que existe uma regra definida $f$ que governa a dinâmica e não possui termo aleatório.

Sensibilidade às condições iniciais. Significa que, dados quaisquer dois pontos iniciais próximos, as órbitas desses pontos se distanciam arbitrariamente ${ }^{3}$.

Na maior parte deste trabalho, interessa-se por órbitas que são periódicas ou que convergem para um ponto fixo.

As figuras (2.5) e (2.6) apresentam diferentes dinâmicas do mapa logístico, incluindo dinâmica caótica, para diferentes valores do parâmetro $\mu$.

\footnotetext{
${ }^{3}$ Formalmente $f: D \subset \mathbf{R}^{n} \rightarrow D \subset \mathbf{R}^{n}$ exibe sensibilidade às condições iniciais se existe $\delta>0$ tal que para qualquer $x \in D$ e qualquer $\varepsilon>0$, existe $y \in D$ e um número natural $n$ tal que $|x-y|<\varepsilon \mathrm{e}$ $\left|f^{n}(x)-f^{n}(y)\right|>\delta$ sendo $|$.$| a norma ou distância no espaço \mathbf{R}^{n}$ [Holmgren, 1996].
} 


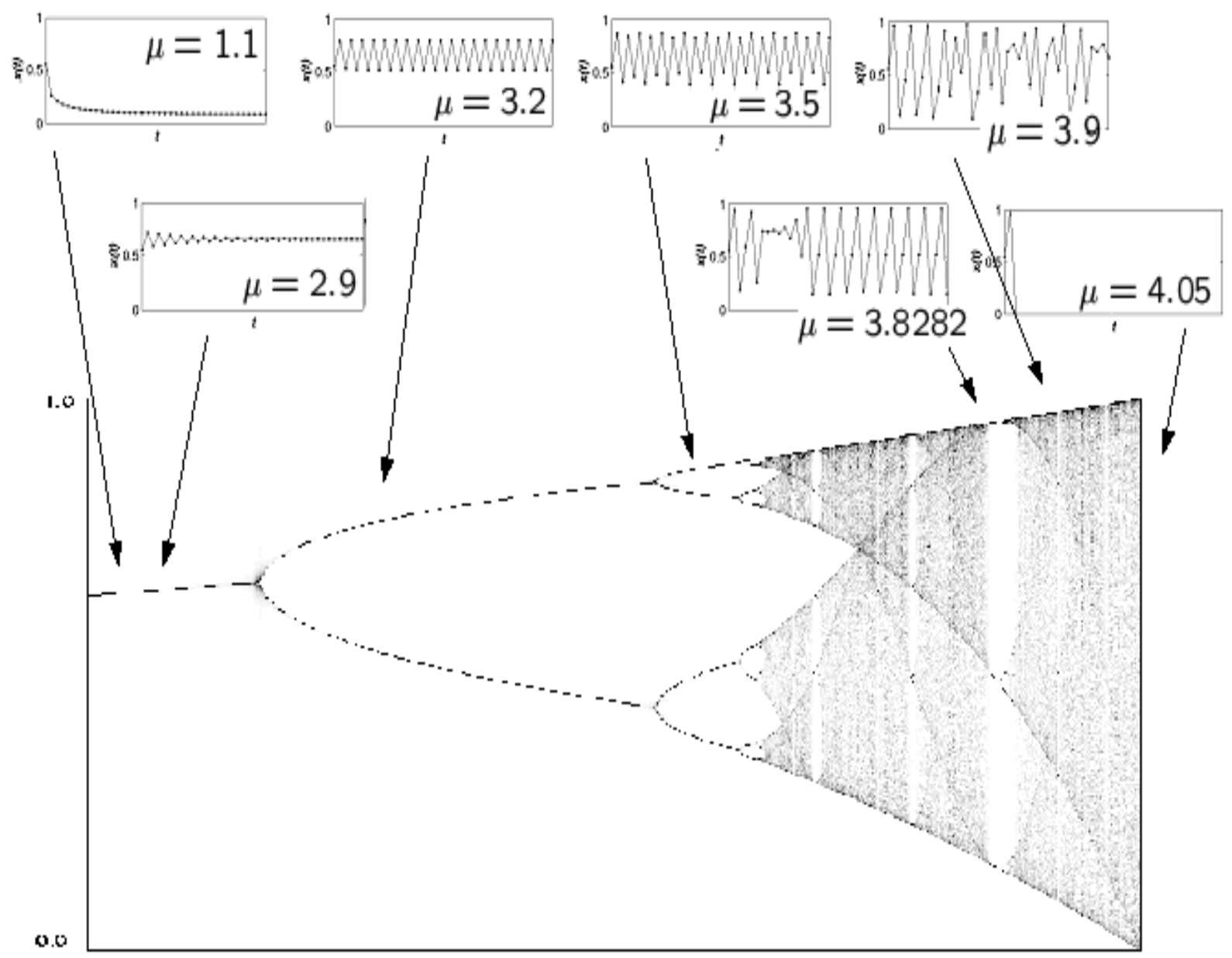

Figura 2.6: Acima do diagrama de bifurcação, mostram-se diferentes dinâmicas do mapa logístico para alguns valores de $\mu$. Todos os gráficos foram obtidos a partir da condição inicial $x_{0}=0.55$. Note que a convergência das órbitas para um ponto fixo pode se dar de modo monótono ou oscilatório. A partir de $\mu>4$, os valores de $x_{t}$ tendem rapidamente para $-\infty$. 
Uma série de outros aspectos podem ser considerados sobre mapas, desde o teorema da unicidade de soluções e o uso de diferentes normas ${ }^{4}$ na definição de sistemas dinâmicos, até a análise de autovalores, a classificação das bifurcações e teoremas de estabilidade. A dinâmica caótica costuma ser caracterizada pelo cálculo de constantes universais ${ }^{5}$, expoentes de Liapunov, entropia e dimensão fractal. Crê-se, entretanto, que os aspectos já abordados são suficientes para o que segue neste trabalho. Para os demais, consulte as referências [Guckenheimer \& Holmes, 1983], [Ferrara \& Prado, 1994], [Holmgren, 1996], [Kaplan \& Glass, 1995], [Monteiro, 2002].

Passa-se, então, a considerar sistemas constituídos de mapas que interagem e o comportamento de suas órbitas.

\subsection{Sincronismo e acoplamento}

A idéia de sincronização é um conceito bastante amplo e seu significado coloquial, em muitos casos, não se aplica, exigindo-se uma definição apropriada em cada contexto. Apresentam-se, então, algumas considerações antes de se introduzir uma definição de sincronismo de mapas.

Uma definição geral de sincronização pode ser encontrada em [Pikovsky et al., 2001]: um ajuste do ritmo entre objetos que oscilam, estando acoplados através de uma fraca interação entre eles. Cada um desses termos necessita de uma definição.

\section{Osciladores}

Objetos que oscilam. Consideram-se aqui objetos com oscilações auto-sustentadas, sendo a oscilação produzida, por exemplo, graças a uma fonte interna de energia do próprio sistema que compensa, ao menos em um intervalo em que se observam as oscilações, os efeitos dissipativos. Objetos como esses podem ser modelados por sistemas dinâmicos autônomos que apresentam comportamento oscilatório. Uma característica importante

\footnotetext{
${ }^{4}$ As normas desempenham um papel importante nos sistemas dinâmicos. Ao longo de todo este trabalho, considera-se a norma como a distância euclidiana estendida para o espaço $\mathbf{R}^{n}$, o que é suficiente aos nossos propósitos.

${ }^{5}$ Refere-se aqui às constantes de Feigenbaum.
} 
desses sistemas é que os estados oscilatórios são assintoticamente estáveis, isto é, o sistema evolui novamente segundo essa dinâmica após sofrer pequenas perturbações. Podese, então, considerar mapas que exibem uma dinâmica periódica ou caótica como esses objetos, sendo um oscilador ideal representado por um mapa periódico. 


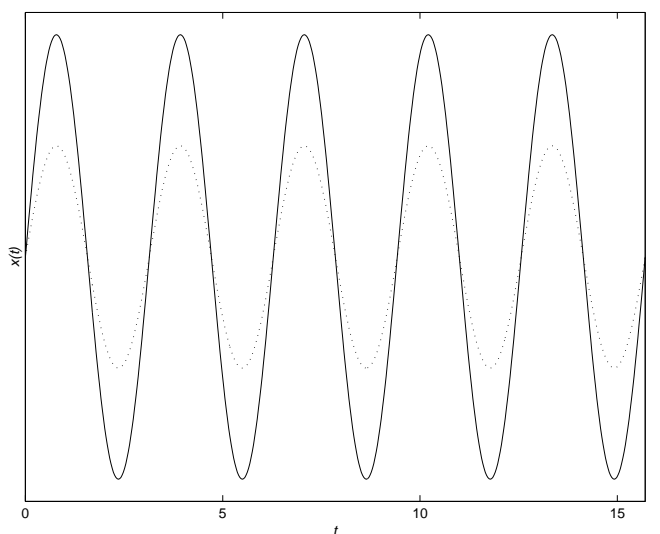

Figura 2.7: As órbitas $x(t)=\sin (2 t)$ e $x(t)=\frac{1}{2} \sin (2 t)$ apresentam o mesmo período, $T=$ 2, e encontram-se em sincronismo de fase apesar de apresentarem amplitudes diferentes.

O mapa logístico $x_{t+1}=\mu x_{t}\left(1-x_{t}\right)$ apresenta solução oscilatória em janelas do intervalo $3 \leq \mu<4$. Por exemplo, a solução com período $T=2$ ocorre para $3<\mu<$ $1+\sqrt{6}$ (ver figura (2.6)).

\section{Periodo e fase}

Seja uma órbita de pontos $x_{t}$ (contínua ou discreta). Ela é de período $T>0$ se $x_{t}=x_{t+T}$ para todo $t$. Em geral, $T$ refere-se ao menor período, e a idéia de ritmo está normalmente associada a esse período independentemente das amplitudes, ou das intensidades da oscilação (ver figura (2.7)).

Considere agora um campo vetorial sobre a circunferência $\dot{\theta}=f(\theta)$, em contraste ao campo vetorial sobre a reta $\dot{x}=f(x)$, sendo $\theta$ um ponto na circunferência. Esses sistemas fornecem os modelos mais básicos de sistemas que exibem oscilação [Strogatz, 1994]. Se $\theta$ varia uniformemente com o tempo, então $\dot{\theta}=\omega$, sendo $\omega$ uma constante, pois $\theta(t)=\omega t+\theta_{0}$ é a solução. Dado um valor inicial $\theta_{0}$, a projeção das órbitas $\theta(t)$, sobre os eixos horizontal e vertical, que são ortogonais e centrados na circunferência, possuem períodos $T=2 \pi / \omega$. Denomina-se $\theta(t)$ de fase e $\omega=2 \pi / T$ de freqüência angular da oscilação, que se diferencia da freqüência natural $\frac{1}{T}$ do sistema pela constante multiplicativa $2 \pi$.

Note que o raio da circunferência não altera a solução nem sua periodicidade. Podese, então, fornecer uma definição de sincronismo que independe da amplitude das oscilações produzidas. Diz-se que dois sistemas que oscilam apresentam sincronismo de fase [Pikovsky et al., 2001] se suas diferenças de fase $\theta_{2}-\theta_{1}$ mantêm-se limitadas, $\left|\theta_{2}-\theta_{1}\right| \leq$ 
(a)

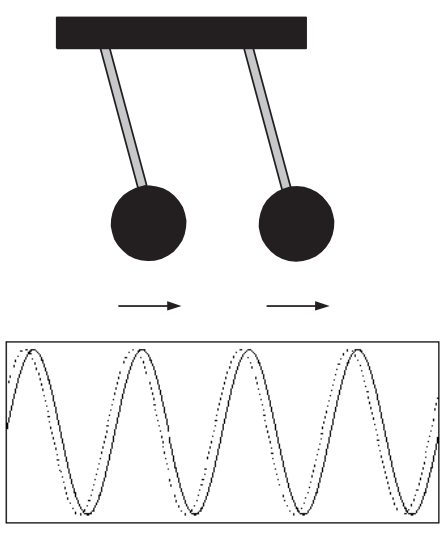

(b)

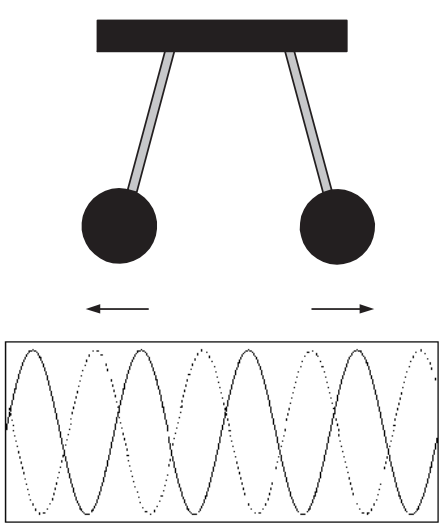

Figura 2.8: Pêndulos exibindo sincronismo em fase e anti-fase.

constante. A versão $\left|\theta_{2}-\theta_{1}\right|=$ constante é a mais freqüentemente empregada como sinônimo de sincronismo (ver figuras (2.7) e (2.8)).

Período e freqüência são quantidades bem definidas para mapas que exibem uma órbita periódica. Entretanto, dinâmicas caóticas são aperiódicas e, portanto, trazem uma série de dificuldades na aplicação direta desses conceitos.

\section{Sincronismo de mapas}

Uma forma mais restritiva de definir sincronismo é aquela que estabelece que dois sistemas encontram-se sincronizados se exibem órbitas iguais a medida que o tempo evolui. Nesse caso, diferentemente do sincronismo de fase, os sistemas apresentam não só período e freqüência idênticos, como também a mesma intensidade (amplitude). Sistemas que evoluem para essa forma de sincronismo apresentam sincronização completa, ou ainda idêntica ou convencional [Boccaletti et al., 2002]. Ao longo de todo este trabalho, considera-se essa forma de sincronização entre mapas que interagem. De modo formal, diz-se que dois mapas interagentes $a$ e $b$ sincronizam suas órbitas quando $\left|x_{t}^{a}-x_{t}^{b}\right| \rightarrow 0$ para $t \rightarrow \infty$. Esse tipo de definição pode ser aplicada para órbitas periódicas ou caóticas. 
(a)

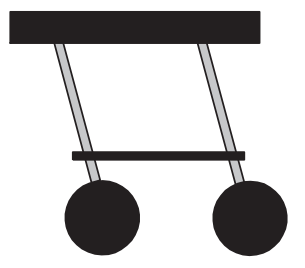

(b)

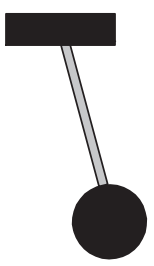

(c)

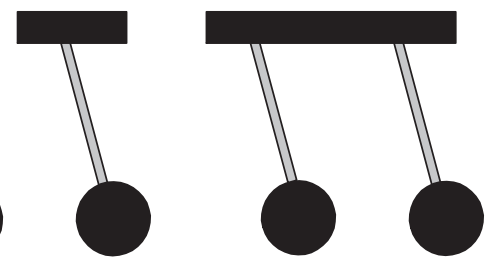

Figura 2.9: Pêndulos. Em (a), os dois pêndulos exibem a mesma freqüência, mas não representam um exemplo de sincronismo: o sistema da forma que foi construído é, de fato, um único sistema que não pode ser decomposto. Em (b), dois sistemas que se encontram sem interação alguma podem exibir a mesma freqüência, mas também não representam um exemplo de sincronismo, pois não há interação entre eles. Finalmente, em (c), o apoio comum dos dois pêndulos não sendo perfeitamente rígido pode oferecer uma interação fraca entre eles e levá-los ao sincronismo.

\section{Acoplamento}

Interação fraca. Para definir o que é uma interação fraca parte-se de dois contra-exemplos [Pikovsky et al., 2001]. Imagine dois pêndulos que possuem as hastes engatadas como na figura (2.9)(a). Pela rigidez do sistema, ou os dois pêndulos movem-se de modo sincronizado ou ambos encontram-se parados. Esse tipo de interação traz fortes limitações à dinâmica dos dois pêndulos, de forma que se pode considerar um único sistema que não pode ser decomposto. Essa não é, portanto, uma interação fraca. Suponha que os pêndulos estejam em locais distantes. Esses pêndulos também podem exibir movimento síncrono, mas alterações no movimento de um não interferem no movimento do outro. Nesse caso, há ausência de qualquer interação entre os sistemas (ver figura (2.9)(b)). Uma interação fraca, é portanto, no contexto de sincronização, uma interação que não inibe a individualidade de cada sistema e na qual a variação nas órbitas de um sistema faz com que as órbitas do outro sistema também sejam afetadas. Isso ocorre, por exemplo, no clássico experimento de dois pêndulos que possuem uma base de sustentação comum (figura $(2.9)(c))$. Fica claro, portanto, que a sincronização é um processo dinâmico.

Aqui, e no restante deste trabalho, considera-se um sistema acoplado aquele que apresenta um interação entre seus elementos, como definido acima, e que pode ser decomposto.

O mapa de Hénon dado por:

$$
(x, y) \rightarrow\left(y+1-a x^{2}, b x\right)
$$

não constitui um acoplamento de mapas, uma vez que a dinâmica da variável $y$, depende 


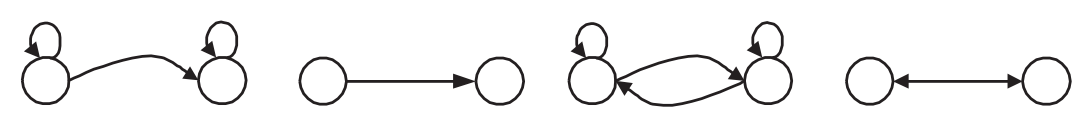

Figura 2.10: As duas figuras à esquerda são representações gráficas de acoplamento unidirecional (ou mestre-escravo); as duas figuras à direita, de acoplamento bidirecional.

totalmente da variável $x$ e não pode, portanto, ser decomposta.

Os mapas, a seguir, exibem um acoplamento simétrico excitatório-inibitório, isto é, a influência de um elemento sobre o outro possui mesma intensidade mas sinais opostos:

$$
\begin{aligned}
& x_{t+1}=(1-\varepsilon) x_{t}-\varepsilon y_{t} \\
& y_{t+1}=\varepsilon x_{t}+(1-\varepsilon) y_{t}
\end{aligned}
$$

sendo $0<\varepsilon<1$.

Distingüem-se duas formas de acoplamento [Boccaletti et al., 2002]. Quando a evolução de um dos sistemas acoplados é inalterada pelo acoplamento, a configuração resultante é chamada de acoplamento unidirecional ou acoplamento mestre-escravo (drive-response). Quando os dois sistemas influenciam-se, tem-se acoplamento bidirecional. Essas formas de acoplamento são esquematizadas na figura (2.10).

O sistema de mapas a seguir é uma rede neural artificial de dois neurônios acoplados [Wang, 1991]. O acoplamento se dá de forma semelhante ao proposto no modelo de tempo contínuo de Wilson-Cowan [Wilson \& Cowan, 1972] [Monteiro et al., 2002] como ilustrado na figura (2.11). Se $w_{12} w_{21}<0$ esse modelo é dito excitatório-inibitório, referindo-se à influência de cada unidade que forma o par:

$$
\begin{aligned}
& x_{t+1}=\sigma_{\mu}\left(w_{11} x_{t}+w_{12} y_{t}\right) \\
& y_{t+1}=\sigma_{\mu}\left(w_{21} x_{t}+w_{22} y_{t}\right)
\end{aligned}
$$

sendo $\sigma_{\mu}(z)=\frac{1}{1+e^{-\mu z}}$.

Aqui, é suficiente concluir dizendo que a forma como um sistema pode ser dividido define diferentes modos de acoplamento e dá origem a diferentes tipos de sincronismo. 


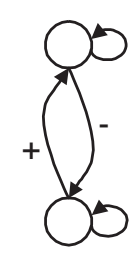

Figura 2.11: Modelo de acoplamento excitatório-inibitório de Wilson-Cowan.

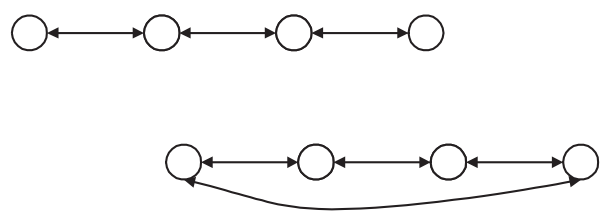

Figura 2.12: Acoplamentos em uma rede unidimensional de mapas (1D).

\subsection{Mapas acoplados e sistemas estendidos}

\section{Coupled map lattices}

Em sistemas acoplados formados por mais de 2 sub-sistemas, ou elementos, há várias possibilidades de acoplamento. As formas mais comumente utilizadas empregam acoplamentos regulares, isto é, acoplamentos em que a interação entre os diferentes elementos se dá de modo uniforme para todos os elementos do sistema, segundo uma regra.

Uma abordagem consiste em dispor os elementos em uma malha regular discreta (lattice), como mostrado na figura (2.12), na qual cada elemento interage com os seus vizinhos através da média dos estados desses vizinhos, conforme a equação:

$$
x_{i}(t+1)=(1-\varepsilon) f\left(x_{i}(t)\right)+\frac{\varepsilon}{2}\left\{f\left(x_{i+1}(t)\right)+f\left(x_{i-1}(t)\right)\right\}
$$

sendo $i=1, \ldots, N$ um elemento da malha de $N$ elementos. $x_{i}(t)$ são variáveis de estado calculadas no tempo discreto $t$. $f$ definida de $f: \mathbf{R} \rightarrow \mathbf{R}$ é, em geral, um mapa com dinâmica caótica, e $\varepsilon \in \mathbf{R}$ é a constante de acoplamento do sistema. Também, normalmente, adota-se a condição de contorno periódica $x_{N+1}=x_{1}$ (ver figura (2.12)), de modo que o último elemento da cadeia é considerado vizinho do primeiro. 


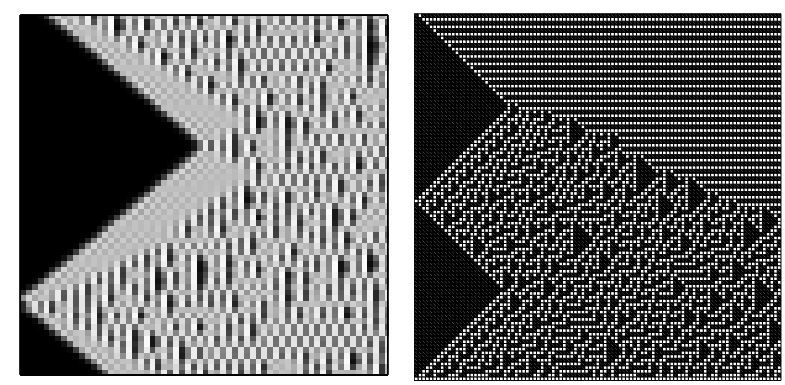

Figura 2.13: Aqui são mostrados dois padrões de difusão. O primeiro é produzido por um CML conforme as equações (2.21) com $n=500, f(x)=3.99 x(1-x)$ e $\varepsilon=0.3$. Cada coluna apresenta os elementos da malha em um instante $t$. O tempo aumenta para a direita. Cada elemento tem o seu estado representado por um tom de cinza, de modo que a cor preto corresponde ao menor valor (zero, no caso). O segundo padrão é obtido através de um autômato celular através evolução da regra de 149 (numeração de Wolfram [Wolfram, 2001]).

Esses sistemas denominados coupled map lattices (CML) foram introduzidos por Kaneko [Kaneko, 1984] como modelos para a análise de sistemas com dinâmica espaço-temporal caótica. Exemplos de tais sistemas envolvem mecânica de fluídos, sistemas químicos de reação-difusão, dinâmica de populações, formação de padrões e redes biológicas. Tais modelos são uma alternativa a sistemas de equações de derivadas parciais [Kaneko \& Tsuda, 2000], a exemplo também dos automatos celulares, com os quais eles compartilham grande número de propriedades [Wolfram, 2001] (ver figura (2.13) e anexo A).

Em variações desse modelo, estende-se o número de elementos vizinhos que interagem para os $k$-vizinhos mais próximos (ou seja, $k>2$ ) e modelos com acoplamento unidirecional [Kaneko \& Tsuda, 2000].

Os acoplamentos tratados até agora consideram os elementos distribuídos ao longo de uma linha. Malhas, ou redes, podem ser construídas considerando-se a distribuição dos elementos no espaço ( $2 D$ ou $3 D$, ou dimensões maiores). A figura (2.14) apresenta algumas possibilidades de acoplamento de elementos distribuídos em duas dimensões e as figuras (2.15) e (2.16) exibem padrões produzidos a partir dessas formas de acoplamento.

\section{Globally coupled maps}

Mapas globalmente acoplados (globally coupled maps, GCM) foram também introduzidos por Kaneko [Kaneko, 1992] como uma extensão dos CML. Esses também possuem acopla- 


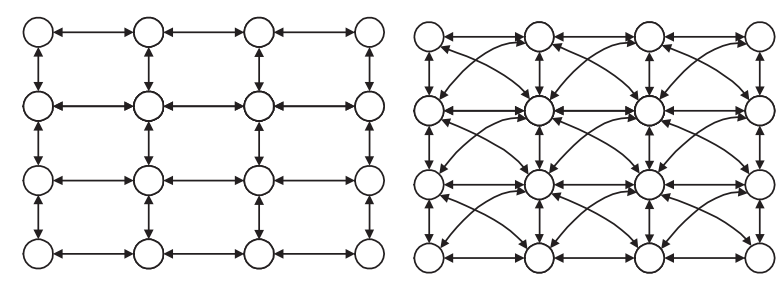

Figura 2.14: Esses diagramas representam duas formas de acoplamento bi-dimensional de mapas. Esses sistemas compartilham várias propriedades com automatos celulares e denominam-se as formas de acoplamento acima, a exemplo das vizinhanças de automatos celulares em duas dimensões, respectivamente, de acoplamentos em vizinhança de von Neumann e vizinhança de Moore [Gramß et al., 1998].

mento do tipo regular, com a mesma forma de acoplamento para todos os elementos da malha, no qual o estado de cada elemento recebe a influência da média dos estados de todos os demais, conforme a equação:

$$
x_{i}(t+1)=(1-\varepsilon) f\left(x_{i}(t)\right)+\frac{\varepsilon}{N} \sum_{j=1}^{N} f\left(x_{j}(t)\right)
$$

sendo, $i=1, \ldots, N$ um elemento da malha de $N$ elementos. $x_{i}(t)$ são as variáveis de estado calculadas no tempo discreto $t . f: \mathbf{R} \rightarrow \mathbf{R}$ é um mapa, em geral, com dinâmica caótica, e $\varepsilon \in \mathbf{R}$ a constante de acoplamento do sistema.

Adicionalmente, pode-se considerar mapas parametrizados de modo que o sistema (2.22) assume a forma:

$$
x_{i}(t+1)=(1-\varepsilon) f_{\mu}\left(x_{i}(t)\right)+\frac{\varepsilon}{N} \sum_{j=1}^{N} f_{\mu}\left(x_{j}(t)\right)
$$

sendo $\mu$ o parâmetro de bifurcação do mapa $f_{\mu}$.

Variações desse modelo podem incluir diferentes valores de acoplamento para cada elemento, ou seja, considerar $\varepsilon=\varepsilon_{i j}$, ou, ainda, a intensidade do acoplamento pode ser função do tempo $\varepsilon=\varepsilon_{i j}(t)$. 

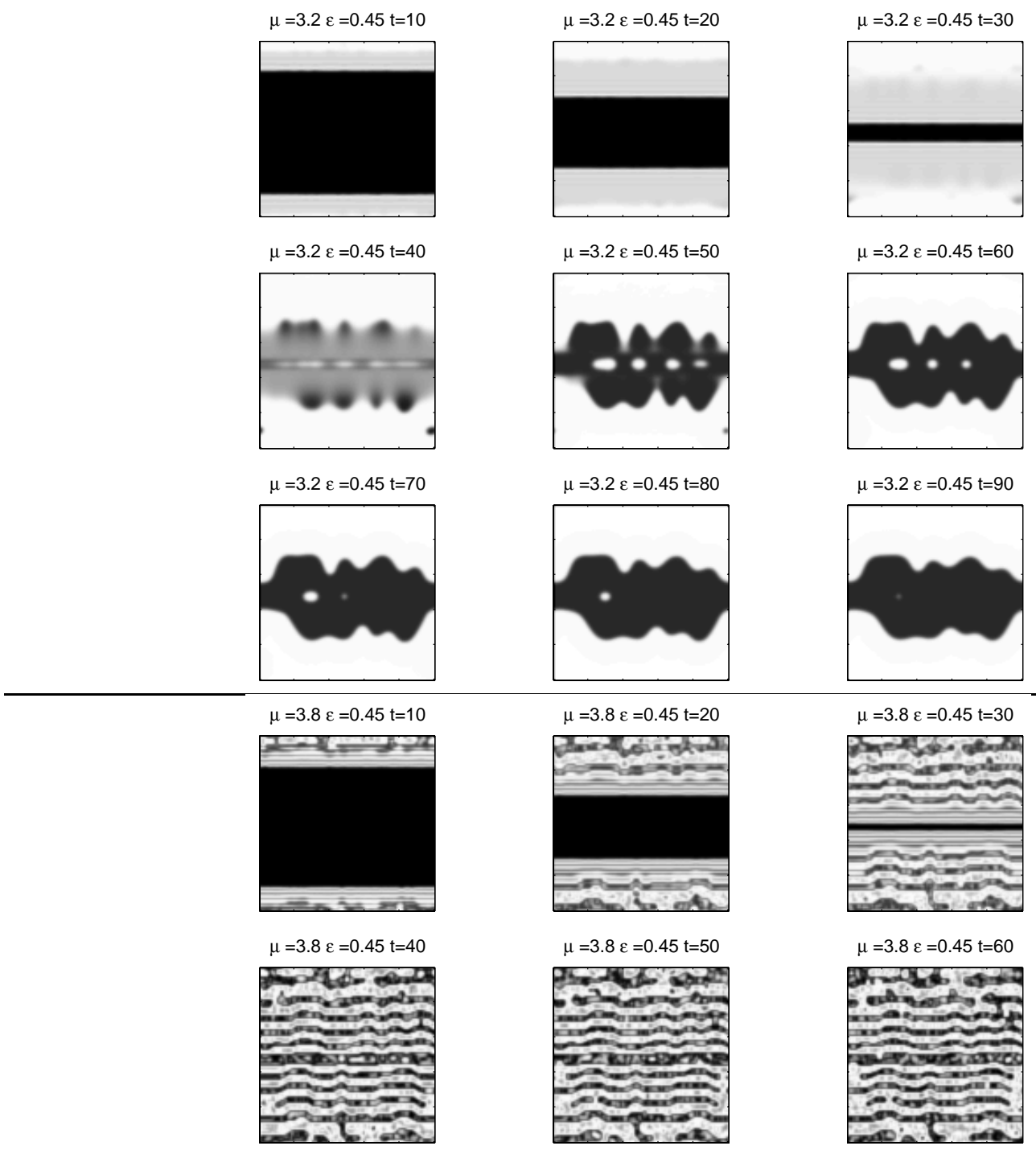

$\mu=3.8 \varepsilon=0.45 \mathrm{t}=60$

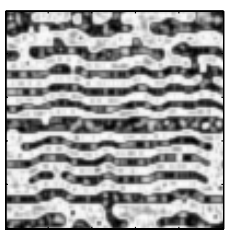

$\mu=3.8 \varepsilon=0.45 \mathrm{t}=70$
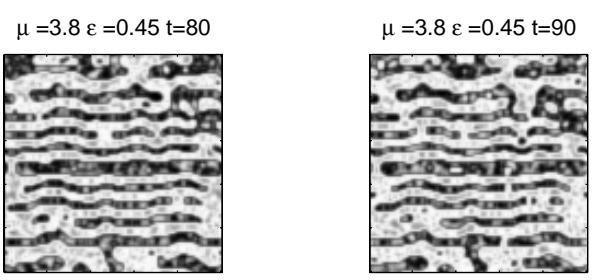

Figura 2.15: Padrões de difusão produzidos a partir de mapas logísticos acoplados em uma malha $2 D$ com acoplamentos em vizinhança de von Neumann, a partir de duas condições iniciais distintas. 

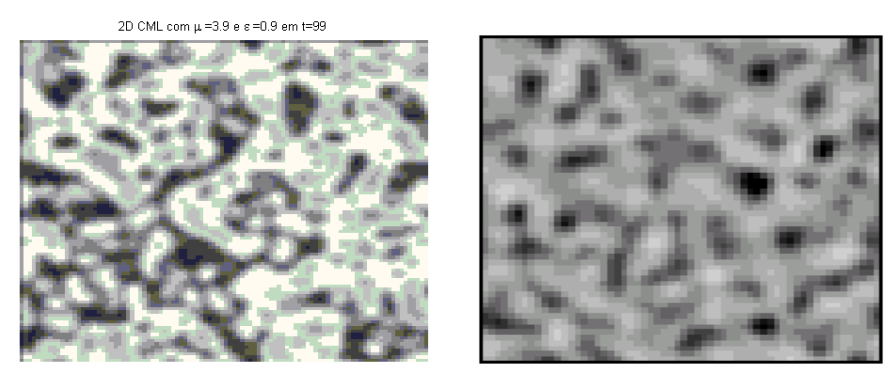

Figura 2.16: Padrões de difusão produzidos a partir de mapas logíticos acoplados em uma malha $2 D$ com acoplamentos em vizinhança de Moore (à direita) e um padrão produzido a partir de equações de um sistema químico de reação-difusão retirado de [Bar-Yam, 1997] (à esquerda).

\section{Clustering}

Tais sistemas exibem, basicamente, três tipos de comportamento assintótico, dependendo da intensidade do acoplamento e da não linearidade da dinâmica de $f_{\mu}$ governada pelo parâmetro $\mu$ [Kaneko \& Tsuda, 2000]:

- Um estado coerente, em que todos os elementos encontram-se completamente sincronizados, isto é $x_{i}=x_{j}{ }^{6}$ para quaisquer $i, j<N$.

- Um estado completamente dessincronizado, no qual nenhum elemento encontra-se sincronizado a outro.

- Um estado ordenado com a formação de clusters, cada cluster definido como um conjunto de elementos que se sincronizam, isto é: $x_{i}=x_{j}$ para todo $i, j$ pertencente ao mesmo cluster.

Seja $k$ o número de clusters e $N_{k}$ o número de elementos de cada cluster produzidos pela dinâmica de (2.22). Pode-se definir $k$ agrupamentos e reescrever a dinâmica (2.22) como segue [Boccaletti et al., 2002], [Popovych et al., 2002]:

\footnotetext{
${ }^{6}$ Considera-se, aqui, o comportamento assintótico do sistema e a notação $x_{i}=x_{j}$ significa que existe $t_{0}$ tal que $x_{i}(t)=x_{j}(t)$ para todo $t>t_{0}$.
} 


$$
\begin{array}{r}
x_{i_{1}}=x_{i_{2}}=\ldots=x_{i_{N_{1}}} \doteq X_{1} \\
x_{i_{N_{1}+1}}=x_{N_{1}+i_{2}}=\ldots=x_{N_{1}+N_{2}} \doteq X_{2} \\
\ldots \\
x_{i_{N_{1}+N_{2}+\ldots+1}}=\ldots=x_{N_{1}+N_{2}+\ldots+N_{k}} \doteq X_{k}
\end{array}
$$

e

$$
X_{i}(t+1)=(1-\varepsilon) f\left(X_{i}(t)\right)+\varepsilon \sum_{j=1}^{k} p_{j} f_{\mu}\left(X_{j}(t)\right)
$$

sendo $p_{j}=N_{j} / N$ a proporção de elementos no cluster $j$. Assim, (2.25) representa a dinâmica do cluster $i$.

O estado coerente corresponde a $k=1$ e o estado completamente dessincronizado a $k=N$. Os GCM costumam exibir transição de estados coerentes para completamente dessincronizados à medida que, se incrementa a não linearidade do sistema, ou se reduz a intensidade do acoplamento (ver figura (2.17)). Essa capacidade de organizar os elementos em grupos sincronizados pode ser vista como uma capacidade de auto-organização [Kaneko, 1994], [Kaneko \& Tsuda, 2000].

\section{Redes neurais artificiais}

Por fim, tem-se que sistemas de mapas acoplados, como os da equação (2.22), podem ser equivalentes a redes neurais recorrentes [Oliveira \& Monteiro, 2000]. De fato, fazendo-se $y_{i}(t)=f\left(x_{i}(t)\right)$ pode-se re-escrever (2.22) como:

$$
y_{i}(t+1)=f\left\{(1-\varepsilon) y_{i}(t)+\frac{\varepsilon}{N} \sum_{j=1}^{N} y_{j}(t)\right\}
$$

na qual escolhe-se $f$ como uma função do tipo sigmoidal (por exemplo $\tanh (\beta x)$ ) e termos de acoplamento $\varepsilon=\varepsilon_{i j}$, dependentes de cada elemento, obtidos por alguma regra de aprendizado, como a regra delta e os algoritmos de retropropagação dos modelos neurais [Haykin, 1999], [Kaneko \& Tsuda, 2000]. A expressão (2.26) é, então, uma rede neural recorrente. 

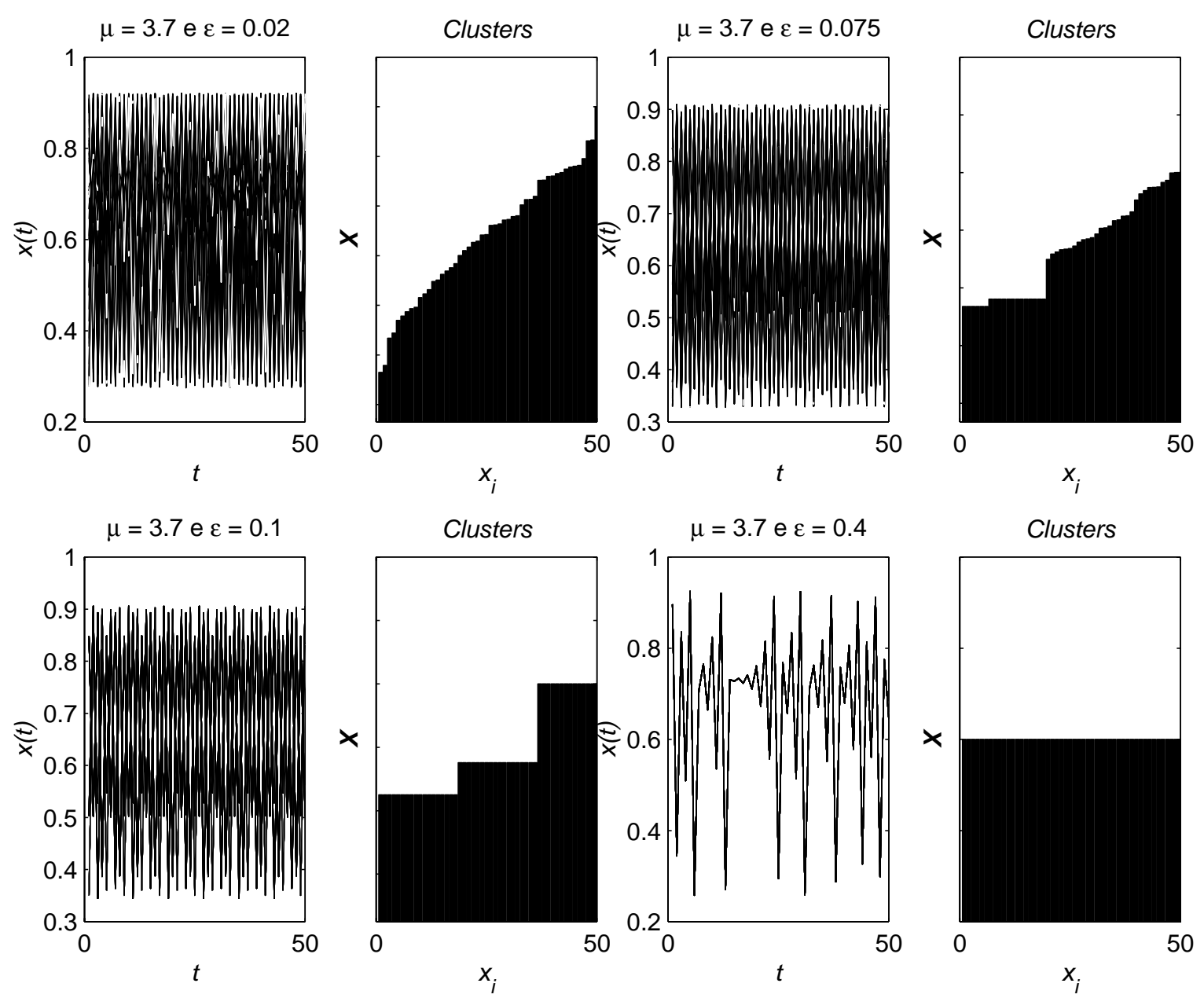

Figura 2.17: Transição de estados de um GCM de $N=50$ mapas logísticos. Transições de estados menos coerentes para um estado totalmente coerente são obtidas através do incremento da intensidade do acoplamento. O parâmetro $\mu=3.7$ fornece a não linearidade do sistema. Um estado totalmente dessincronizado, $k=N$, é obtido para um valor pequeno da constante de acoplamento, $\varepsilon=0.02$. Um estado coerente, com $k=1$, é obtido para o valor $\varepsilon=0.4$. Para os valores $\varepsilon=0.075$ e $\varepsilon=0.1$, o sistema exibe estados ordenados, com a formação de $k$ grupos de elementos sincronizados sendo $1<k<N$. O estado obtido para o valor $\varepsilon=0.075$ é, ainda, dito parcialmente ordenado [Kaneko \& Tsuda, 2000], exibindo "muitos" clusters com um "pequeno" número de elementos. 


\subsection{Conclusão e sumário}

Apresentamos, neste capítulo, alguns conceitos relacionados a mapas, definidos em termos de sistemas dinâmicos em tempo discreto. Esses sistemas exibem uma diversidade de comportamentos, incluindo dinâmica caótica, e apresentam relações com sistemas em tempo contínuo. Esses mapas podem ser organizados de forma a interagirem, no sentido de que a dinâmica de um passa a influenciar a dinâmica do outro. Denomina-se essa interação de acoplamento. Sistemas com um grande número de mapas acoplados são chamados de sistemas estendidos. Os modelos estendidos mais freqüentemente empregados adotam geometrias regulares de acoplamento, em que todos os elemento apresentam o mesmo padrão de conexão. Os modelos mais importantes são os que introduzem acoplamentos com a média dos estados de vizinhos mais próximos (CML, coupled map lattices) ou com a média do estado global da rede (GCM, globally coupled maps). Esses modelos são empregados no estudo de sistemas com dinâmica espaço-temporal (fluídos, populações etc.) assim como são usados os automatos celulares. Dentre as propriedades mais interessantes, encontramos a possibilidade de suas dinâmicas evoluirem para a formação de clusters, grupos de elementos que exibem a mesma dinâmica de modo síncrono, e associamos a esse comportamento assintótico uma capacidade de auto-organização desses sistemas. Desse modo, abordamos os principais aspectos que servem de base para o restante deste trabalho. 


\section{Processamento de imagens: detecção de simetria}

Symmetry is immunity to a possible change. Joseph Rosen

Este capítulo é uma extensão dos trabalhos anteriores de [Oliveira \& Monteiro, 2000] e [Oliveira \& Monteiro, 2002a]. Aqui, investiga-se o uso de mapas acoplados para a detecção de simetria de reflexão de imagens. A detecção dessa propriedade, tem um papel importante na análise de imagens, como na redução de tarefas de detecção de padrões e de reconhecimento, como as desempenhadas pelo cérebro. Embora diversos métodos possam ser empregados, como a simples comparação entre pixeis, interessa-nos aqui a construção de um modelo capaz de extrair informações da imagem através da dinâmica de seus elementos, aproximando-se, desse modo, da forma de funcionamento que alguns sistemas biológicos parecem apresentar.

\subsection{Simetria}

Embora seja um conceito matemático sofisticado, a idéia de simetria é mais freqüentemente associada a imagens no plano. Sua formalização se dá via teoria de grupos, tendo aplicações em diversos campos da ciência, como na física, na teoria da informação e no estudo de sistemas dinâmicos. De modo geral, simetria é a possibilidade de efetuar uma mudança, mantendo certos aspectos inalterados [Rosen, 1995]. Tem-se, então, simetria com relação a esses aspectos e essa definição permite identificar simetrias que vão além de seu significado coloquial. As equações de Lorenz [Lorenz, 1963] fornecem um exemplo da aplicação desse conceito. Tais equações são escritas como: 


$$
\begin{array}{r}
\dot{x}=\sigma(y-x) \\
\dot{y}=r x-y-x z \\
\dot{z}=x y-b z
\end{array}
$$

$\sigma, r, b>0$ são parâmetros e o ponto significa derivada temporal. As soluções desse sistema são simétricas. Se for efetuada a troca de variáveis $(x, y) \rightarrow(-x,-y)$ em (3.1) (nossa possibilidade de mudança), as equações mantêm-se inalteradas sendo, portanto, simétricas nesse aspecto. Em outros termos, se $(x(t), y(t), z(t))$ é uma solução de (3.1), então $(-x(t),-y(t), z(t))$ também é [Strogatz, 1994].

\section{Simetrias de reflexão e de rotação}

Para nossos propósitos, é suficiente considerar os aspectos geométricos de simetrias no plano.

Simetria é uma das propriedades básicas de formas, imagens e objetos, desempenhando um papel importante na visão computacional, especialmente, em tarefas como reconhecimento, busca, inspeção e extração de outras propriedades dos objetos. Transformações no plano $\left(\mathbf{R}^{2}\right)$ permitem identificar, ao menos, dois tipos de simetria ${ }^{1}$ :

- Simetrias de reflexão. Uma figura no plano apresenta simetria de reflexão se ela é invariante a reflexões com respeito a uma ou mais linhas, denominadas eixos de reflexão.

- Simetrias de rotação. Uma figura no plano apresenta simetria de rotação de ordem $K$ se é invariante a rotações de $2 \pi / K$ radianos em torno de um ponto central da figura e $K$ é o maior inteiro.

Essas simetrias encontram-se ilustradas na figura (3.1). As transformações $T: \mathbf{R}^{2} \rightarrow$ $\mathbf{R}^{2}$ de reflexão sobre uma linha $r$ no plano e as de rotação em torno um ponto $c$ são

\footnotetext{
${ }^{1}$ Mais precisamente e conforme a definição usada: simetria é a propriedade de uma configuração $\mathbf{X}$ no plano permanecer invariante em relação a essas transformações $T$, isto é, $T(\mathbf{X})=\mathbf{X}$.
} 


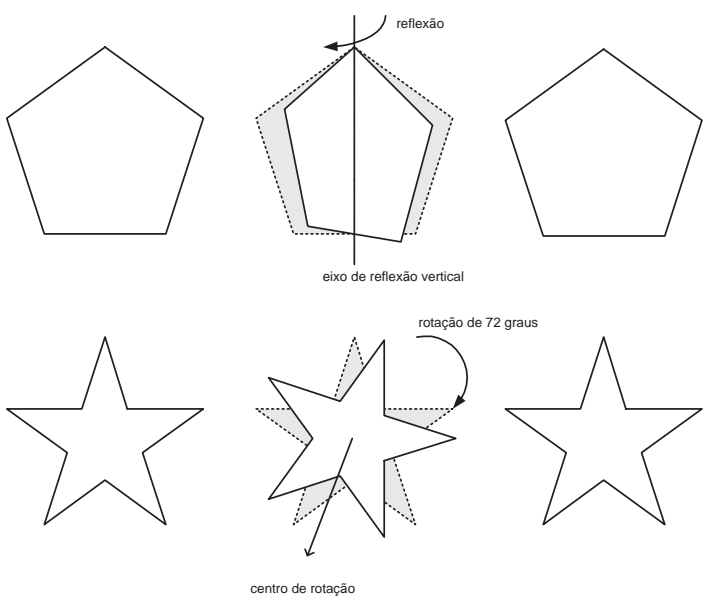

Figura 3.1: Simetrias de reflexão e de rotação. A figura de cima destaca que o pentágono é invariante à reflexão sobre um eixo vertical, denominado eixo de reflexão. A figura de baixo destaca a simetria de rotação de ordem 5 de uma estrela, invariante por rotações de $2 \pi / 5$ em um ponto central. Note que ambas as formas - o pentágono e a estrela - exibem simultaneamente simetria de reflexão e de rotação.

mais facilmente definidas considerando-se uma mudança de coordenadas de modo que $r=(0, y)$ é o eixo vertical do plano e $c=(0,0)$. Desse modo, escreve-se:

$$
T_{(r)}(x, y)=(-x, y)
$$

a reflexão de $(x, y)$ sobre $r=(0, y)$ e,

$$
T_{(\alpha, c)}(x, y)=(x \cos \alpha-y \sin \alpha, x \sin \alpha+y \cos \alpha)
$$

a rotação de $(x, y)$ de $\alpha$ radianos em torno de $c=(0,0)$.

\section{Detecção de simetria}

Simetria pode ser considerada uma propriedade binária das figuras no plano, isto é, uma figura é ou não simétrica. Interessa, aqui, identificar, dado um eixo de reflexão, se uma imagem apresenta ou não simetria de reflexão com respeito a esse eixo. Existem vários métodos para detecção de simetria, e uma revisão de técnicas pode ser encontrada em [Shen et al., 1999]. Poucos métodos baseiam-se em modelos de redes neurais artificiais [Shen et al., 1999]. Duas exceções são encontradas em [Sejnowski et al., 1986] e 
[Konen \& Malsburg, 1993] em que são adotadas, respectivamente, técnicas supervisionadas e não supervisionadas de aprendizado. A razão desse pouco uso de modelos neurais reside no fato de que, em geral, a detecção de simetria é um problema combinatorial em que a complexidade cresce exponencialmente com o número de elementos da imagem. Essa complexidade, sugere a estratégia de inserirmos na arquitetura da rede uma estrutura específica para o problema em questão, tornando-a menos ampla que um modelo geral para a percepção de imagens. O problema, então, seria o de buscar um modelo específico para a detecção capaz de apresentar um certo grau de generalidade.

Aqui, nossa escolha cai no uso de um modelo geral de mapas acoplados, tendo em conta a capacidade desses sistemas de representarem padrões espaciais no tempo, adequando-os à solução do nosso problema de detecção de simetria.

\section{Sistemas acoplados e imagens}

Sistemas de elementos acoplados têm sido utilizados na solução de uma larga classe de problemas: reconhecimento [Kaneko, 1992], segmentação de imagens [Zhao et al., 2000], [Zhao, 2000b], [Zhao \& Macau, 2001], [Chen \& Wang, 2002], [Hayashi, 1994], formação de padrões [Nishii, 1998], memórias associativas [He et al., 1999] e detecção de contorno [Wei \& Ruan, 1999]. A maior parte desses trabalhos baseia-se no sincronismo de elementos do sistema, a partir do qual se retira a informação procurada.

Sistemas acoplados, mais especificamente, mapas acoplados, assim como modelos neurais recorrentes baseados em funções do tipo sigmóide (ver equivalência entre esses modelos na seção 2.7), permitem representar relações espaciais entre os elementos da rede [Kaneko, 1992], [Zhao et al., 2000]. Isso significa que o estado da rede, em qualquer momento, depende da relação espacial de seus elementos. Essa propriedade pode ser utilizada para se inserir, em nosso modelo, relações de simetria. Dessa forma, esses sistemas fornecem uma classe de redes que pode ser adaptada de maneira bastante geral para detecção de simetrias.

Em nosso modelo, mapas globalmente e localmente acoplados são utilizados para a detecção de simetria de reflexão com relação a um eixo de reflexão que é dado. Uma vez que sempre se pode efetuar uma mudança de coordenadas (de modo que o eixo de reflexão coincida com o eixo $y$ das ordenadas), pode-se limitar a detecção de simetrias de reflexão com relação a esse eixo. Cada pixel de uma imagem plana é associado a um mapa 
logístico e os acoplamentos são usados para inserir a estrutura dos elementos da figura, o que leva os mapas correspondentes ao sincronismo ou não. O sistema indica se a figura é simétrica através do sincronismo das órbitas de todos os seus elementos.

\subsection{Descrição do modelo}

Como mostrado no capítulo 2, mapas parametrizados e globalmente acoplados (GCM) podem ser descritos pelo seguinte sistema de equações:

$$
x_{i}(t+1)=(1-\varepsilon) f_{\mu}\left(x_{i}(t)\right)+\frac{\varepsilon}{N} \sum_{i=1}^{N} f_{\mu}\left(x_{i}(t)\right)
$$

sendo $x_{i}(t)$ variáveis de estado calculadas no tempo discreto $t$. O termo $\sum_{i=1}^{N} f_{\mu}\left(x_{i}(n)\right)$ fornece o acoplamento global e conecta cada elemento a todos os demais. Aqui adotase $f_{\mu}(x)=\mu x(1-x)$, a função do mapa logístico com parâmetro $\mu$, e $\varepsilon$ é o termo de acoplamento do sistema e $N$ é o número total de mapas.

É comum, em modelos de redes baseados em mapas acoplados, empregar a abordagem de se associar um mapa a cada elemento que se deseja representar [Ito, 2000]. Essa abordagem também é adotada aqui. Cada mapa $i$, e sua variável de estado $x_{i}(t)$, correspondem a um pixel da imagem na qual se deseja identificar a presença de simetria de reflexão com respeito ao eixo das ordenadas $(y)$.

A partir modelo geral (3.4), fizemos duas modificações para permitir a detecção de simetria. Assim:

$$
x_{i}(t+1)=(1-\varepsilon) f_{\mu}\left(x_{i}(t)\right)+\frac{\varepsilon}{N} \lambda\left(x_{i}(t)\right) \sum_{i=1}^{N} f_{\mu}\left(x_{i}(t)\right)+I_{i}
$$

Introduzimos entradas externas $I_{i}$ para representar o valor de cada pixel da imagem, e adicionamos um termo de acoplamento local, $\lambda\left(x_{i}(t)\right)$, que busca levar ao sincronismo mapas associados a pixeis simétricos (a expressão de $\lambda\left(x_{i}(t)\right)$ é definida adiante).

Em modelos em que coexistem acoplamento global e local, em geral, as conexões globais tendem a tornar o sincronismo mais fácil de ser alcançado, enquanto as conexões locais buscam representar propriedades espaciais. Aqui, o uso da denominação local não 


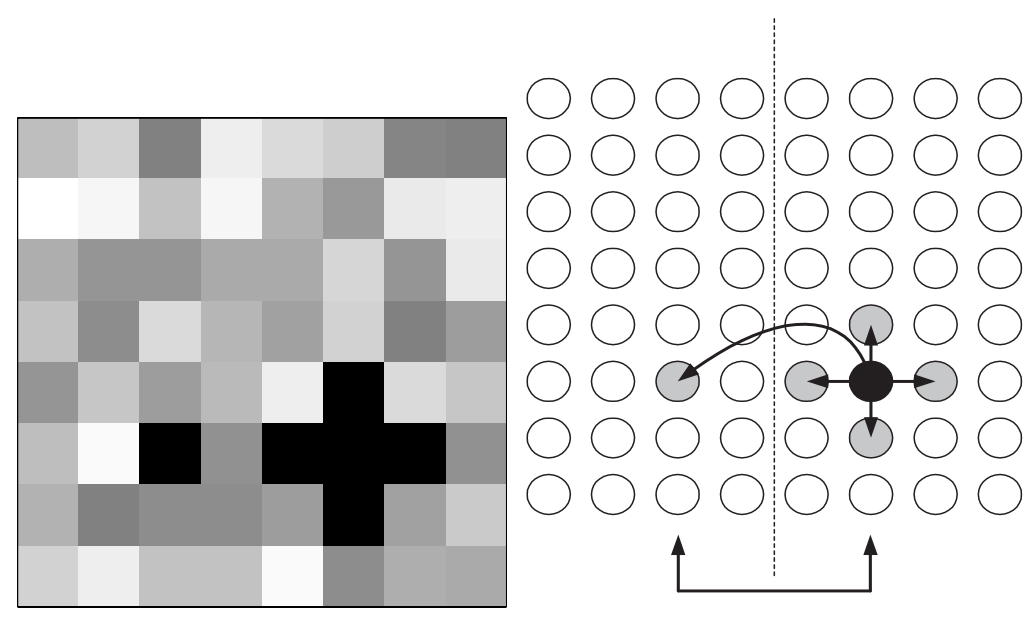

Figura 3.2: Representação dos pixeis de uma imagem por elementos da rede de mapas e o acoplamento "local" que busca informações sobre a simetria da imagem. Assim, cada mapa está conectado a quatro vizinhos próximos e ao mapa diametralmento oposto.

se refere (unicamente) à interação de elementos vizinhos, mas ao fato de estarem conectados elementos específicos, com uma relação espacial (de "localização") entre eles. Assim, colaboram, nesse termo de acoplamento local, elementos próximos e elementos diametralmente opostos ou "bastante" distantes, através dos quais se busca inserir a estrutura de simetria (ver figura 3.2).

Como interessa-nos o estudo de simetrias de reflexão no plano e sendo imagens $2 D$ representadas por matrizes de pixeis, as quais, por simplicidade, supomos matrizes quadradas $m \times m$, podemos reescrever a equação (3.5) de forma mais conveniente como:

$$
x_{i j}(t+1)=(1-\varepsilon) f_{\mu}\left(x_{i j}(t)\right)+\frac{\varepsilon}{N} \lambda\left(x_{i j}(t)\right) \sum_{i=1}^{m} \sum_{j=1}^{m} f_{\mu}\left(x_{i j}(t)\right)+I_{i j}
$$

sendo $m \times m$ o número de elementos da matriz quadrada que contém a imagem $2 D$. Os valores $i j$ indexam assim uma variável de estado $x_{i j}$, associada ao pixel linha $i$ e coluna $j$ de uma matriz. Note que índice $i$, em conjunto $i j$ em (3.6), não corresponde mais ao mesmo índice empregado anteriormente em (3.5), onde considerava-se apenas uma cadeia linear de pixeis.

\section{Correlação e laplaciano}

Agora, cada pixel na posição $i j$ corresponde a um único mapa $i j$ com a variável de estado $x_{i j}(t)$. O termo de acoplamento local $\lambda\left(x_{i j}(t)\right)$ é definido da seguinte maneira: 


$$
\lambda\left(x_{i j}(t)\right)=\lambda\left(x_{i, j}(t)\right)=x_{i, j}(t) x_{i, m-j+1}(t)+\Delta x_{i, j}(t)
$$

sendo $\Delta x_{i, j}(t)$ o operador laplaciano:

$$
\Delta x_{i j}(t)=x_{i+1, j}(t)+x_{i-1, j}(t)+x_{i+1, m-j+1}(t)+x_{i-1, m-j+1}(t)-4 x_{i j}(t)
$$

com $x_{i j}=0$ para quaisquer valores de $i, j$ tal que $i, j>m$ ou $i, j<0$. Assume-se que $m$ é par.

O acoplamento local utiliza duas operações. O produto na equação (3.7) representa a correlação [Ito, 2000] entre os mapas que representam pixeis simétricos, $x_{i, j}(t)$ e $x_{i, m-j+1}(t)$, com relação ao eixo $y$, e assume seu valor máximo quando os mapas apresentam suas dinâmicas sincronizadas, que aqui corresponde a $\left|x_{i, j}(t)-x_{i, m-j+1}(t)\right|=0$. O operador laplaciano (3.8) representa uma média ponderada dos estados na vizinhança de cada mapa (vizinhança de von Neumann, ver seção (2.7)). O laplaciano da função $u$ é uma expressão a derivadas parciais que, em coordenadas cartesianas e no plano, se escreve como $\nabla^{2} u=\partial^{2} u / \partial^{2} x^{2}+\partial^{2} u / \partial^{2} y^{2}$. Tal expressão surge freqüentemente em problemas de propagação [Bar-Yam, 1997] [Haberman, 1997]. Uma aproximação linear e discreta do laplaciano de $u$ na vizinhança de um ponto $\left(x_{0}, y_{0}\right)$ é [Haberman, 1997]:

$$
\nabla^{2} u\left(x_{0}, y_{0}\right)=\frac{u\left(x_{0}+\Delta x, y_{0}\right)+u\left(x_{0}-\Delta x, y_{0}\right)+u\left(x_{0}, y_{0}+\Delta y\right)+u\left(x_{0}, y_{0}-\Delta y\right)-4 u\left(x_{0}, y_{0}\right)}{(\Delta x)^{2}}
$$

Aqui emprega-se essa aproximação do operador laplaciano, correspondendo a um acoplamento na forma de vizinhança de von Neumann e com os pesos da ponderação, da influência de cada elemento, somando zero.

O modelo (3.6) leva ao sincronismo a dinâmica dos mapas associados a pixeis em posição simétrica com relação ao eixo $y$ e que tenham o mesmo valor $I_{i j}$. Assim, tende-se a $\left|x_{i, j}(t)-x_{i, m-j+1}(t)\right|=0$. Os demais não se tornam sincronizados. Da definição dada na seção (2.6), investigamos a sincronização do sistema observando a evolução temporal das variáveis:

$$
y_{i}(t)=\sum_{j=1}^{m}\left|x_{i j}(t)-x_{i, m-j+1}(t)\right|
$$




$$
z(t)=\sum_{i=1}^{m} y_{i}(t)
$$

A expressão (3.10), correspondente a um campo médio dos estados dos mapas da $i$-ésima linha horizontal da imagem (pixeis em linhas perpendiculares ao eixo vertical), indica se há ou não simetria de reflexão da linha com relação ao eixo $y$. A expressão (3.11), correspondente ao campo médio global dos estados do sistema, indica a existência ou não da simetria de reflexão na imagem.

A imagem apresenta simetria de reflexão com relação ao eixo das ordenadas se todas as linhas horizontais são simétricas com relação a esse eixo. No modelo, linhas simétricas levam $y_{i}(t) \rightarrow 0$ a medida que o tempo evolui, de modo que também $z(t) \rightarrow 0$. A figura (3.3) ilustra como o modelo informa a presença ou não de simetria nas imagens representadas.

\subsection{Simulações e resultados}

Foram realizadas quatro classes de simulações. Na primeira classe, utilizamos imagens aleatórias, com pixeis assumindo valores na escala de tons de cinza (de 0 a 255), sendo classificadas como simétricas, ou não, com relação à reflexão em torno do eixo vertical central da matriz de pixeis. Em uma segunda classe de simulações, verificamos a robustez do modelo na detecção de simetria, empregando imagens com ruído adicionado. Na terceira classe, verificamos a capacidade do modelo de lidar com imagens que se modificam no tempo (cenas dinâmicas). Por último, realizamos experimentos computacionais com imagens fotográficas e investigamos um caso "prático".

\section{Parâmetros e intervalos de valores}

Em todas as simulações que seguem, a menos de menção explícita de outros valores, empregamos: $\mu=3.5$ o parâmetro do mapa logístico, $\varepsilon=0.6$ o termo de acoplamento, e estados iniciais de cada mapa no intervalo [0 0.01$]$ atribuídos aleatoriamente. Os valores de cada pixel, assumindo valores de 0 a 255 na escala de cinzas, são normalizados no intervalo [0 0.01$]$ para definição das entradas $I_{i j}$. A escolha dos valores dos parâmetros, 
(a)
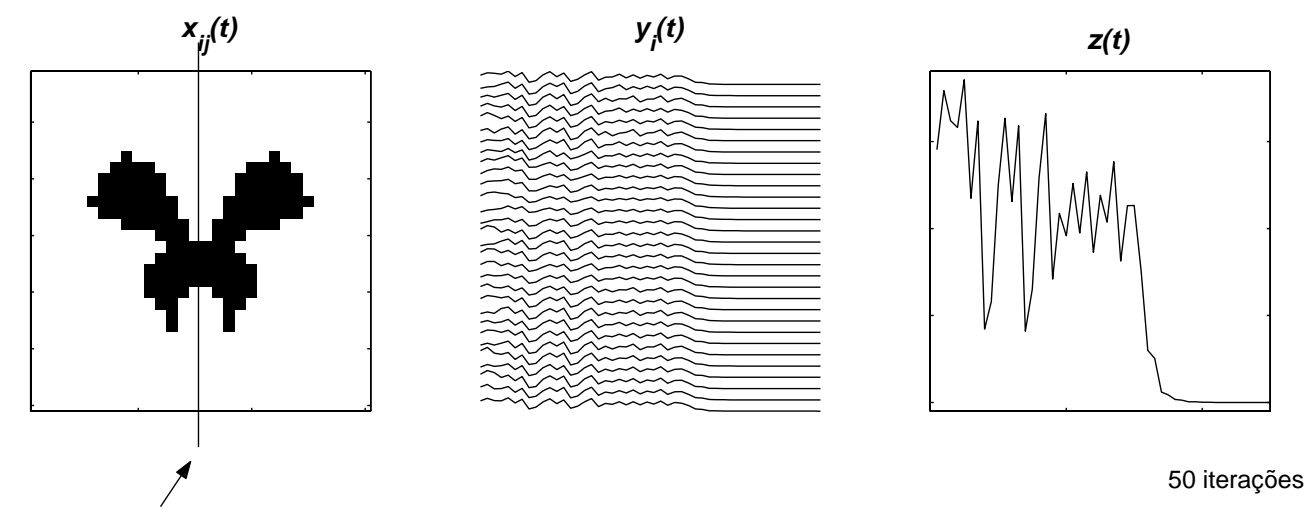

eixo para simetria vertical

(b)
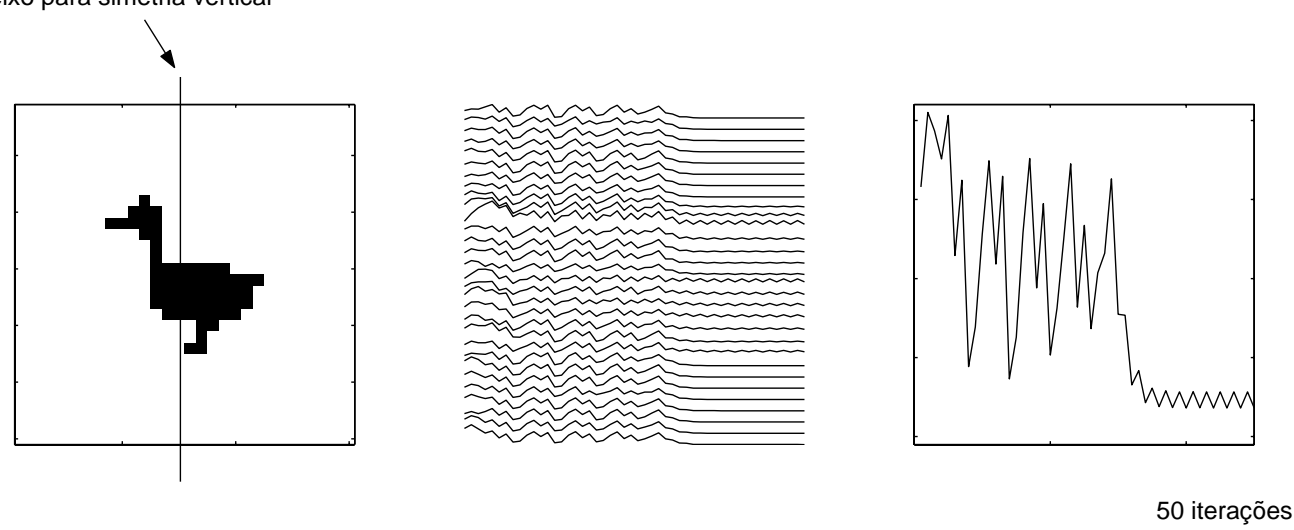

Figura 3.3: Figuras binárias (branco e preto) em matrizes $30 \times 30$ de pixeis. (a) A imagem da borboleta exibe simetria de reflexão com relação ao eixo das ordenadas, pois as órbitas $x_{i j}$ e $x_{i, m-j+1}$ sincronizam e os valores $y_{i}(t)$ e $z(t)$ tendem a zero. (b) A imagem do pato apresenta linhas não simétricas com relação à reflexão sobre o eixo das ordenadas, e os valores de $y_{i}(t)$ e $z(t)$ oscilam. 


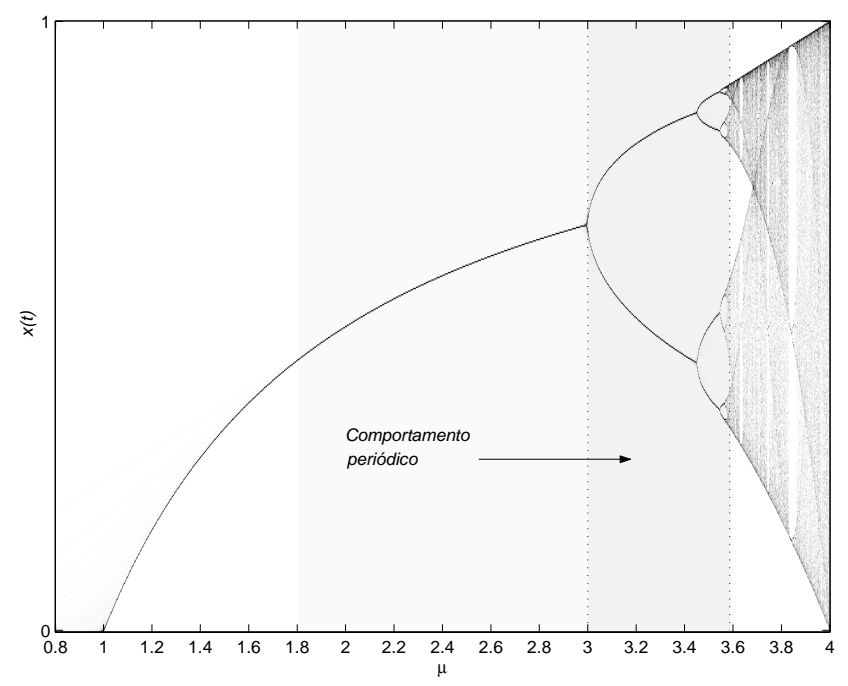

Figura 3.4: Mapa de bifurcação do mapa logístico. O valor do parâmetro de bifurcação do modelo é buscado a partir de valores do mapa isolado que apresentem o comportamento desejado para a rede. Nesse caso, buscam-se valores que levem o sistema a uma dinâmica periódica.

$\mu \mathrm{e} \varepsilon$, foi feita partindo-se dos valores do parâmetro do mapa isolado que apresentavam o comportamento desejado, tendo-se optado por uma dinâmica do tipo periódica. Assim, evitou-se valores que levassem a estados estacionários ou dinâmica caótica. Além de diagramas de bifurcação (ver figura (3.4)), foram empregados diagramas de pontos limite (ou seja, o conjunto $\omega$-limite do sistema ${ }^{2}$ ) e diagramas estado-tempo como os da figura (3.5) na investigação dos sincronismos do sistema.

Destacamos a importância da escolha dos parâmetros e dos intervalos de valores empregados. A escolha de outros valores pode conduzir o sistema para um estado coerente, ou completamente desordenado, para quaisquer entradas, simétricas ou não, caso em que o modelo falha na detecção de simetria. As simulações mostram que esses valores dependem principalmente da quantidade de entradas (tamanho da imagem), o que deve ser objeto de investigação futura. Por hora, são empregados apenas os valores experimentalmente obtidos.

A maior parte das simulações foi realizada com imagens aleatórias. Inicialmente, valores aleatórios são atribuídos a cada par de elementos simétricos com relação ao eixo vertical da matriz quadrada de pixeis. A imagem obtida é então simétrica. Foram produzidas imagens branco e preto (figura (3.3)) e imagens em tons de cinza (figuras (3.6) e (3.7)) - escala de cinza de $0=$ preto e $255=$ branco - com valores normalizados no intervalo

\footnotetext{
${ }^{2}$ Refere-se ao conjunto de pontos do regime permanente do sistema, em contraposição ao conjunto de pontos do regime transitório, $\alpha$-limite [Monteiro, 2002].
} 

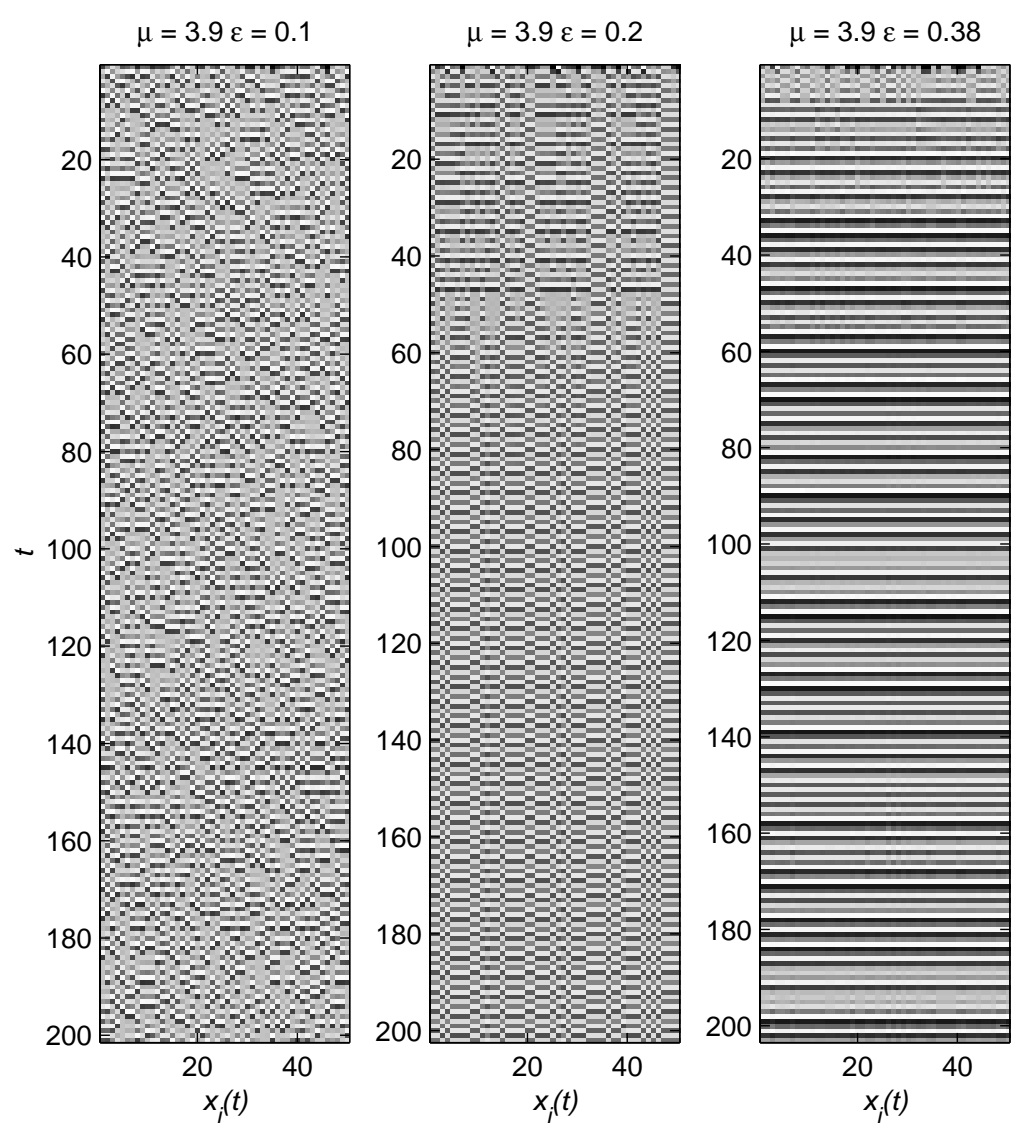

Figura 3.5: Diagramas estado-tempo, de uma rede de 50 mapas logísticos acoplados (GCM), apresentam a evolução dos estados de cada mapa. Uma escala de cinza é utilizada com os menores valores sendo representados por preto e os maiores valores por branco. Verifica-se, da esquerda para direita, que valores maiores do termo de acoplamento levam a um maior número de elementos sincronizados (colunas verticais semelhantes). Essa técnica de representação também pode ser encontrada em [Boccaletti et al., 2002] na análise de sistema estendidos. 
[0 0.01$]$ para determinação das entradas $I_{i j}$. Sobre as imagens inicialmente simétricas, foram adicionados diferentes níveis de assimetria alterando-se, aleatoriamente, os valores dos pixeis de cada imagem. Foram utilizadas matrizes com dimensões $m=6,8,10,20,30$. Para cada uma, foram efetuadas cerca de 50 realizações para imagens em branco e preto e imagens em tons de cinza. Em todas as simulações, conseguimos verificar com sucesso a presença ou não da simetria de reflexão com relação ao eixo vertical da matriz, tanto para cada linha de pixeis das figuras como para a figura completa. Parte dos resultados são mostrados na figura (3.6)(a) em que matrizes $8 \times 8$ de pixeis em tons de cinza exibem diferentes níveis de simetria corretamente identificados pelo modelo.

\section{Ruido}

O uso prático de uma técnica para a detecção de simetria requer a habilidade dessa técnica de identificar propriedades de simetria, mesmo na presença de ruído. Essa propriedade é também observada no modelo. Partindo-se de imagens simétricas, às quais adicionamos ruído, pudemos indentificar corretamente a presença de simetria nas imagens onde o ruído acrescido não alterou o aspecto simétrico da imagem. Mais precisamente, adicionamos valores aleotórios $\eta(i j)$ a diferentes porcentagens de entradas $I_{i j}$. Os valores adicionados foram produzidos com média $E(\eta(i j))=0.005$, correspondente ao valor médio do valor original das entradas, e variância $\sigma^{2}=10^{-5}$ (ver figura (3.6)).

\section{Cenas dinâmicas}

O modelo também apresenta uma propriedade importante: a de lidar com imagens que se modificam no tempo (cenas dinâmicas). Outros modelos de redes neurais, baseados em funções limiar, como modelos de Hopfield com elementos totalmente conectados [Hopfield, 1982], [Haykin, 1999], requerem a reinicialização da rede para novas entradas. Nosso modelo permite trabalhar com novas entradas, ou alterações dinâmicas na entrada inicial, sem a necessidade de reinicialização dos estados da rede. Dessa forma, a equação (3.6) passa a admitir entradas temporais tomando-se $I_{i j}$ como uma função do tempo:

$$
x_{i j}(t+1)=(1-\varepsilon) f_{\mu}\left(x_{i j}(t)\right)+\frac{\varepsilon}{N} \lambda\left(x_{i j}(t)\right) \sum_{i=1}^{m} \sum_{j=1}^{m} f_{\mu}\left(x_{i j}(t)\right)+\underline{I_{i j}(t)}
$$

A figura (3.7) exemplifica esse resultado. Nessa figura, uma imagem inicialmente 


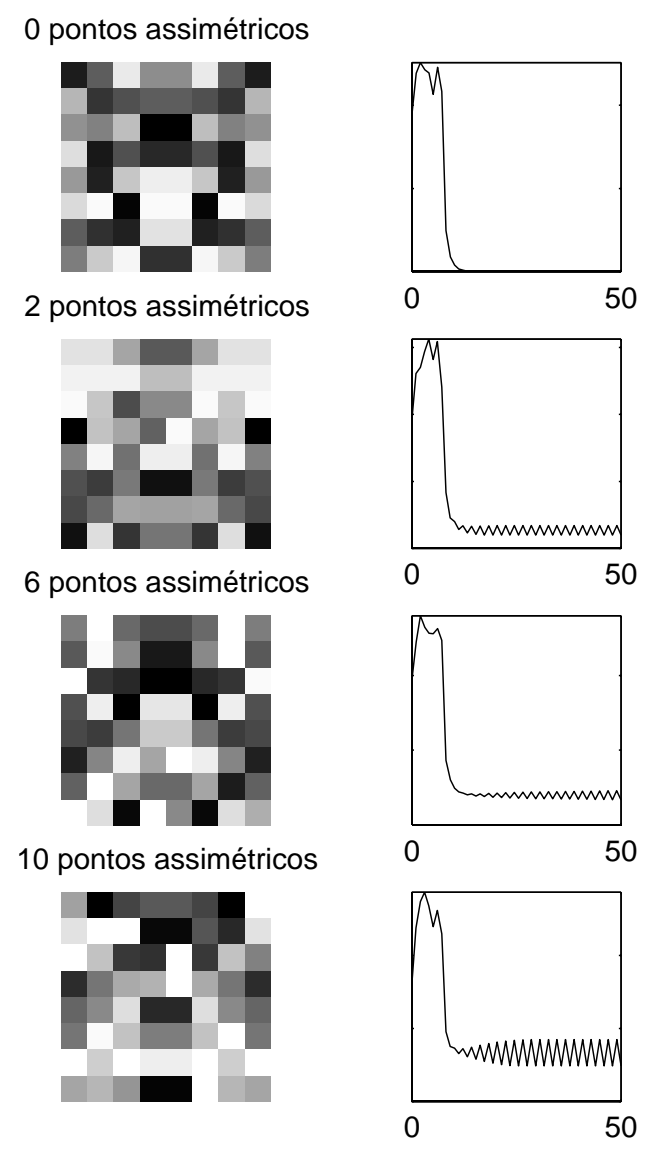

(a) Figuras com diferentes níveis de assimetria
$0 \%$

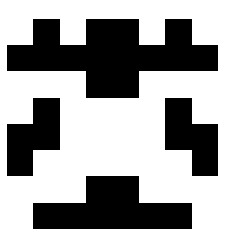

$2.5 \%$

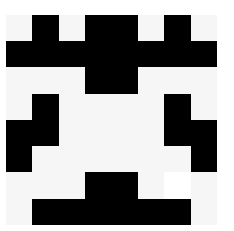

$10 \%$

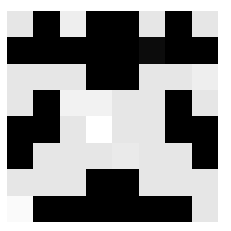

$20 \%$

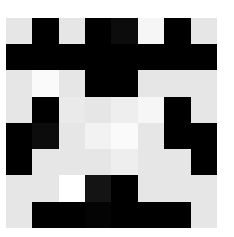

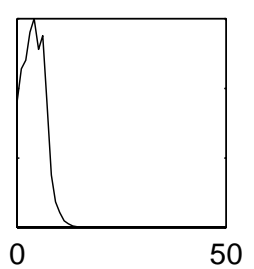
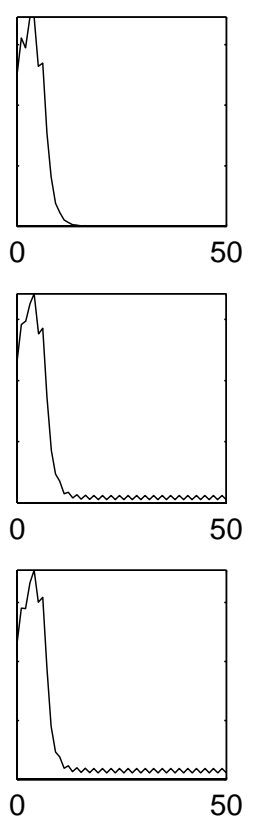

(b) Figuras com adição crescente de ruído

Figura 3.6: Matrizes de $8 \times 8$ pixeis. (a) Imagens em tons de cinza com diferentes níveis de assimetria vertical são corretamente classificadas. Quanto maior a assimetria, maior a amplitude da oscilação. (b) Diferentes imagens com adição de ruído $\eta(i j)$ a um número crescente de entradas $(0,2.5,10$ e $20 \%)$ são classificadas corretamente pelo modelo. O ruído adicionado tem média $E(\eta(i j))=0.005$ e variância $\sigma^{2}=10^{-5}$. 

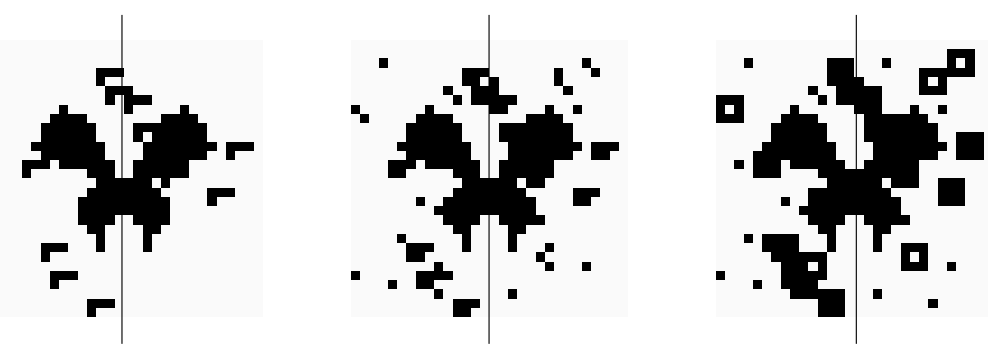

$\mathrm{t}<40$

$\mathrm{t}=40$

$t=60$
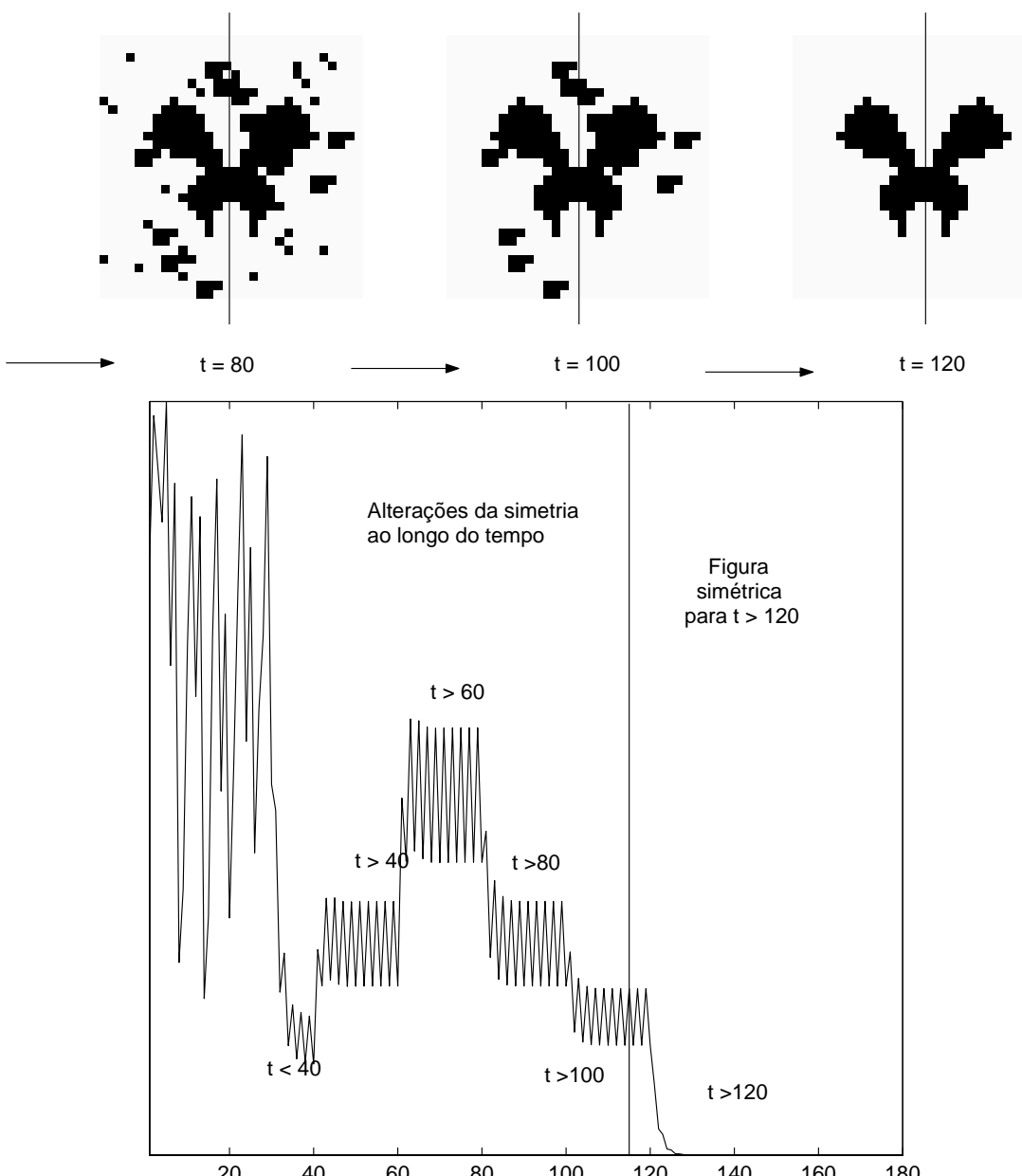

$t=120$

Figura 3.7: A imagem inicial é modificada para um maior nível de assimetria nos instantes $t=40$ e $t=60$, que são tempos discretos correspondentes aos passos da simulação de (3.12). Nos instantes $t=80$ e $t=100$, a imagem é modificada para níveis de simetria maior até tornar-se totalmente simétrica no instante $t=120$. Esse comportamento se reflete nos valores de $z(t)$ que indicam as alterações na imagem a cada instante de tempo. 


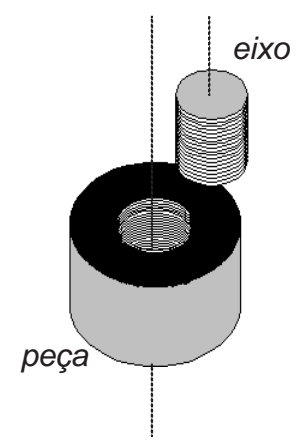

Figura 3.8: Aplicação para centralização de eixo.

assimétrica é modificada para um maior nível de assimetria nos instantes $t=40$ e $t=60$, tempos discretos do sistema (3.12) em que cada $t$ corresponde a um passo da simulação, através de alteração das entradas $I_{i j}(t)$. A seguir, nos instantes $t=80$ e $t=100$, a imagem é modificada para níveis de assimetria menor, até tornar-se simétrica no instante $t=120$.

Por último, aplicamos o modelo a fotos digitalizadas envolvendo um número maior de pixeis (figura (3.10)). Também, idealizamos uma aplicação de detecção de simetria simultaneamente sobre dois eixos para a solução de um problema de centralização de peças (figuras (3.8) e (3.9)).

\subsection{Discussão e modelos biológicos}

A hipótese de que nos processos de percepção e cognição o fenômeno do sincronismo desempenha um papel importante é, em geral, bastante aceita ${ }^{3}$. Oscilações neuronais síncronas têm sido observadas durante diferentes atividades do cérebro envolvendo audição [Campbell \& Wang, 1996], visão e olfato [Freeman, 1992]. A codificação espaço-temporal, com a sincronização de osciladores, pode oferecer vantagem computacional sobre os tradicionais modelos neurais de função limiar, especialmente para modelos biológicos, permitindo o tratamento de um maior número de informações em um menor tempo. Uma implementação comum de modelos temporais de correlação baseados em sincronismo é o uso de osciladores como elementos de uma rede (neural), em que cada um representa algum aspecto de um objeto [Campbell \& Wang, 1996], por exemplo, o pixel de uma imagem.

\footnotetext{
${ }^{3}$ Embora, em parte, isso tenha motivado o uso da sincronização em nosso modelo, deixamos claro que outros conceitos de sincronismo podem ser empregados em modelos biológicos.
} 

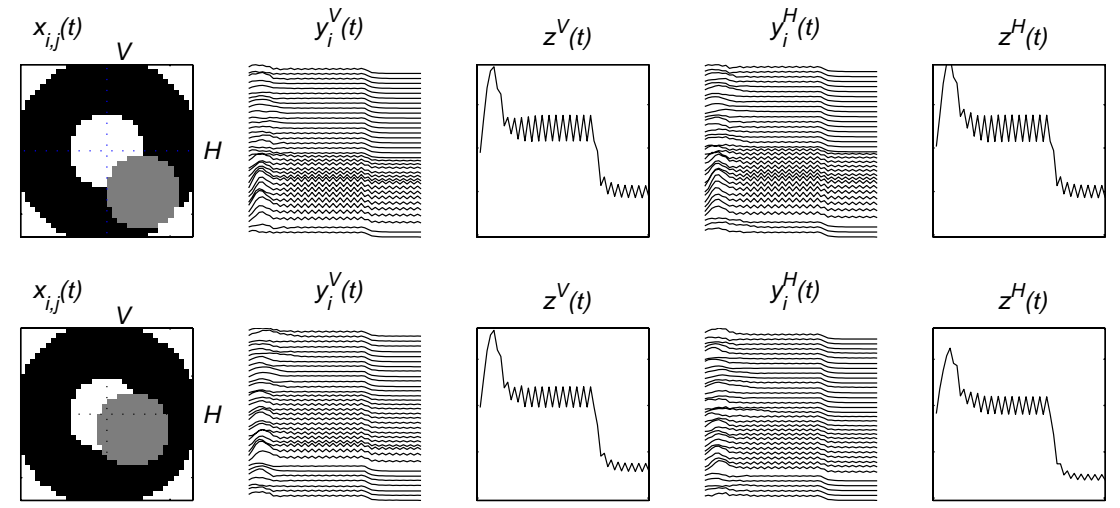

$y_{i}^{v}(t)$

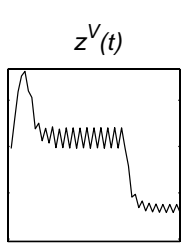

$y_{i}^{H}(t)$
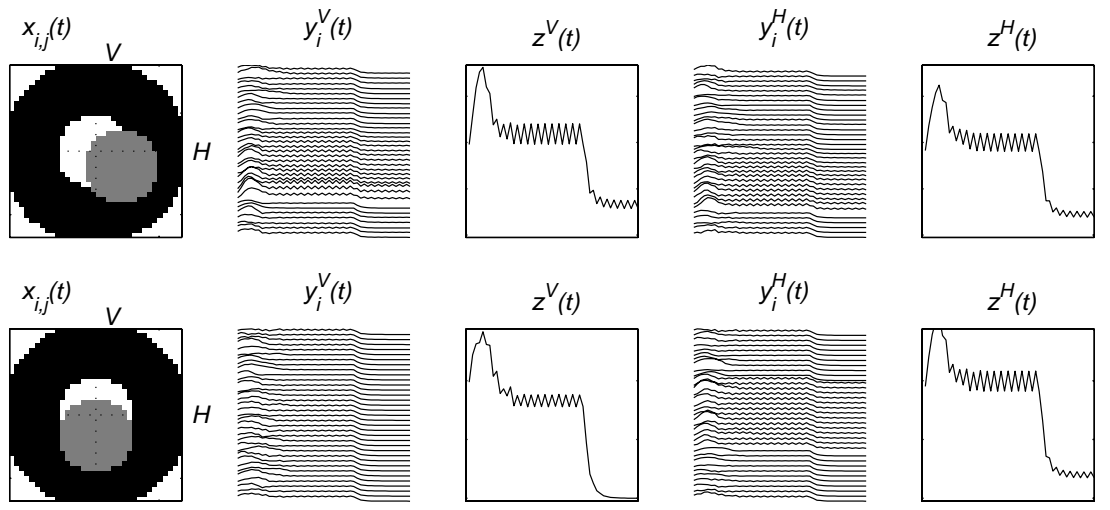

$y_{i}^{v}(t)$
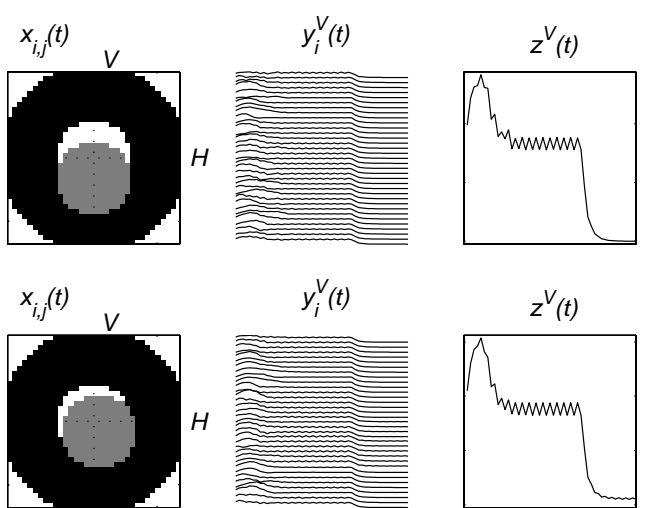

$y_{i}^{v}(t)$
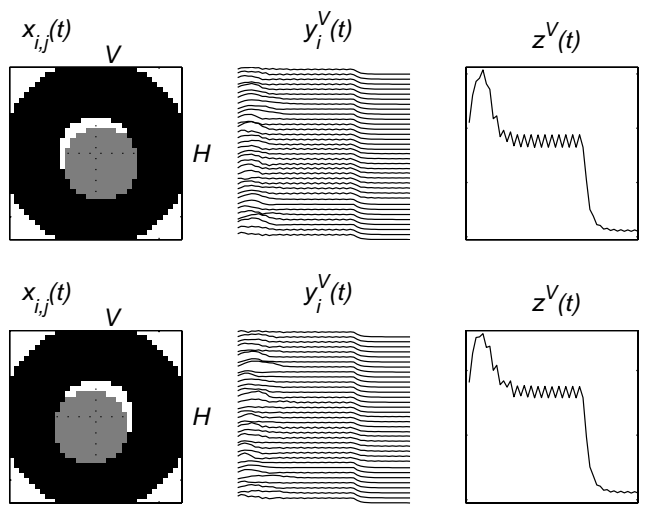

$y_{i}^{v}(t)$
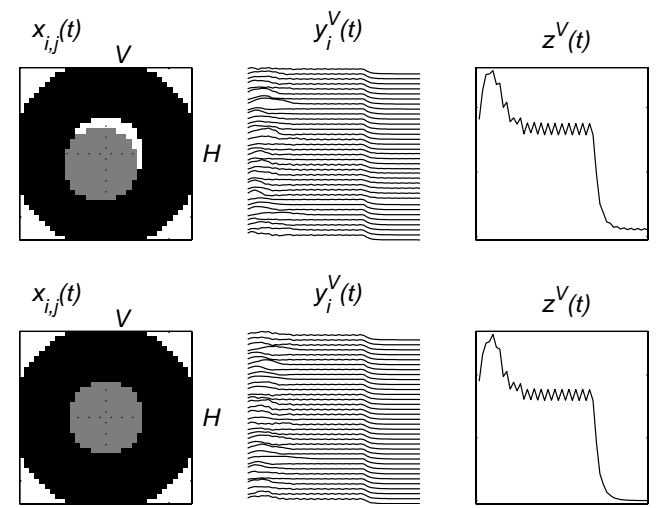

$y_{i}^{v}(t)$
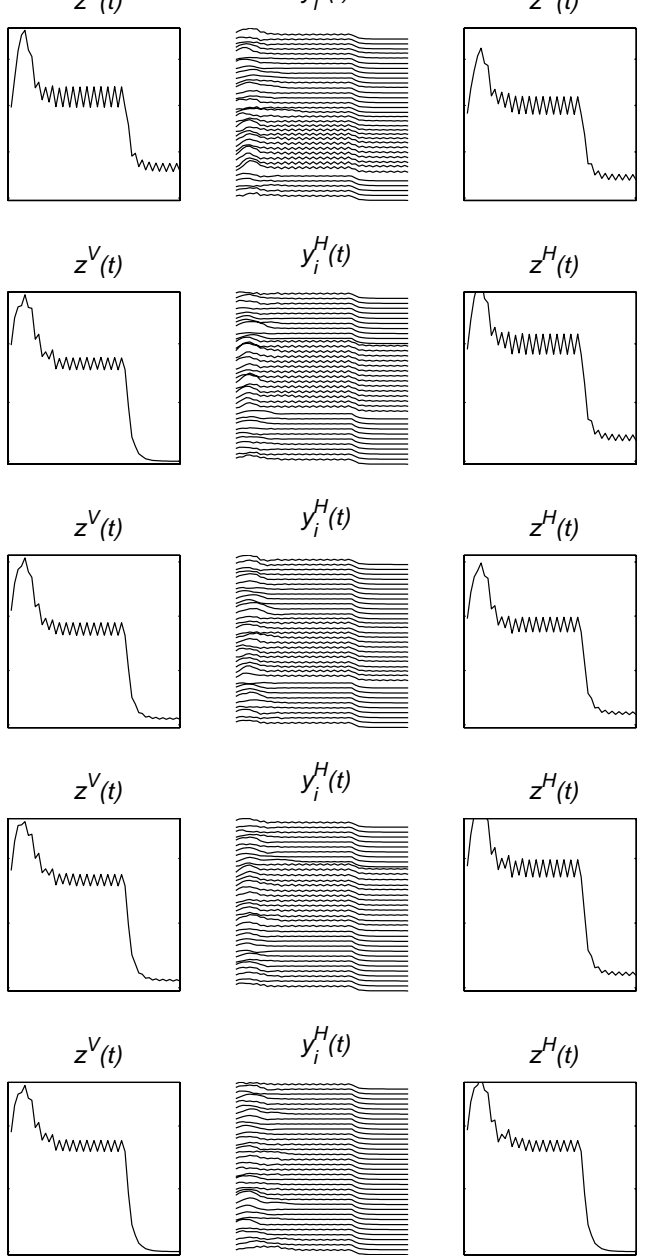

$y_{i}^{H}(t)$
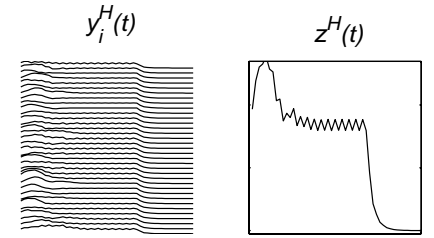

Figura 3.9: Centralização de eixo. O sistema é aplicado para detecção de simetria de reflexão com relação ao eixo vertical $V$ e ao eixo horizontal $H$ da matriz da imagem. Os sinais podem ser utilizados para se ajustar a posição da peça ao eixo (ver figura (3.8). As matrizes de pixeis são de $34 \times 34$. Os valores de entrada de cada pixel foram normalizados no intervalo $[00.1]$ e os parâmetros valem $\varepsilon=0.5$ e $\mu=3.2$. 


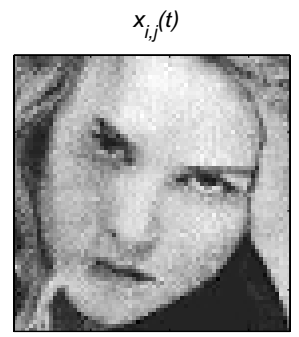

$x_{i, j}(t)$

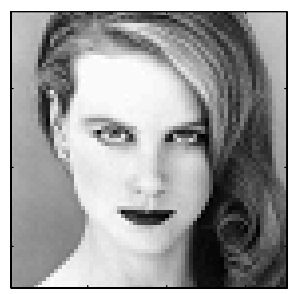

$x_{i, j}(t)$

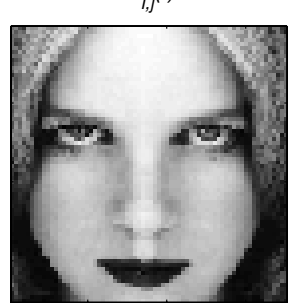

$x_{i, j}(t)$

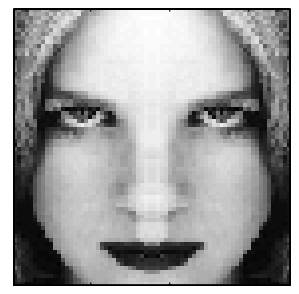

$y_{i}(t)$

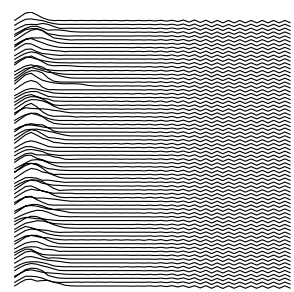

$y_{i}(t)$

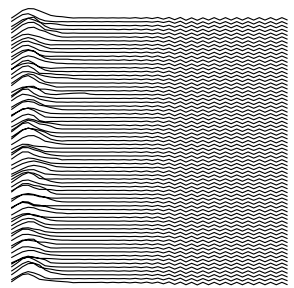

$y_{i}(t)$

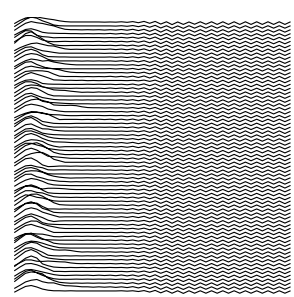

$y_{i}(t)$

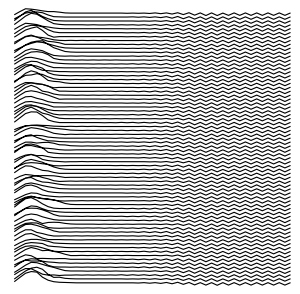

$z(t)$

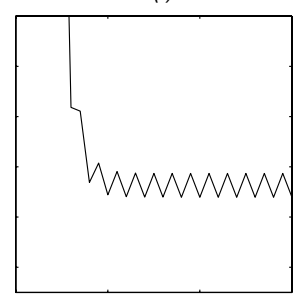

$z(t)$

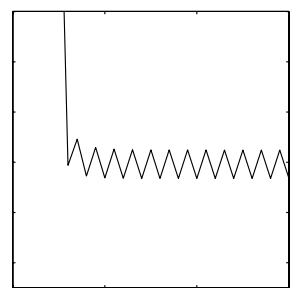

$z(t)$

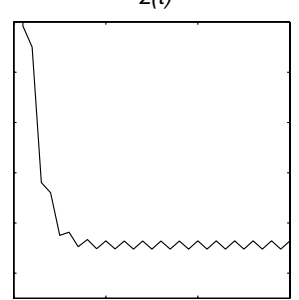

$z(t)$

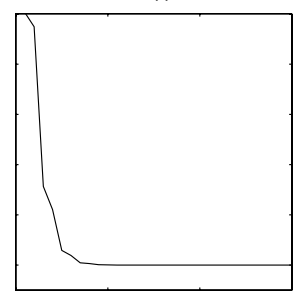

Figura 3.10: Verificação de simetria com relação ao eixo vertical sobre fotos da atriz Nicole Kidman para teste do modelo. As imagens encontram-se digitalizadas em matrizes de pixeis de $70 \times 70$. Os valores de entrada de cada pixel foram normalizados no intervalo [0 0.0001$]$ e os parâmetros valem $\varepsilon=0.5$ e $\mu=3.2$. As duas fotos mais abaixo foram manipuladas através de software para obtenção de imagens de rostos simétricos. Apenas no último a imagem é perfeitamente simétrica. 
Essas redes de osciladores, em geral, são implementadas sobre sistemas de tempo contínuo e alguns exemplos podem ser encontrados em [Hayashi, 1994], [Nishii, 1998], [Zhao et al., 2000] e [Chen \& Wang, 2002]. No nosso modelo, mapas acoplados são empregados para implementação de uma rede de osciladores em tempo discreto, um abordagem que também encontra-se em [Zhao, 2000b] [Zhao \& Macau, 2001] para problemas de segmentação de cenas, e que traz ao menos duas vantagens: ( $i$ ) torna-se mais adequado para a implementação computacional de que os modelos em tempo contínuo; $(i i)$ simplifica cada elemento da rede a um mapa unidimensional $(f: \mathbf{R} \rightarrow \mathbf{R})$ sendo, de fato, cada mapa equivalente (homeomórfico) a um modelo de neurônio excitatório-inibitório em tempo discreto [Wang, 1991] (ver exemplo (2.6)); (iii) permite entender cada elemento do modelo como um par de neurônios excitatório-inibitório ou populações desses neurônios.

\subsection{Conclusão e sumário}

Desenvolvemos, aqui, um modelo dedicado para a detecção de simetria de reflexão, sobre um eixo dado, através do sincronismo, ou não, das órbitas de seus elementos. O modelo envolve tanto acoplamentos locais como acoplamento global dos elementos. Buscamos, assim, inserir as relações espaciais nos acoplamentos locais e fazer uso da habilidade de acoplamentos globais levarem os elementos a estados sincronizados. Diferentes imagens foram corretamente classificadas, verificando-se também a capacidade de tratamento de imagens com ruído e cenas diâmicas. Ao final, destacamos as vantagens do uso de mapas em comparação aos modelos de tempo contínuo e modelos neurais tradicionais. Suas aplicações podem requerer, estando o modelo limitado a um único eixo de reflexão, translação das imagens ou o uso de um novo conjunto de equações para cada eixo adicional (ver exemplo da figura (3.9)). A principal limitação do presente modelo parece residir na obtenção de valores adequados dos parâmetros ${ }^{4}$.

\footnotetext{
${ }^{4}$ Para detalhes sobre técnicas de adequação de valores de parâmetros e de codificação de entradas em sistemas de mapas acoplados ver [Del-Moral, 2001], [Del-Moral, 2003].
} 


\section{Sistemas auto-organizáveis}

Art, literature, and music create order. Science searches for order that already exists.

Anônimo

Parte deste capítulo baseia-se no trabalho de [Oliveira \& Monteiro, 2003]. Aqui, investiga-se o surgimento de clusters, como definidos na seção (2.7), em redes de mapas acoplados com topologias de acoplamento não regulares ${ }^{1}$.

\section{Auto-organização}

Inicia-se delineando o conceito de auto-organização.

Auto-organização pode ser considerado um processo no qual um padrão em nível global de um sistema (nível macroscópico) surge unicamente por numerosas interações entre seus elementos (nível microscópico) e as regras de interação realizam-se fazendo uso somente de informações locais, sem referência ao padrão global [Camazine et al., 2001]. Essa definição de auto-organização, parece bastante adequada para nossos propósitos. Em sistemas de mapas acoplados do tipo CML (acoplamentos dos vizinhos mais próximos), cada elemento (nível microscópico) exibe uma dinâmica própria e interage unicamente com os vizinhos locais. Após várias iterações, o sistema (nível macroscópico) passa a exibir um estado $(i)$ coerente, $(i i)$ ordenado, com a formação de clusters, ou (iii) completamente ou totalmente dessincronizado (ver seção (2.7). A exemplo de [Kaneko, 1994], [Kaneko \& Tsuda, 2000], é nesse sentido que se emprega aqui o termo auto-organização. Particularmente, interessa o surgimento do estado (ii) ordenado, em que são formados $k$ grupos de elementos, com $k>1$ mas menor que o número de elementos da rede, exibindo órbitas sincronizadas.

\footnotetext{
${ }^{1}$ Consideram-se topologias de acoplamento regulares aquelas na qual todos os elementos exibem o mesmo formato de acoplamento, como um padrão de conexão entre vizinhos mais próximos, idêntico para todos os elementos; ou a conexão completa de todos os elementos - ver seção (2.7).
} 


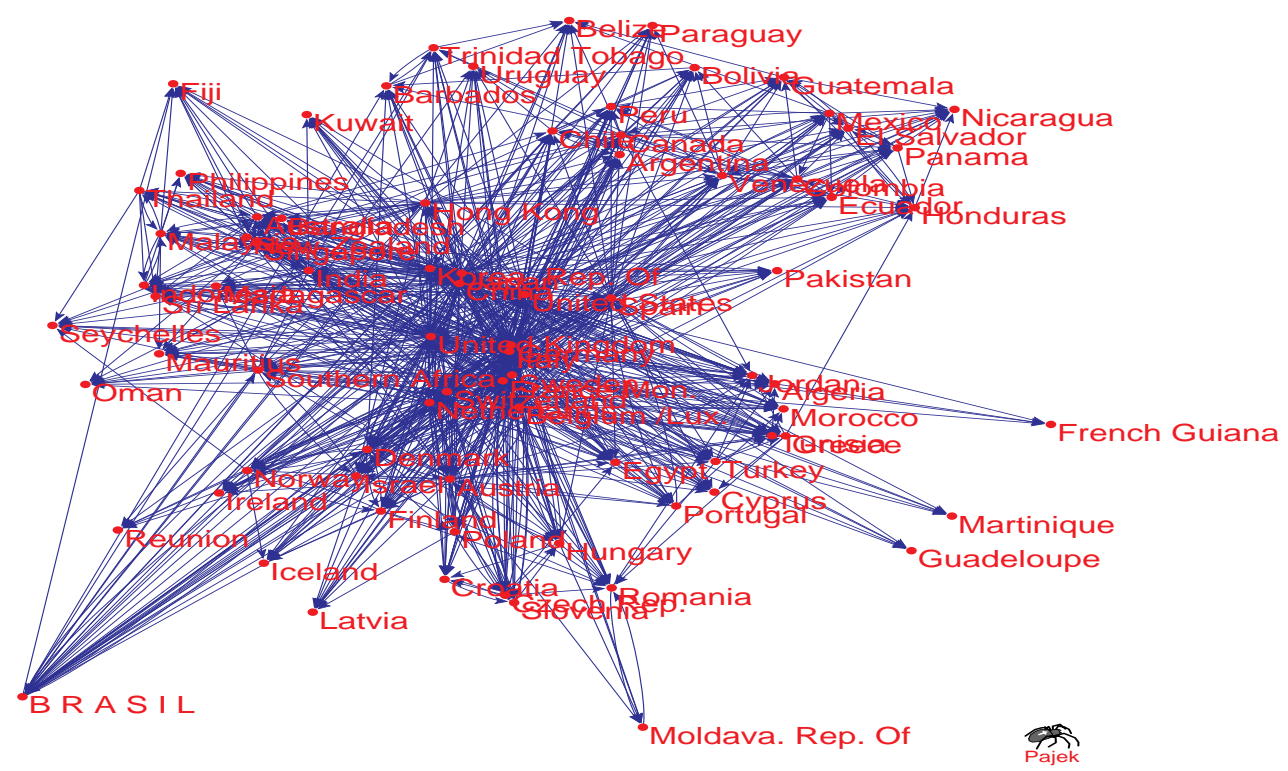

Figura 4.1: Uma rede com 80 vértices representa algumas relações no comércio mundial. A rede não é regular, pois os nós apresentam diferentes quantidades de conexões (construída com o software Pajek [Pajek, 2003]).

\subsection{Redes complexas}

Redes surgem, com freqüência, em uma série de sistemas e, em particular, em sistemas complexos. Surgem na biologia em redes de proteínas, conexões neurais e no metabolismo de células. Aparecem em fenômenos sociais, como redes de amigos, contatos sexuais, labor networks e redes de colaboração (atores em um filme ou referências de autores em um trabalho científico). Surgem no desenho de circuitos eletrônicos, em malhas de distribuição de energia e telefonia, em redes lingüísticas de sinônimos de palavras e em redes de relacionamento entre classes de uma linguagem de programação. E estão presentes na world wide web, em redes de hyperlinks entre documentos, de roteadores ou emails. Os exemplos são inúmeros e referências são encontradas em [Watts, 1999], [Strogatz, 2001], [Albert \& Barabási, 2002], [Barábasi, 2002], [Solé et al., 2003], [White \& Houseman, 2003]. Aqui, basta verificar que a importância dessas redes, e o seu aparecimento em diferentes contextos, justificam seu estudo.

Embora amplamente empregados na modelagem de uma série de fenômenos, como processos de difusão, dinâmica de populações, formação de padrões e redes biológicas, as redes com topologias regulares como as utilizadas em mapas localmente acoplados (CML) ou globalmente acoplados (GCM) parecem ser pouco plausíveis para representar a conectividade presente nas redes complexas, caracterizadas, dentre outros aspectos, por 
um grande número de nós conectados com diferentes números de conexões. Resultados empíricos sobre essas redes complexas mostram a necessidade de modelos mais adequados para sua representação.

Três modelos parecem melhor representar esses tipos de redes. Modelos de redes aleatórias, nas quais as conexões em um conjunto de nós são realizadas de forma aleatória, são conhecidos há mais de 40 anos [Albert \& Barabási, 2002] e eram, até muito recentemente, o modelo mais empregado. Nos últimos anos, modelos de redes sem escala [Albert \& Barabási, 2002], [Barábasi \& Bonabeau, 2003] e modelos de redes small-world [Watts \& Strogatz, 1998], [Strogatz, 2001] vêm sendo empregados como estruturas que melhor representam diversas características das redes complexas encontradas.

A seguir, busca-se caracterizar esses modelos, através de conceitos de grafos e medidas da topologia de redes, comparando-os com os modelos regulares de conexões até agora utilizados neste trabalho (CML e GCM).

\subsection{Grafos}

\section{Grafos, vértices e arestas}

A forma com que os elementos de uma rede encontram-se conectados constitui sua topologia. Para caracterização da topologia de uma rede, é conveniente introduzir a definição de grafo. Um grafo $G$ é um par $G=(V, A)$ de conjuntos $V$ e $A$, sendo $V$ um conjunto de vértices (ou nós) e $A$ um conjunto de arestas (ou conexões) entre os vértices (ver figura (4.2)). Denominam-se vizinhos aqueles vértices que se encontram conectados por uma aresta. O caminho entre dois vértices $v_{a}$ e $v_{b}$ em $G$ é um par $\left(V^{\prime}, A^{\prime}\right), V^{\prime} \subset V$ e $A^{\prime} \subset A$, tal que $v_{a}$ e $v_{b}$ encontram-se conectados. O grafo da figura (4.2) é assim definido por $G=(\{a, b, c, d\},\{(a, b),(a, d),(a, c),(b, d)\})$ : o vértice $a$ apresenta três vizinhos, $\{b, c, d\}$, e $G^{\prime}=(\{a, b, d\},\{(a, b),(b, d)\})$ é um caminho entre os vértices $a$ e $d$. Por fim, a distância entre dois vértices é o menor número de arestas que ligam esses vértices por algum caminho. Na figura (4.2), a distância dos nós $a$ e $d$, que estão diretamente conectados, é 1 , embora exista um outro caminho entre eles ${ }^{2}$.

\footnotetext{
${ }^{2}$ Consideram-se, aqui, unicamente grafos não direcionados, em que não se diferenciam os vértices $(a, b)$ e $(b, a)$, e sem peso para as conexões, isto é, dois caminhos entre dois vértices são equivalentes se possuem o mesmo número de arestas. O conceito de caminho também é aqui simplificado e definições mais elaboradas sobre grafos podem ser encontradas em [Lucchesi et al., 1979].
} 


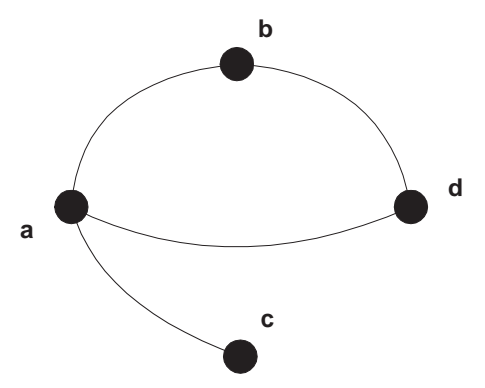

Figura 4.2: Grafo $G=(V, A)$ não-direcionado com vértices $V=\{a, b, c, d\}$ e arestas $A=\{(a, b),(a, d),(a, c),(b, d)\}$.

Como uma rede pode ter sua topologia descrita por um grafo, os termos rede e grafo são aqui empregados como sinônimos.

\subsection{Medidas para redes complexas}

Três conceitos, e as medidas a eles associadas, permitem melhor caracterizar as redes complexas. São eles: a distribuição de conexões, o menor caminho médio e o coeficiente de agregação ${ }^{3}$ de uma rede.

\section{Distribuição de conexões}

Considerando-se redes em que os elementos diferenciam-se unicamente pela forma, ou topologia, de suas conexões, a característica mais simples de um nó é o seu grau, definido como o número conexões $k_{i}$ que o nó $i$ apresenta. O grau médio de uma rede, $\bar{k}$, é a média dos valores $k_{i}$ para todos os nós. Assuma que os valores $k_{i}$ obedecem a uma distribuição de conexões, $P(k)$, que é a probabilidade de um nó selecionado, aleatoriamente na rede, apresentar exatamente $k$ conexões. Como consideram-se aqui redes em que dois elementos estão ou não conectados, não existindo múltiplas conexões entre dois nós, o grau de um nó corresponde também ao seu número de vizinhos.

\section{Menor caminho médio}

A distância $L_{i j}$ entre dois nós, $i$ e $j$, é definida como o número de arestas ao longo do menor caminho que conecta esses dois nós. O menor caminho médio, L, é então a média da distância entre dois nós, para todos os pares de nós da rede:

\footnotetext{
${ }^{3}$ Em inglês, os termos aparecem como: degree distribution, average path lenth, clustering coefficient.
} 


$$
L=\frac{1}{N} \sum_{i, j=j, i \in G} L_{i j}
$$

sendo $N$ o número de elementos da rede (grafo) $G$ e $L_{i j}$ a distância entre os nós $i, j=j, i$, com cada par computado uma única vez.

\section{Coeficiente de agregação}

Suponha um nó $i$ com $k_{i}$ arestas e, portanto, conectado a $k_{i}$ outros nós. Podem, então, existir $k_{i}\left(k_{i}-1\right) / 2$ arestas entre esses nós, o que ocorre somente se todos os nós vizinhos de $i$ são também vizinhos entre si. O coeficiente de agregação $C_{i}$ do nó $i$ é a fração do número de arestas que efetivamente existem entre os $k_{i}$ nós vizinhos do nó $i, E_{i}$, e o total de arestas que poderiam existir entre eles, $k_{i}\left(k_{i}-1\right) / 2$. Assim: $C_{i}=E_{i} /\left(k_{i}\left(k_{i}-1\right) / 2\right)$. Define-se o coeficiente de agregação da rede como o valor $C$, dado pela média dos valores $C_{i}$ sobre todos os nós da rede.

\subsection{Propriedades dos modelos}

\section{Distribuição de conexões}

A figura (4.3) mostra as distribuições de conexões características de redes regulares, aleatórias e sem escala. Redes regulares apresentam um número fixo de conexões por nó. Em uma rede de mapas do tipo GCM com $N$ elementos, cada nó encontra-se conectado a todos os demais e tem-se $k_{i}=\bar{k}=N$ para todo $i$. Em uma rede de mapas do tipo CML, com $N$ elementos e cada nó conectado a $n$ vizinhos, tem-se $k_{i}=\bar{k}=n$ para todo i. O gráfico da distribuição $P(k)$ assume, portanto, a forma de uma reta perpendicular ao eixo das abcissas (figura (4.3)). Nas redes aleatórias, o número de conexões segue a distribuição de Poisson [Albert \& Barabási, 2002]:

$$
P(k)=\frac{\bar{k}^{k} e^{-\bar{k}}}{k !}
$$

As redes sem escala exibem uma distribuição de conexões em forma de lei de potências [Albert \& Barabási, 2002], [Wang \& Chen, 2003]: 
Distribuição do número de conexões em redes regulares e aleatórias

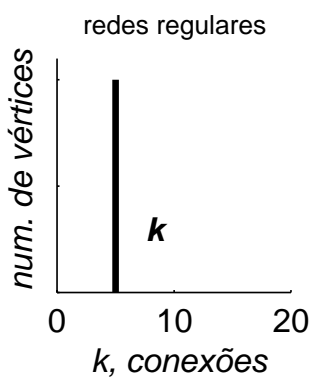

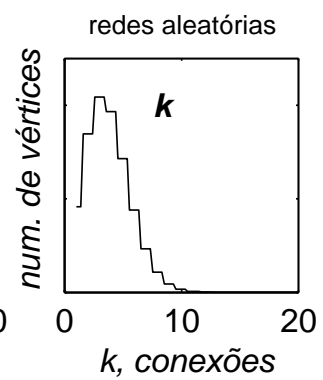

Distribuição do número de conexões em redes sem escala

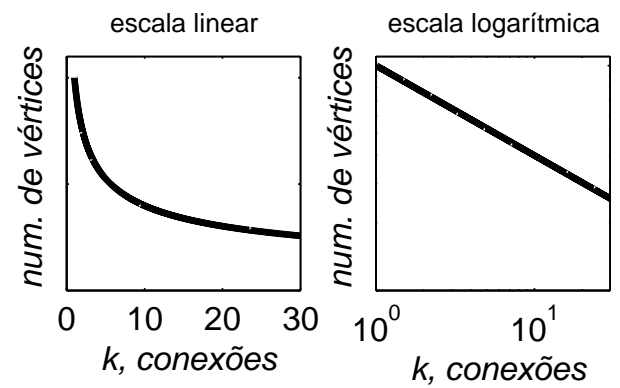

Figura 4.3: Distribuição do número de conexões (número de nós vizinhos) em redes regulares, aleatórias (redes small-world aqui incluídas) e sem escala. Modelos regulares apresentam uma distribuição uniforme de conexões. Em modelos aleatórios, o número de conexões segue uma distribuição de Poisson. Por último, em redes sem escala, o número de conexões obedece a uma distribuição de potências.

$$
P(k)=k^{-\gamma}
$$

sendo $\gamma$ o expoente de distribuição da rede.

A descoberta de que diversas redes complexas apresentam uma distribuição em forma de lei de potências é recente, tendo sido primeiramente observada no mapeamento de hyperlinks na world wide web por [Albert et al., 1999], quando se esperava encontrar a mesma distribuição das redes aleatórias. Posteriormente, observou-se essa distribuição em outras redes, como as redes de roteadores da internet, de proteínas, de conexão de empresas de biotecnologia etc. ([Barábasi, 2002], [Barábasi \& Bonabeau, 2003]). A forma da distribuição de lei de potências mostra que as redes sem escala possuem um pequeno número de nós com grande número de conexões.

Por último, modelos de rede small-world, como é visto mais adiante, são construídos, aleatoriamente, a partir de uma rede regular e exibem a mesma distribuição de conexões que as redes aleatórias.

\section{Menor caminho médio}

Se $G$ é uma rede totalmente conectada, como em um acoplamento de mapas do tipo GCM, então $L=1$ constante para qualquer número de elementos. Já em um acoplamento do 


\begin{tabular}{|c|c|c|c|c|c|}
\hline rede complexa & $\begin{array}{c}\text { número } \\
\text { de elementos } \\
N\end{array}$ & $\begin{array}{c}\text { coeficiente de } \\
\text { agregação } \\
C\end{array}$ & $\begin{array}{c}\text { menor caminho } \\
\text { médio } \\
L\end{array}$ & $\ln (N)$ & $k / N$ \\
\hline Internet (domínios) ${ }^{1}$ & 32711 & 0,24 & 3,56 & 4,51 & - \\
\hline Internet (roteadores) ${ }^{1}$ & 228298 & 0,03 & 9,51 & 5,36 & - \\
\hline Internet (hyperlinks) ${ }^{1}$ & 153127 & 0,11 & 3,1 & 5,19 & - \\
\hline Internet (emails) ${ }^{1}$ & 56969 & 0,03 & 4,95 & 4,76 & - \\
\hline Software (classes Java) ${ }^{1}$ & 1376 & 0,06 & 6,39 & 3,14 & - \\
\hline Circuitos eletrônicos ${ }^{1}$ & 329 & 0,34 & 3,17 & 2,52 & - \\
\hline Linguagem $^{1}$ & 460902 & 0,437 & 2,67 & 5,66 & - \\
\hline Autores (matemática) ${ }^{1}$ & 70925 & 0,59 & 9,5 & 4,85 & - \\
\hline Redes de alimentos ${ }^{1}$ & 154 & 0,15 & 3,4 & 2,19 & - \\
\hline Rede de energia $^{2}$ & 4941 & 0,08 & 18,7 & 3,69 & 0,00054 \\
\hline C.elegans ${ }^{2}$ & 282 & 0,28 & 2,65 & 2,45 & 0,04965 \\
\hline Atores de filmes ${ }^{2}$ & 225226 & 0,79 & 3,65 & 5,35 & 0,00027 \\
\hline
\end{tabular}

Tabela 4.1: Valores de coeficientes de agregação, $C$, e menor caminho médio, $L$, medidos em algumas redes complexas. Os valores comparativos de $\ln (N)$ e $k / N$ estão próximos aos obtidos em modelos de redes aleatórias. Os dados das três primeiras colunas encontramse compilados em (1) [Wang \& Chen, 2003] e (2) [Watts \& Strogatz, 1998] nos quais há maiores detalhes e referências sobre cada uma das medidas realizadas.

tipo CML, $L$ é função do número de elementos da rede $N$ e do número de vizinhos $k$ que se encontram conectados. Verifica-se que $L=\frac{N(N+k+2)}{2 k(N-1)}$ [Watts, 1999]. De modo mais geral, é aqui suficiente considerar que $L$ varia proporcionalmente (isto é, em escala) com respeito a $N$ (para $N$ suficientemente grande) e inversamente com respeito a $k$ e, portanto, $L \rightarrow \infty$ quando $N \rightarrow \infty$. Em redes complexas, entretanto, obtem-se valores muito pequenos de $L$, ou seja $L \ll N$, mesmo em redes com $N$ muito grande (ver tabela (4.1)). Os modelos de redes aleatórias, small-world e sem escala buscam representar isso fornecendo um menor caminho médio de ordem de $\ln N$, bastante próximo às medidas feitas em redes complexas (ver [Strogatz, 2001], [Albert \& Barabási, 2002], [Wang \& Chen, 2003]).

\section{Coeficiente de agregação}

Verifica-se que $C \leq 1$, sendo que $C=1$ somente se a rede é totalmente conectada (como em um acoplamento do tipo GCM). Já em redes do tipo k-vizinhos mais próximos, empregadas nos acoplamentos CML, o valor de $C$ pode ser obtido analiticamente por $C=\frac{3(k-2)}{4(k-1)}$, para $k \geq 2$ [Watts, 1999].

Redes complexas parecem ser caracterizadas por coeficientes de agregação elevados $(\sim 0.3)$. Os modelos aleatórios, entretanto, exibem um baixo coeficiente de agregação $C \sim \bar{k} / N$ [Watts \& Strogatz, 1998] (ver tabela (4.1)) não constituindo-se, portanto, em 
bons modelos. Os modelos de rede small-world são construídos de forma a representar uma rede com um coeficiente de agregação mais próximo das redes regulares. Sendo as redes small-world obtidas a partir de uma probabilidade dada $p$ (ver mais adiante), busca-se, nos modelos small-world, $C(p) \gg \bar{k} / N$ [Watts \& Strogatz, 1998].

Já nos modelos de rede sem escala, não há forma analítica para a obtenção do coeficiente de agregação, embora simulações numéricas [Albert \& Barabási, 2002] evidenciem que tais modelos forneçam um valor algumas vezes $(\sim 5)$ maior que as redes aleatórias.

Um sumário desses resultados encontra-se a seguir:

\begin{tabular}{c|cccc} 
& $\begin{array}{c}\text { redes } \\
\text { regulares }\end{array}$ & $\begin{array}{c}\text { redes } \\
\text { small-world }\end{array}$ & $\begin{array}{c}\text { redes } \\
\text { aleatórias }\end{array}$ & $\begin{array}{c}\text { redes } \\
\text { sem escala }\end{array}$ \\
\hline \multirow{2}{*}{$L(k)$} & $\begin{array}{c}P_{c m l}(k)=\bar{k} \\
P_{g c m}(k)=N\end{array}$ & $P(k)=\frac{\bar{k}^{k} e^{-\bar{k}}}{k !}(2)$ & $P(k)=\frac{\bar{k}^{k} e^{-\bar{k}}}{k !}(2)$ & $P(k)=k^{-\gamma}(2)$ \\
$C$ & $L_{c m l}=\frac{N(N+k-2)}{2 k(N-1)} \sim N(1)$ & $L \sim \frac{\ln (N)}{\ln (k)}$ & $L \sim \frac{\ln (N)}{\ln (k)}$ & $L \sim \frac{\ln (N)}{\ln (k)}$ \\
$L_{g c m}=1$ & & & \\
& $C_{c m l}=\frac{3(k-2)}{4(k-1)}(1)$ & $C(p) \gg \bar{k} / N(4)$ & $C \sim \bar{k} / N$ & $(3)$ \\
$C_{g c m}=1$ & &
\end{tabular}

- (1) Ver [Watts, 1999]. ${ }^{(2)}$ Ver [Albert \& Barabási, 2002]. ${ }^{(3)}$ Não há previsão analítica, embora dados empíricos forneçam evidências de que redes sem escala apresentem um coeficiente de agragação maior que as redes aleatórias. (4) O coeficiente de agregação em um modelo de rede small-world depende da probabilidade $p$ de religação (ver mais adiante) [Watts \& Strogatz, 1998].

\section{Redes complexas: small-worlds ou sem escala?}

As estruturas descritas permitem modelar redes cujos valores dos parâmetros característicos se aproximam dos encontrados empiricamente. Particularmente, os dados da tabela acima mostram que redes complexas "reais", no conjunto das propriedades de distribuição de conexões, menor caminho médio e coeficiente de agregação, parecem ser melhor representadas por modelos do tipo sem escala e small-world. Basicamente, entre 
outros fatores, as redes regulares não apresentam a mesma distribuição de conexões das redes complexas "reais". Acoplamentos do tipo GCM têm grau muito elevado e acoplamentos do tipo CML apresentam um menor caminho médio que cresce proporcionalmente com o tamanho da rede. As redes aleatórias, embora apresentem uma distribuição de conexões mais adequada, possuem um coeficiente de agregação muito baixo, em oposição ao que se encontra em diversas redes complexas importantes [Watts \& Strogatz, 1998], [Watts, 1999].

Modelos de redes sem escala dão maior ênfase à representação da distribuição de conexões, enquanto os modelos small-world privilegiam a representação do menor caminho médio e do coeficiente de agregação de redes complexas. Ambos modelos têm sido aplicados com sucesso no estudo de diversas redes importantes [Watts \& Strogatz, 1998], [Watts, 1999], [Albert \& Barabási, 2002]. Os modelos de redes sem escala, entretanto, normalmente baseiam-se em redes que apresentam crescimento [Albert \& Barabási, 2002], [Wang \& Chen, 2003], isto é, redes em que o número elementos, ou nós, cresce com o tempo. Isso, para o propósito de avaliar a dinâmica de elementos que se encontram interligados, traz uma série de dificuldades. Por outro lado, modelos de rede small-world, além de representarem adequadamente uma série de redes complexas que se observam [Watts \& Strogatz, 1998], [Strogatz, 2001], [White \& Houseman, 2003], podem ser construídos a partir de redes regulares, como as encontradas em acoplamentos do tipo CML, e não requerem crescimento da rede. Prestam-se, portanto, melhor à comparação da dinâmica de seus elementos com a de modelos regulares como o GCM e CML. Dessa forma, nossa escolha recai sobre modelos de rede small-world, para os quais descrevem-se a seguir a construção de três diferentes tipos.

\subsection{Small-worlds}

Uma rede small-world é um grafo que apresenta um alto coeficiente de agregação (semelhante ao encontrado em redes regulares) e um menor caminho médio pequeno, se comparado ao número de elementos da rede ${ }^{4}$ (semelhante ao encontrado em redes aleatórias).

\footnotetext{
${ }^{4} \mathrm{O}$ conceito conhecido por "seis graus de separação", pelo qual as pessoas estariam separadas umas das outras em média por seis conhecidas, é, talvez, a manifestação mais popular dessas redes. Tal conceito tem origem no experimento do psicólogo Stanley Milgram que, em 1967, enviou centenas de cartas solicitando às pessoas para que as passassem, pessoalmente, para conhecidos próximos de forma que elas pudessem chegar a um destinatário em Boston. As cartas que chegaram passaram, em média, por seis pessoas. Para revisão histórica de redes small-world, ver [Watts, 1999].
} 
Em geral, ainda associa-se às redes small-worlds um grande número de elementos e um número pequeno de conexões por nó.

Essas redes parecem permitir uma maior velocidade na propagação de sinais e informações, maior capacidade de sincronismo e poder computacional, se comparadas às topologias regulares [Strogatz, 2001]. Há um grande número de trabalhos voltados ao estudo dessas redes em sistemas dinâmicos [Lago et al., 2000], [Barahona \& Pecora, 2001], [Wang \& Chen, 2002], [Wang \& Chen, 2002b] e redes neurais [Watts \& Strogatz, 1998], [Gade \& Hu, 2000], [Bohland \& Minai, 2001], [Moyano et al., 2001], [Barábasi, 2002], sendo a maior parte voltada para análise da evolução desses sistemas para um estado coerente (todos elementos sincronizados) e a comparação com sistemas de topologia regular. Neste trabalho, investigamos o surgimento de grupos de mapas sincronizados em tipos diferentes de redes small-world. Mais precisamente, investigamos o modelo original de WattsStrogatz (WS) e duas de suas variantes: o modelo de Newman-Watts (NW) e o modelo "smallest-world" (SW).

\section{Modelo de Watts-Strogatz, WS}
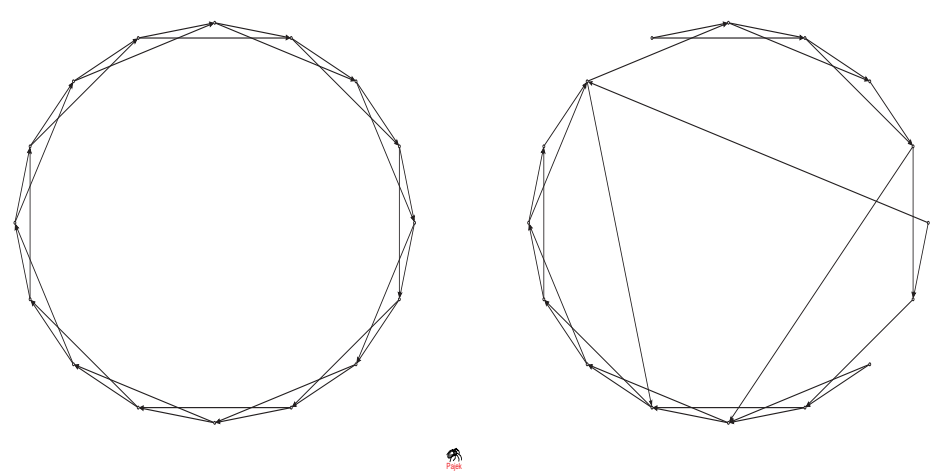

Figura 4.4: Rede regular e uma rede Watts-Strogatz (WS) produzida a partir dela.

A rede small-world de Watts-Strogatz (WS) [Strogatz, 2001] é construída a partir de uma rede de regular do tipo $k$-vizinhos mais próximos, sendo idêntica àquela empregada no acoplamento de mapas CML. Consideram-se, como anteriormente, as condições de contorno periódicas $x_{N+1}=x_{1}$, sendo $N$ o número de elementos da rede (ver seção (2.7)). A rede é, portanto, um anel. Cada conexão é, então, refeita, com probabilidade $p$, escolhendo-se aleatoriamente um novo vértice final. A probabilidade de que a conexão seja mantida vale $1-p$. Arestas duplicadas e auto-conexões não são permitidas. A probabilidade $p$ denomina-se probabilidade de religação. 


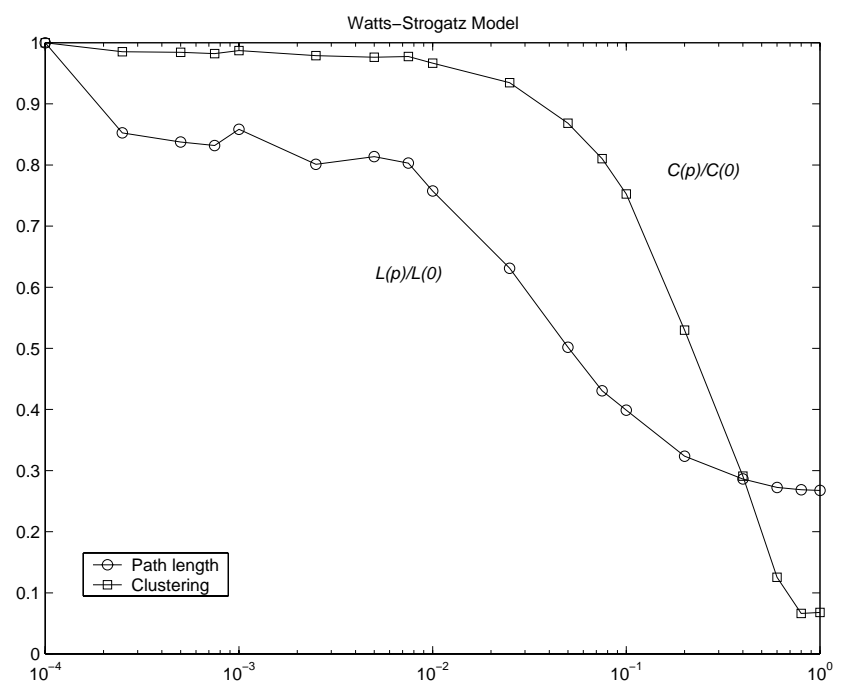

Figura 4.5: Valores de $C(p)$ e $L(p)$ para uma rede WS de 50 elementos e $k=4$, normalizados pelos valores do grafo regular, $C(0)$ e $L(0)$.

Sendo redes aleatórias, o coeficiente de agregação e o menor caminho médio da rede WS passa a ser função de $p$. $C(p)$ e $L(p)$, em geral, não apresentam uma expressão analítica para determinação de seus valores, que somente são acessíveis através de medida direta nas rede ou através de estimativas. $C(0)$ e $L(0)$ correspondem aos valores do grafo regular, enquanto $C(1)$ e $L(1)$ correspondem aos valores do grafo aleatório. Os valores $C(p)$ e $L(p)$ apresentam uma relação importante encontrada nos modelos de redes smallworld e representada na figura (4.5): o menor caminho médio decresce rapidamente com o crescimento da probabilidade $p$ enquanto o coeficiente de agregação decresce com taxa menor. Como conseqüência, modelos de small-world permitem diminuir consideravelmente o menor caminho médio de uma rede regular sem uma redução significativa da agregação local de seus elementos.

\section{Modelo de Newman-Watts, NW}

Dependendo da probabilidade $p$, o procedimento descrito anteriormente pode levar a sub-redes desconectadas. Desse modo, Newman e Watts [Newman \& Watts, 1999] propuseram um modelo no qual as novas arestas são adicionadas com uma probabilidade $p$, sem a remoção de quaisquer conexões existentes. O modelo de Strogatz-Newman pode ser adaptado adicionando-se uma regra para não permitir religações que gerem sub-redes desconectadas e adotamos, aqui, essa técnica. Note-se, entretanto, que o modelo WS preserva o número de conexões original da rede regular, enquanto no modelo de NewmanWatts (NW) a rede criada apresenta um número maior de arestas. 

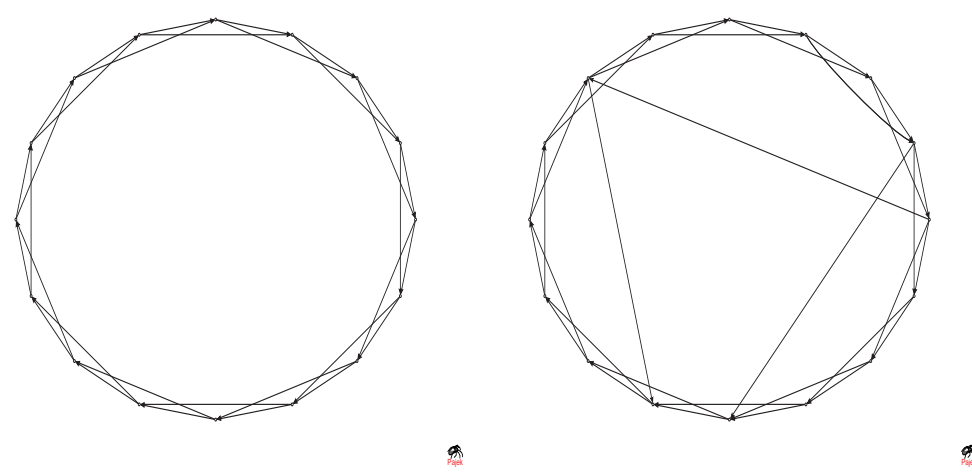

Figura 4.6: Rede regular e uma rede Newman-Watts (NW) produzida a partir dela.

\section{Modelo "smallest-world", SW}
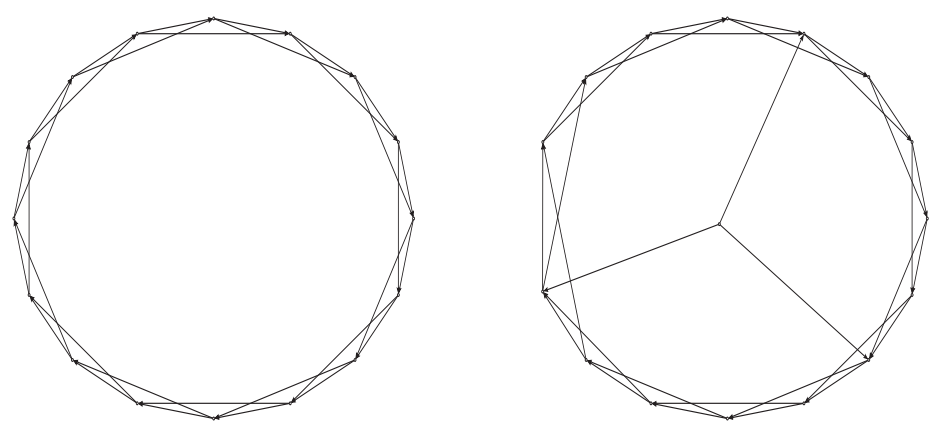

Figura 4.7: Rede regular e uma rede "smallest-world" (SW) produzida a partir dela.

Outra variante do modelo Watts-Strogatz pode ser obtida adicionando-se à rede regular alguns elementos centrais escolhidos aleatoriamente e conectados aos nós originais da rede [Newman, 2000], [Dorogovtsev \& Mendes, 2002] em uma configuração estrela. Se apenas um elemento central é adicionado, denomina-se esse modelo como smallest-world (SW) [Dorogovtsev \& Mendes, 2002].

Todos esses modelos exibem as propriedades de um "elevado" coeficiente de agregação e um menor caminho médio proporcional a $\ln (N)$.

\subsection{Mapas acoplados em redes small-world}

Partimos de mapas globalmente acoplados (GCM) adaptando-os para a construção de nosso modelo. Seja o seguinte conjunto de mapas globalmente acoplados: 


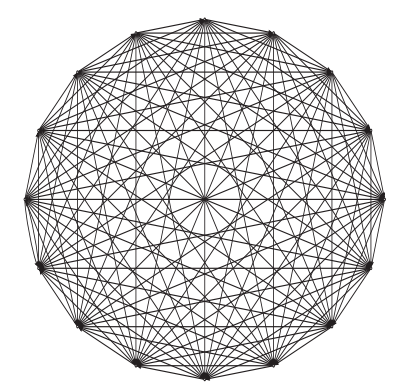

(a)

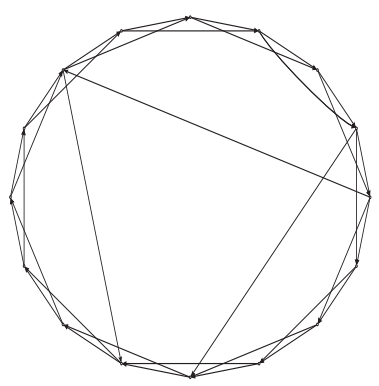

* (d)

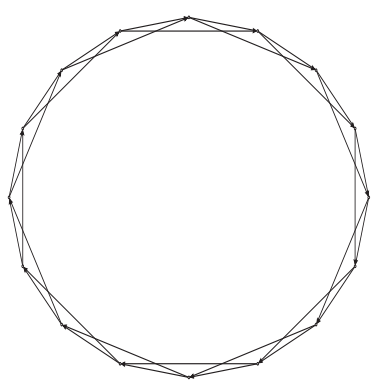

* (b)

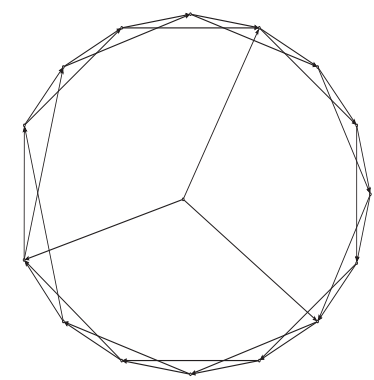

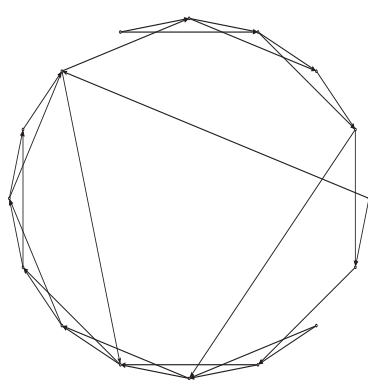

2(c)

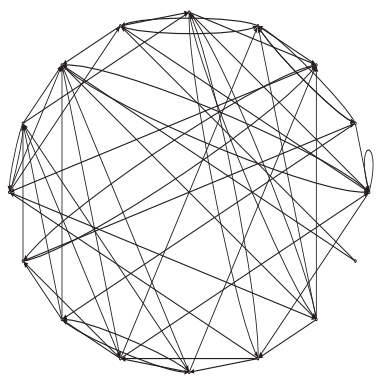

(e)

Figura 4.8: Diferentes modelos de redes. Dois modelos de conexões regulares: (a) uma rede de elementos totalmente conectados, esquema empregado no acoplamento de mapas do tipo GCM, com $N=16$ elementos; e (b) uma rede do tipo $k$-vizinhos mais próximos, empregado no acoplamento local de (CML), com $N=16$ e $k=4$. Três modelos de redes small-world construídos a partir do grafo regular (b): (c) Watts-Strogatz, WS; (d) Newman-Watts, NW; e (e) smallest-world, SW; ao final, uma rede aleatória (f). 


$$
x_{i}(t+1)=(1-\varepsilon) f_{\mu}\left(x_{i}(t)\right)+\frac{\varepsilon}{N} \sum_{i=1}^{N} f_{\mu}\left(x_{i}(t)\right)
$$

sendo $x_{i}(t)$, com $i=1, \ldots, N$, variáveis de estado que evoluem no tempo discreto $t$ e $f_{\mu}(x)$ o mapa logítico, $f_{\mu}(x)=\mu x(1-x)$, com o parâmetro de bifurcação $\mu$. O acoplamento global é dado pelo termo $\sum_{i=1}^{N} f_{\mu}\left(x_{i}(n)\right)$ e $\varepsilon$ é a constante de acoplamento do sistema.

A fim de termos uma forma única de representar sistemas GCM e mapas acoplados em diferentes topologias de rede small-world, reescrevemos a equação (4.4) incluindo a matriz $\mathbf{C}, N \times N$, de acoplamento do sistema, de modo que:

$$
\mathbf{x}(t+1)=(1-\varepsilon) f_{\mu}(\mathbf{x}(t))+\frac{\varepsilon}{N_{j}} \mathbf{C} \cdot f_{\mu}(\mathbf{x}(t))
$$

sendo agora $\mathbf{x}(t)=\left(x_{1}(t), \ldots, x_{N}(t)\right)^{T} \in \mathbf{R}^{N}$, em que $\left(x_{1}(t), \ldots, x_{N}(t)\right)^{T}$ indica a transposta da matriz linha $\left(x_{1}(t), \ldots, x_{N}(t)\right)$, as variáveis de estado e $f_{\mu}(\mathbf{x})$ uma função a valores vetoriais $f_{\mu}(\mathbf{x})=\left(f_{\mu}\left(x_{1}\right), \ldots, f_{\mu}\left(x_{N}\right)\right)^{T}$.

C, então, é uma matriz simétrica em que os elementos $c_{i j}$ são 1 , se $i$ e $j$ encontramse conectados, e 0 , caso contrário. $N_{j}$ representa o número de elementos não nulos na j-ésima coluna de $\mathbf{C}$ (número de conexões do elemento $j$ ).

Desse modo, mapas acoplados em modelos de rede regular ou small-world podem ser representados pelo sistema dinâmico discreto (4.5) com a matriz $\mathbf{C}$ referente a cada tipo de acoplamento.

- Modelo regular, CML. Considerando-se uma rede de $k$-vizinhos mais próximos, a matriz de acoplamento $\mathbf{C}$ é simétrica na qual a $i$-ésima linha apresenta os seguintes elementos não nulos $c_{i, j-(k / 2)}, \ldots, c_{i, j-1}, c_{i, j+1} \ldots, c_{i, j+(k / 2)}$.

- Modelo de Watts-Strogatz, WS. As regras de construção de uma rede WS podem ser aplicadas sobre a matriz de acoplamento $\mathbf{C}$ associando-se a cada religação $(i, j) \rightarrow$ $(i, m)$, ocorrida com probabilidade $p$, às transformações $c_{i, m}=c_{m, i}=0 \rightarrow 1$, para adicionar a nova conexão; e $c_{i, j}=c_{j, i}=1 \rightarrow 0$, a fim de remover a conexão $(i, j)^{5}$.

\footnotetext{
${ }^{5}$ Nota: aqui cada elemento da rede é representado por um único índice e $(i, j)$ representa, então, uma ligação dos elementos $i$ e $j$. Esta notação diferencia-se da empregada no capítulo 3, em que os elementos encontram-se em uma matriz e $(i, j)$, portanto, refere-se a um elemento da rede.
} 


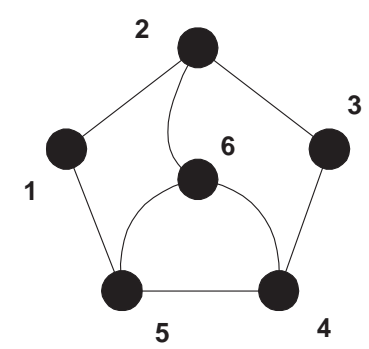

Figura 4.9: Grafo não-direcionado e sua matriz de acoplamentos na tabela (4.2).

- Modelo Newman-Watts, NW. Nesse caso, a matriz de acoplamento C é construída a partir da rede regular apenas com a adição de novas conexões $c_{i, m}=c_{m, i}=1$ para cada nó $i$, com probabilidade $p$ de que ocorram.

- Modelo smallest-world, SW. Esse modelo é construído escolhendo-se, a partir do grafo regular, um elemento arbitrário $c$ tal que $1<c<N$, que passa a ser o centro da rede. As arestas $(c, i)$ são adicionadas com uma probabilidade $p$, fazendo-se $c_{c, i}=c_{i, c}=1$.

\section{Coeficiente de agregação e menor caminho}

O grafo regular inicial e que dá origem as redes WS, NW e SW permite o cálculo dos coeficiente agregação e menor caminho médio pelas expressões [Watts, 1999]:

$$
\begin{aligned}
L(0) & =\frac{N(N+k-2)}{2 k(N-1)} \\
C(0) & =\frac{3(k-2)}{4(k-1)}
\end{aligned}
$$

Para $p \neq 0$, esses valores variam com a probabilidade $p$ e foram obtidos pela avaliação direta das redes obtidas. A figura (4.11) adiante mostra os resultados obtidos em nossas simulações numéricas. Todas as redes apresentam o efeito small-world, com um rápido decréscimo do menor caminho médio da rede a medida que $p$ aumenta, mantendo-se praticamente inalterado o coeficiente de agregação para pequenos valores de $p$.

O avaliação direta de $L$ para $N$ "grande"possui um alto custo computacional. Sendo C equivalente a matriz de adjacências de uma grafo [Lucchesi et al., 1979], adotamos um algoritmo semelhante ao de busca em grafos chamado breadth first search [Menezes, 2001]: a $i$-ésima linha de $\mathbf{C}$ fornece todos os vizinhos que distam de 1 do $i$-ésimo elemento. Os 


\begin{tabular}{c|cccccc}
$i, j$ & $\mathbf{1}$ & $\mathbf{2}$ & $\mathbf{3}$ & $\mathbf{4}$ & $\mathbf{5}$ & $\mathbf{6}$ \\
\hline $\mathbf{1}$ & 1 & 1 & 0 & 0 & 1 & 0 \\
$\mathbf{2}$ & 1 & 1 & 1 & 0 & 0 & 1 \\
$\mathbf{3}$ & 0 & 1 & 1 & 1 & 0 & 0 \\
$\mathbf{4}$ & 0 & 0 & 1 & 1 & 1 & 1 \\
$\mathbf{5}$ & 1 & 0 & 0 & 1 & 1 & 1 \\
$\mathbf{6}$ & 0 & 1 & 0 & 1 & 1 & 1
\end{tabular}

Tabela 4.2: Matriz de acoplamento $\mathbf{C}$ do grafo da figura (4.9).

\begin{tabular}{c|cccccc}
$L_{i j}, j$ & $\mathbf{1}$ & $\mathbf{2}$ & $\mathbf{3}$ & $\mathbf{4}$ & $\mathbf{5}$ & $\mathbf{6}$ \\
\hline $\mathbf{1}$ & 1 & 1 & 0 & 0 & 1 & 0 \\
$\mathbf{2}$ & 2 & 2 & 1 & 1 & 2 & 1
\end{tabular}

Tabela 4.3: Matriz para obtenção das distâncias entre nós do grafo (4.9).

elementos que distam de 2 são obtidos adicionando-se, à $i$-ésima linha, todos as linhas de elementos conectados à distância de $1\left(c_{i j}=1\right)$.

Fazemos uso ainda do fato de $\mathbf{C}$ ser simétrica $\left(L_{i j}=L_{j i}\right)$ e de que os grafos aqui são conexos. Desse modo, chegamos a uma forma suficientemente eficiente para avaliação de $L$. As tabelas (4.2) e (4.3) exibem o procedimento para um grafo de pequenas dimensões (figura 4.9).

\section{Sincronismo}

Identificamos o surgimento de estados sincronizados empregando a distância de fase $D_{i j}\left(\tau_{\ell}\right)$ entre dois elementos $i, j$ como segue. Aqui, a condição suficiente para que dois elementos estejam sincronizados é que $\left|x_{i}(t+1)-x_{j}(t+1)\right|<\delta\left|x_{i}(t)-x_{j}(t)\right|$, sendo $\delta<1$ uma constante ${ }^{6}$ [Gelover et al., 2000]. Se essa condição não é satisfeita, estados $i$ e $j$ não pertencem ao mesmo cluster, e adicionamos 1 à distância de fase de $i, j$, $D_{i j}\left(\tau_{\ell}\right)=D_{i j}\left(\tau_{\ell}\right)+1$ no tempo $\tau_{\ell}$. Se, por outro lado, a condição se verifica, os elementos encontram-se sincronizados e sua distância de fase é mantida inalterada. Uma definição semelhante de distância de fase é encontrada em [Maza et al., 2000].

Para obter a distância de fase $D_{i j}\left(\tau_{\ell}\right)$, consideramos então todos os valores $x_{i}$ e $x_{j}$ em uma janela de tempo $\ell$, isto é, os estados de $x\left(\tau_{\ell}\right)$ até $x\left(\tau_{\ell}+\ell\right)$. Desse modo, a matriz $\mathbf{D}$ no instante $\tau_{\ell}$ apresenta zeros onde os elementos $i$ e $j$ exibem estados sincronizados. A matriz $\mathbf{D}$ é, então, utilizada para identificar os clusters atratores à medida que o tempo

\footnotetext{
${ }^{6}$ Essa é uma condição mais frouxa de sincronismo do que a apresentada no capítulo 2. Espera-se que, para $t \rightarrow \infty,\left|x_{i}(t)-x_{j}(t)\right| \rightarrow 0$.
} 


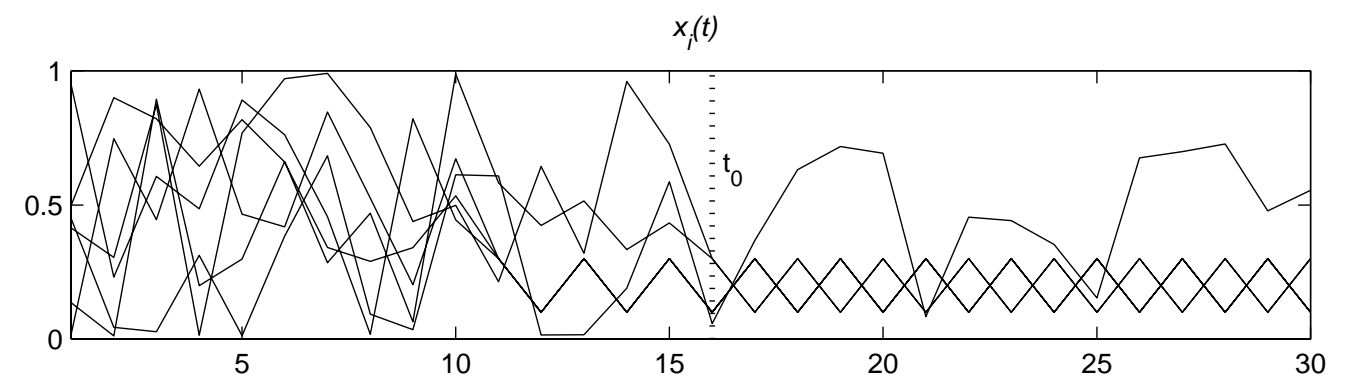

Figura 4.10: Valores de $x_{i}(t)$, para mapas acoplados na forma do grafo da figura (4.9), sincronizam a partir de um certo instante $t=t_{0}$.

\begin{tabular}{c|cccccc}
$D_{i j}(0), t<t_{0}$ & $\mathbf{1}$ & $\mathbf{2}$ & $\mathbf{3}$ & $\mathbf{4}$ & $\mathbf{5}$ & $\mathbf{6}$ \\
\hline $\mathbf{1}$ & 0 & 2 & 6 & 4 & 5 & 3 \\
$\mathbf{2}$ & & 0 & 6 & 3 & 3 & 5 \\
$\mathbf{3}$ & & & 0 & 1 & 9 & 8 \\
$\mathbf{4}$ & & & & 0 & 1 & 11 \\
$\mathbf{5}$ & & & & & 0 & 1 \\
$\mathbf{6}$ & & & & & & 0
\end{tabular}

Tabela 4.4: Matriz de distâncias de fases, em um instante $t<t_{0}$, exibe os seis elementos não-sincronizados.

\begin{tabular}{c|cccccc}
$D_{i j}(t), t>t_{0}$ & $\mathbf{1}$ & $\mathbf{2}$ & $\mathbf{3}$ & $\mathbf{4}$ & $\mathbf{5}$ & $\mathbf{6}$ \\
\hline $\mathbf{1}$ & 0 & 0 & 0 & 2 & 2 & 3 \\
$\mathbf{2}$ & & 0 & 0 & 2 & 2 & 3 \\
$\mathbf{3}$ & & & 0 & 2 & 2 & 3 \\
$\mathbf{4}$ & & & & 0 & 0 & 4 \\
$\mathbf{5}$ & & & & & 0 & 4 \\
$\mathbf{6}$ & & & & & & 0
\end{tabular}

Tabela 4.5: Matriz de distâncias de fases, em um instante $t>t_{0}$, exibe três clusters: $\{1,2,3\},\{4,5\}$ e $\{6\}$. 
evolui. As tabelas (4.4) e (4.5) mostram a matriz $D_{i j}$, para um sistema composto de seis elementos, em diferentes instantes $t$ (ver também a figura (4.10)).

Esses métodos foram empregados para análise do sincronismo de mapas acoplados em diferentes estruturas de rede small-world.

\subsection{Simulações e resultados}

Nossas simulações partem de uma rede mapas acoplados em uma topologia regular do tipo CML, com $k$ vizinhos mais próximos, e com 50 elementos. Esse número parece suficiente para considerações sobre a dinâmica de sistemas estendidos [Boccaletti et al., 2002] e é aqui empregado. No modelo, cada vértice é conectado aos seus $k=4$ vizinhos mais próximos satisfazendo $N \gg k>\ln (N)$. Procuramos, desse modo, criar o efeito smallworld sobre na topologia da rede.

Nas redes consideradas (WS, NW e SW) é mantido o número de elementos, $N=50$. Isso significa que, em nenhuma das reconexões efetuadas, vértices da rede são adicionados ou removidos. Nas religações, sub-redes com elementos desconectados também não são permitidas, sendo, nesse caso, reiniciado um novo rearranjo. Também não são consideradas religações que não alteram a original e que, portanto, sempre podem ser obtidas fazendo-se $p=0$.

\section{Menor caminho e agregação}

A figura (4.11) mostra como o menor caminho médio, $L(p)$, decresce rapidamente com a probabilidade $p$ para os diferentes modelos de redes small-world (WS, NW e SW) com pequena alteração do coeficiente de agregação $C(p)$ para baixos valores de $p$. Nesses modelos, isso equivale a dizer que se podem obter caminhos médios pequenos mantendose um número de conexões ainda da ordem $N, \mathcal{O}(N) \sim(2 k-1) N$, como ocorre nas redes regulares, em forte contraste com as $\mathcal{O}\left(N^{2}\right)$ conexões das redes globalmente acopladas $(\operatorname{com} N(N-1) / 2$ arestas $)$. 

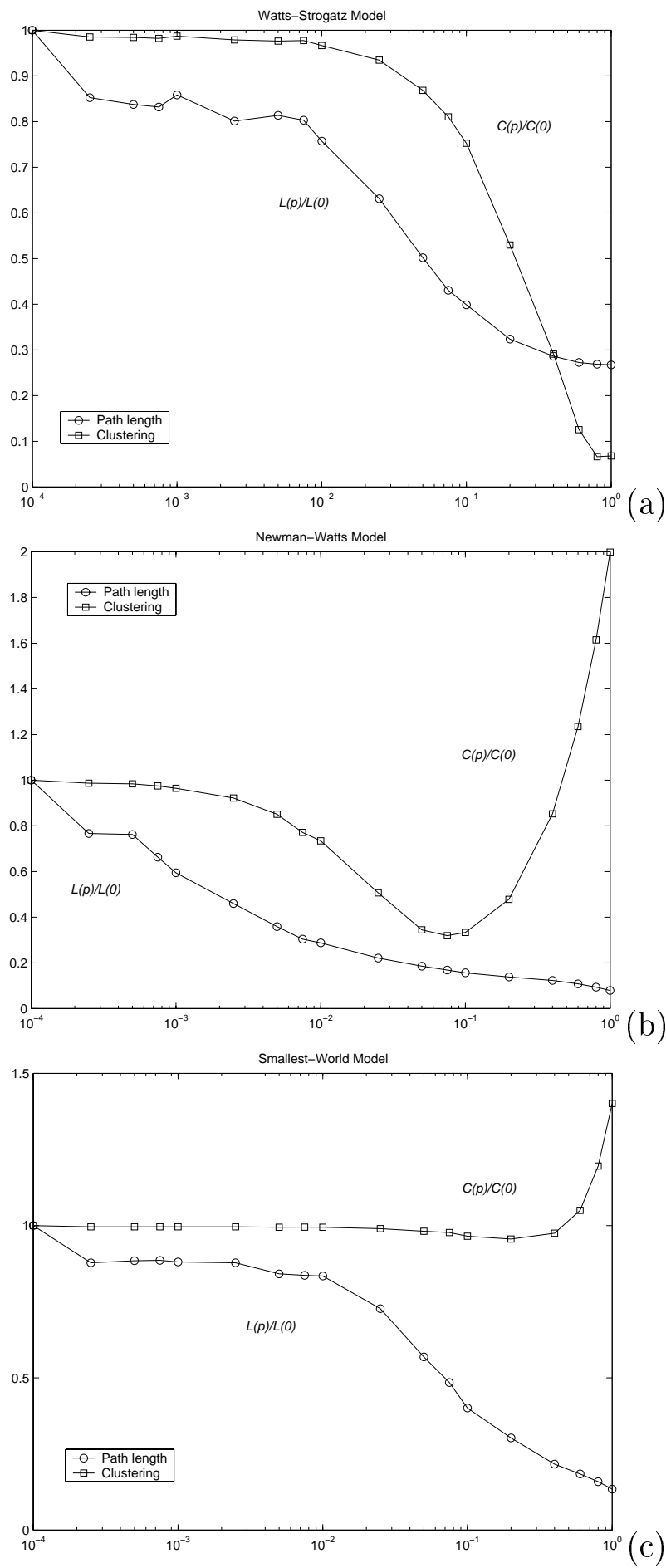

Figura 4.11: O menor caminho médio e o coeficiente de agregação normalizados pelos valores da rede regular, $L(p) / L(0)$ e $C(p) / C(0)$, para os diferentes modelos de redes small-world: (a) rede WS; (b) rede NW; e (c) rede SW. Resultados obtidos a partir de 20 realizações aleatórias de religações sobre uma rede do tipo $k$-vizinhos mais próximos, com $N=50$ e $k=4$. A probabilidade de religação é exibida em escala logarítmica. 

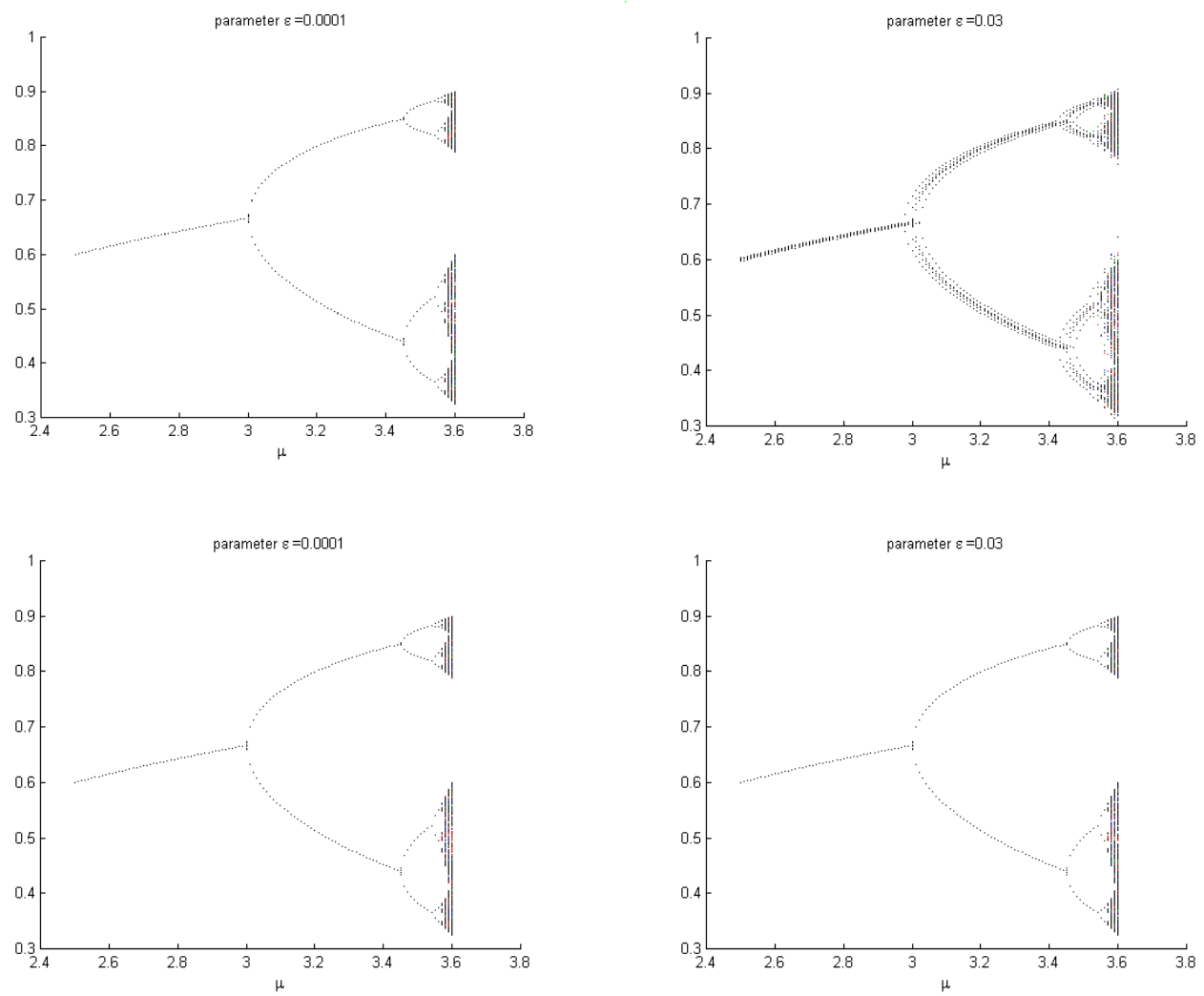

Figura 4.12: Diagramas de bifurcação para mapas acoplados em uma topologia de rede small-world WS (acima) e acoplados em uma topologia de rede regular (CML). Os dados foram obtidos para uma configuração de $N=50$ e $k=4$, computados após 2000 iterações, e exibidos os 50 últimos estados de cada sistema. Os parâmetros de acoplamento utilizados foram $\varepsilon=0.0001$ e $\varepsilon=0.03$. Observa-se que para valores maiores de $\varepsilon$, o sistema WS apresenta um maior número de clusters (ver detalhe na figura (4.13)), enquanto no sistema regular o número de clusters permanece inalterado.

\section{Sincronismo e bifurcações}

Se uma rede regular do tipo CML tem um número suficientemente grande de elementos, o surgimento do estado coerente, com todos os elementos completamente sincronizados, é praticamente impossível de ser obtido [Wang \& Chen, 2002b], [Wang \& Chen, 2003]. Entretanto, a formação de um pequeno número de clusters é relativamente fácil de ser obtida com o incremento da constante de acoplamento do sistema. Nas topologias smallworld, entretanto, valores maiores de $\varepsilon$ favorecem o surgimento de novos clusters, como pode ser observado nas figuras (4.12), (4.13) e (4.14), com o alargamento das faixas do gráfico de bifurcação. Uma vez que estamos interessados em estados que oscilam, e no sincronismo dessas órbitas em clusters em número superior a um e menor que $N$, escolhemos os parâmetros $\mu=3.45$ e $\varepsilon=0.0001$ de modo a obter esses comportamentos. 


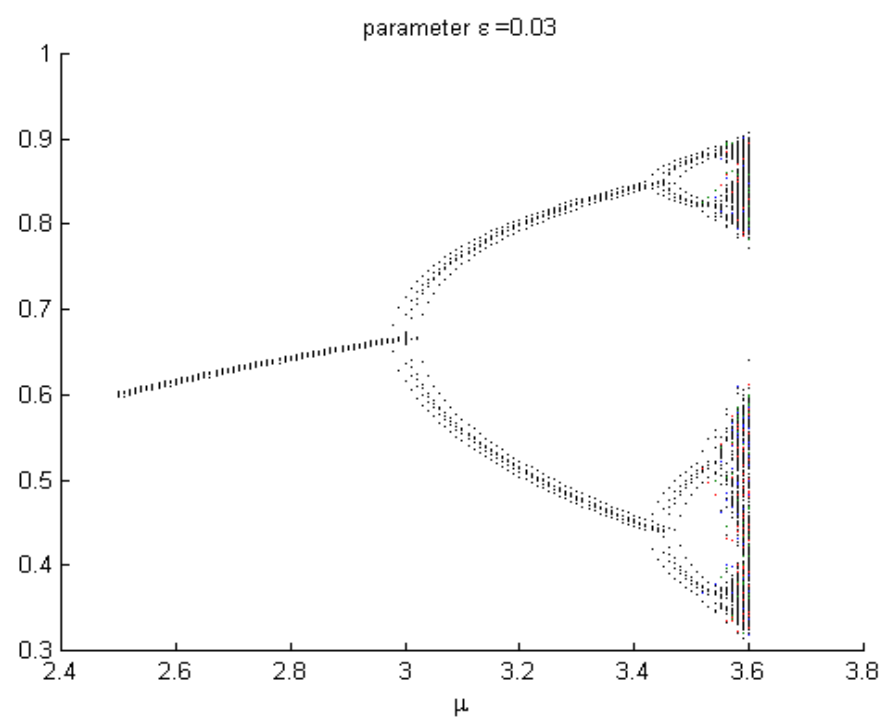

Figura 4.13: Detalhe da figura 4.12. Faixas largas indicam a presença de um maior número de clusters na rede WS.

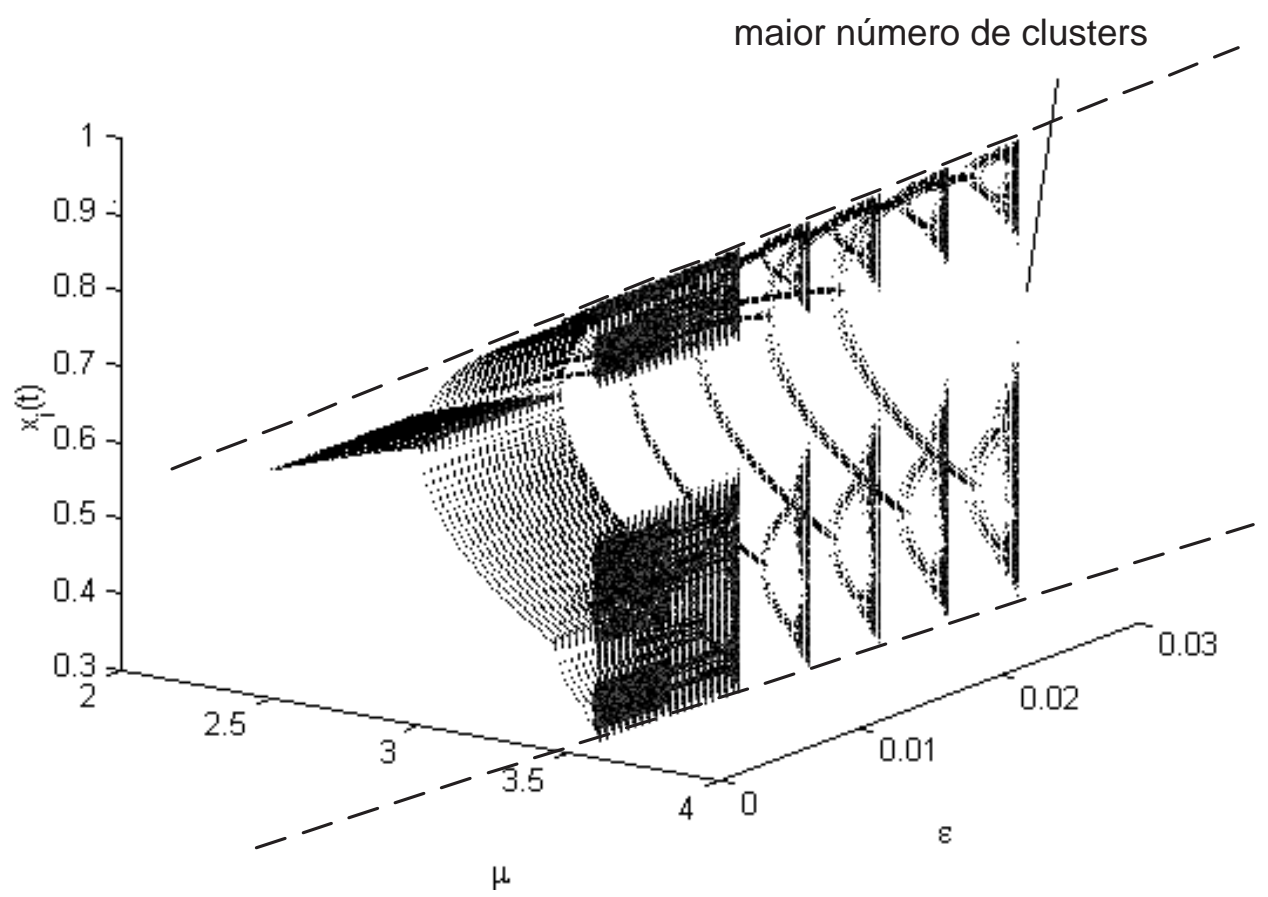

Figura 4.14: Diagrama de bifurcação a dois parâmetros destacando o papel do parâmetro $\varepsilon$ na formação de um maior número de clusters à medida que $\varepsilon$ cresce. 


\section{Clusters}

O surgimento de clusters é observado através da evolução de $\mathbf{D}\left(\tau_{\ell}\right)$. Para cada instante $\tau_{\ell}$, calculamos a matriz $\mathbf{D}\left(\tau_{\ell}\right)$ com uma janela de tempo $\ell=20$. Se $D_{i j}=0$ no instante $\tau_{\ell}$, os elementos $i$ e $j$ encontram-se sincronizados e pertencem, portanto, ao mesmo cluster. Podemos, então, obter o número de clusters diferentes a cada instante. A figura (4.15) mostra como o número de clusters evolui para acoplamentos em redes small-world WS, NW e SW com diferentes valores da probabilidade $p$.

Nossas simulações mostram que mapas têm comportamentos quantitativamente diferentes quando acoplados em topologias de redes regulares e em redes small-world. Para diferentes valores de $p$, após os estados transientes, todos os modelos de small-world (WS, NW e SW) exibem um número consideravelmente maior de clusters que no modelo regular de $k$-vizinhos mais próximos (CML) - mesmo que na reconexão uma única aresta fosse adicionada. Em particular, valores maiores do parâmetro de acoplamento favorecem o surgimento de novos clusters, e proporcionam, portanto, um estado menos coerente da rede. E, de fato, um grande número de clusters parece surgir com um incremento mínimo no valor de $\varepsilon$. O sincronismo exibe forte dependência da probabilidade $p$.

Comparando-se a dinâmica entre os diferentes tipos de acoplamento small-world (WS, NW e SW), observamos que redes com acoplamento SW exibiram a formação de um menor número de clusters para todas as probabilidades $p$, bastante próximo do encontrado na rede regular $(p=0)$. Conjecturamos, aqui, que isso se deve à presença de um elemento mais central na rede que fornece uma certa coordenação global do acoplamento ${ }^{7}$. As redes WS apresentaram números de clusters próximos aos encontrados na rede regular $(p=0)$ somente para baixos valores de $p, p<0.001$, e parecem exibir quantidades de clusters para toda faixa de valores entre 1 e $N$. As redes SW apresentaram números de clusters próximos aos encontrados na rede regular $(p=0)$ somente para baixos valores de $p, p<0.01$, e parecem exibir quantidades de clusters para toda faixa de valores entre 1 e $N$. Por último, os sistemas com conexões do tipo $N W$ exibiram um pequeno número de clusters somente para valores muito pequenos de $p, p<0.001$, nos quais as redes regulares se mostram muito pouco alteradas, exibindo um número de clusters próximo ou igual a $N$ (um estado desordenado ou totalmente assíncrono) para a maior parte dos valores de $p$. A figura (4.16) mostra esses resultados para os seguintes valores de $p=$

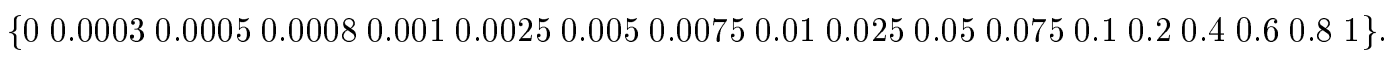

\footnotetext{
${ }^{7} \mathrm{Em}$ redes com acoplamento local, o sincronismo parece exigir uma coordenação global [Wang \& Chen, 2002], [Wang \& Chen, 2003].
} 

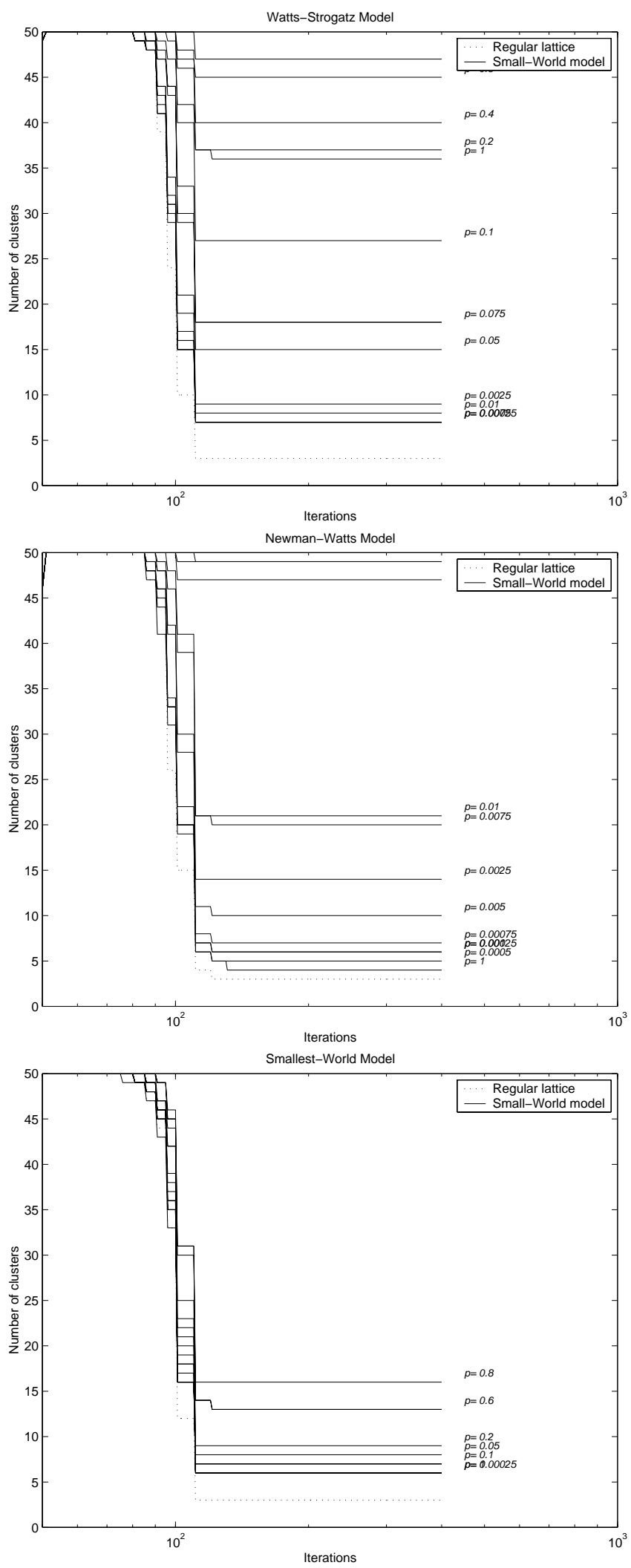

Figura 4.15: Mapas acoplados em diferentes topologias de redes small-world exibem diferentes números de clusters após o transiente da rede. As simulações foram realizadas partindo-se de uma rede regular de parâmetros $N=50$ e $k=4$. Para os parâmetros $\mu=3.45$ e $\varepsilon=0.0001$ a rede regular atinge um estado final com a formação de dois grupos de elementos - linhas pontilhadas no gráfico. A evolução do número de clusters é obtida através da matriz de distâncias de fase $D_{i j}\left(\tau_{\ell}\right)$, e exibe uma forte dependência com a probabilidade $p$. 
Evolução do número de clusters para diferentes probabilidades de religação em redes WS, NW, e SW
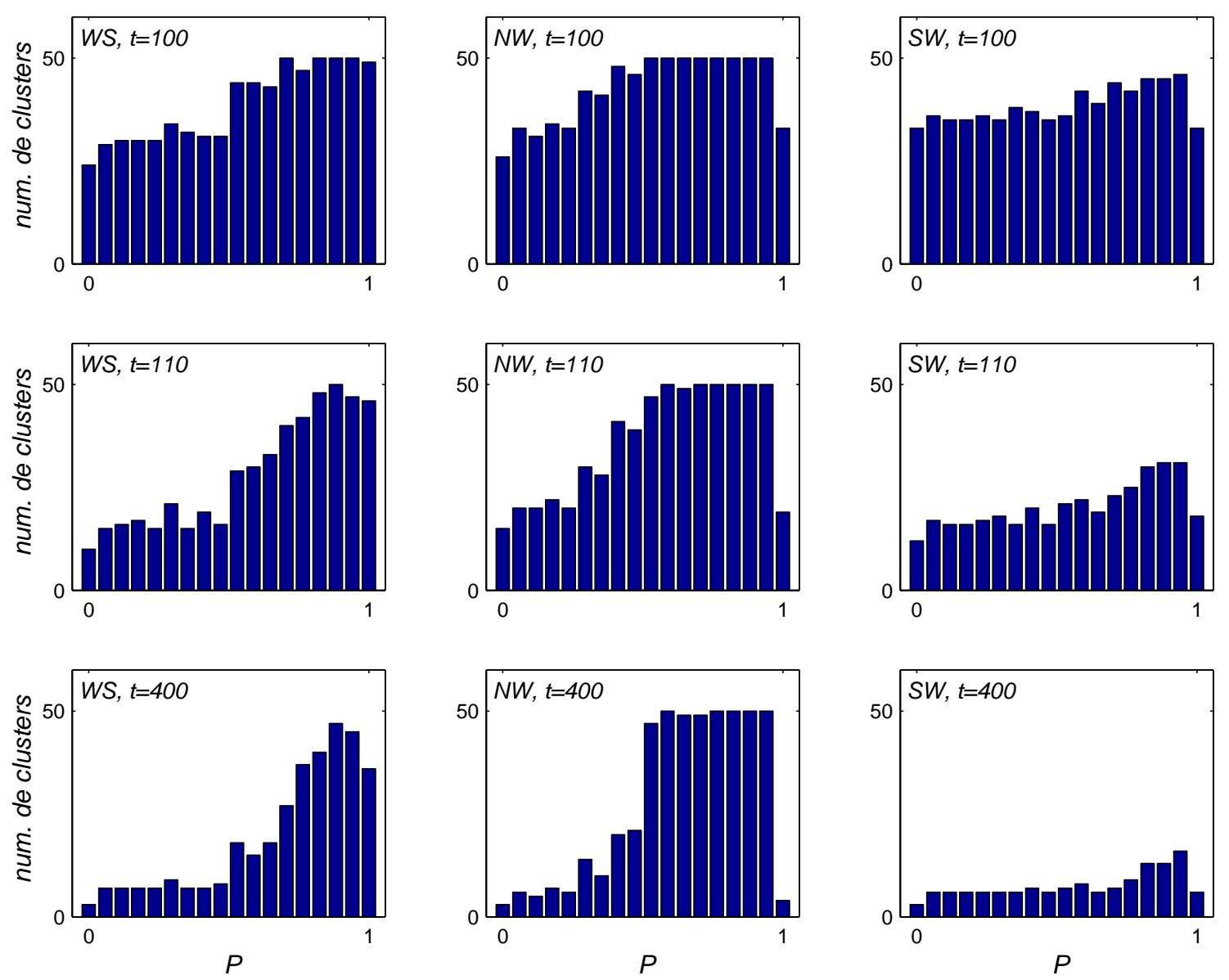

Figura 4.16: Evolução do número de clusters nos tempos $t=100,110$ e 400 para os diferentes tipos de rede WS, NW e SW, tendo sido empregados os valores de $p=$ $\{0,0.0003,0.0005,0.0008,0.001,0.0025,0.005,0.0075,0.01,0.025,0.05,0.075,0.1,0.2,0.4,0.6,0.8,1\}$.

Dessas observações, decorrem nossos principais resultados neste capítulo. Para os sistemas de mapas aqui considerados, os acoplamentos em forma de small-world parecem levar a dinâmica das redes para estados menos ordenados (número de clusters maior) quando empregamos uma arquitetura NW e para estados mais ordenados em redes do tipo SW. Já as redes WS exibem valores intermediários. Particularmente, as redes WS e SW permitem a formação de um número de clusters de ordem muito semelhante ao número obtido na rede regular, mesmo para probabilidades de religação muito baixas, o que permite a construção de modelos com esse comportamento, mas com uma menor distância média entre nós bastante inferior à encontrada nas redes regulares. 


\subsection{Conclusão e sumário}

Neste capítulo, destacamos que redes surgem em uma série de sistemas. Muitas dessas redes são complexas, com um grande número de elementos e quantidade de conexões distribuídas de um modo não uniforme. Sobre uma série de aspectos, então, essas redes não encontram uma representação adequada em modelos regulares de rede, como as utilizadas nos acoplamentos do tipo CML e GCM do capítulo 3. Dois modelos recentes de rede parecem representar, de modo mais adequado, essas redes complexas: redes smallworld e redes sem escala. Aqui, nossa escolha foi pelo uso de redes small-world, as quais julgamos mais adequadas para o estudo da dinâmica de seus elementos e para comparações com os modelos regulares. Três tipos de redes small-world foram empregados para realizarmos acoplamentos entre mapas e uma série de métodos foram empregados na análise da dinâmica dessas redes. Por fim, nosso principal resultado neste capítulo é o de que podemos empregar redes WS e SW para formação de agrupamentos (clusters) síncronos, sugerindo uma auto-organização, da mesma forma que obtemos em acoplamentos do tipo CML e GCM, mas com as seguintes vantagens: exibem um número de conexões de ordem $\mathcal{O}(N) \sim(2 k-1) N$ próximo do número das redes do tipo CML, e muito menor que o encontrado nos acoplamento do tipo GCM $\left(\mathcal{O}\left(N^{2}\right)\right)$; ao mesmo tempo, apresentam menor caminho médio bastante inferior ao dos acoplamentos do tipo CML (rápido decréscimo de $L(p)$ ). Parece-nos, então, adequado propor a utilização desses modelos (WS e SW) em aplicações que normalmente fazem uso de sistemas acoplados em topologias regulares, como sistemas para detecções de contorno, segmentação de imagens e memórias associativas. Essas aplicações e estudos mais detalhados das propriedades dinâmicas dessas redes, incluindo redes small-world de malhas de duas dimensões, deverão ser objeto de estudo futuro dos autores. 


\section{Computabilidade e processamento simbólico}

The primary source of all mathematics are the integers.

Herman Minkowski

Este penúltimo capítulo trata da capacidade de mapas serem capazes de realizar processamento simbólico. Mais precisamente, trata da capacidade de, dado um conjunto de símbolos, representados aqui, em geral, por cadeias de "0"s (zeros) e "1"s (uns), de extraírem informações sobre essa cadeia de modo análogo ao processamento realizado por programas de computador. Faz-se, inicialmente, uma revisão das principais abordagens sobre computabilidade através de mapas, partindo da composição de sistemas de portas lógicas até uso de sistemas dinâmicos em tempo discreto, propondo-se, ao final, uma aplicação de tratamento simbólico para imagens.

A capacidade de mapas acoplados e sistemas análogos resolverem quaisquer problemas que possam ser solucionados por computadores apresenta diversas abordagens na literatura [Siegelmann \& Sontag, 1991], [Koiran et al., 1994], [Orponen \& Matamala, 1996], [Moore, 1990], [Haykin, 1999], [Sato et al., 2000] e adquire importância crescente à medida que se buscam novos modos de computação [Gramß et al., 1998]. A equivalência de sistemas de mapas às máquinas computacionais requer uma série de conhecimentos como: o conceito de máquina de Turing e o de computação universal. Tais conceitos são introduzidos a seguir. 


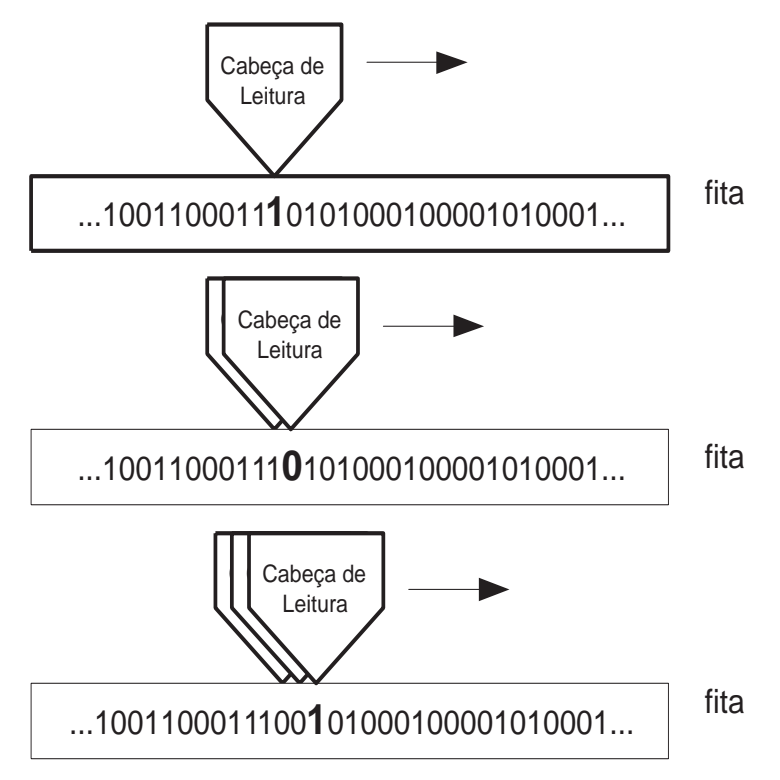

Figura 5.1: Máquina de Turing determinista.

\subsection{Máquina de Turing}

Uma máquina de Turing determinista ${ }^{1}$ [Lucchesi et al., 1979] [Minsky, 1967] é um sistema formal que pode ser visualizado como uma máquina computacional consistindo de um controle central com um conjunto finito de estados $Q$ que opera sobre uma fita de entrada/trabalho/saída (ver figura (5.1)).

A fita unidimensional encontra-se dividida em células indexadas por um inteiro $i$, $-\infty<i<+\infty$. Cada célula contém um único símbolo de um alfabeto finito $\Sigma$. A cada instante, a cabeça de leitura do controle central posiciona-se sobre uma única célula e, dependendo de seu estado interno, executa as seguintes operações:

1. Substitui o símbolo $s \in \Sigma$ por um novo símbolo $s^{\prime} \in \Sigma$.

2. Reposiciona a cabeça de leitura sobre uma célula adjacente, movendo a fita à direita $(d=-1)$ ou à esquerda $(d=+1)$.

3. Troca o seu estado interno $q \in Q$ para o estado $q^{\prime} \in Q$.

\footnotetext{
${ }^{1}$ Uma máquina de Turing não determinista possui uma fita adicional que pode ser somente lida, mas não pode ser gravada. Ver [Lucchesi et al., 1979] e [Minsky, 1967] para definições sobre outros tipos de máquina de Turing.
} 


\begin{tabular}{ccccc}
$q_{i}$ & $s_{j}$ & $s_{i j}$ & $d_{i j}$ & $q_{i j}$ \\
\hline 0 & 0 & 0 & -1 & 0 \\
0 & 1 & 1 & -1 & 1 \\
1 & 0 & 0 & -1 & 1 \\
1 & 1 & 1 & -1 & 0
\end{tabular}

Tabela 5.1: Tuplas da máquina de Turing para um verificador de paridade.

Desse modo, instruções para uma máquina de Turing são quíntuplas da forma:

(estado anterior, símbolo anterior, símbolo novo, direção da fita, estado novo)

determinadas unicamente pelo estado interno e o símbolo sobre o qual se encontra posicionada a cabeça de leitura da máquina:

$$
\left(q_{i}, s_{j}, S\left(q_{i}, s_{j}\right), D\left(q_{i}, s_{j}\right), G\left(q_{i}, s_{j}\right)\right)
$$

$\mathrm{Ou}$

$$
\left(q_{i}, s_{j}, s_{i j}, d_{i j}, q_{i j}\right)
$$

sendo $q_{i} \in Q, s_{i} \in \Sigma$ e $d_{i j} \in\{-1,0,1\}$, não havendo tuplas que coincidam nas duas primeiras entradas (condição de consistência da máquina de Turing). Em geral, adotamse um estado incial $q_{0}$ e dois estados finais $q_{a}$ e $q_{r}$ possíveis correspondentes aos estados aceita a entrada e rejeita a entrada. A operação da máquina de Turing sobre a fita encerra-se (halt) quando atinge-se um dos estados finais. A entrada não é aceita, nem rejeitada, se nenhum estado final da máquina é atingido. Nesse caso, a máquina não pára.

Embora a máquina de Turing seja mais geral, aqui consideram-se máquinas em que $\Sigma=\{0,1\}$, a exemplo dos computadores digitais. A condição de que também os estados possíveis são binários, $Q=\{0,1\}$, é restritiva, mas será útil na fomalização de algoritmos mais simples utilizados adiante.

Verificador de paridade. Esse algoritmo determina a paridade de símbolos $\{1\}$ dada uma cadeia de símbolos $s_{0} s_{1}, \ldots, s_{n}$ tal que $s_{i} \in\{0,1\}$. As tuplas que definem esse algoritmo em uma máquina de Turing podem encontradas na tabela (5.1).

A paridade é fornecida pelo estado final da máquina no qual $q=0$ significa um número par de símbolos $\{1\}$. Evita-se a necessidade de símbolos adicionais que indiquem o fim da 


\begin{tabular}{c|cc}
$q$ & $w_{1}$ & $w_{2}$ \\
\hline 0 & 1011 & \\
1 & 011 & 1 \\
0 & 11 & 10 \\
0 & 1 & 101 \\
1 & & 1101
\end{tabular}

Tabela 5.2: Configurações da máquina de Turing para o verificador de paridade com entrada 1011.

computação, assumindo-se uma fita com um número finito de elementos. O processamento não altera os símbolos da fita $\left(s_{j}=s_{i j}\right.$ em qualquer tupla) e a fita desloca-se unicamente para direita $\left(d_{i j}=-1\right)$.

Um conceito importante e utilizado adiante é o de configuração de uma máquina Turing [Sato et al., 2000]. Qualquer fita de uma máquina de Turing pode ser descrita por duas seqüências infinitas de símbolos de $\Sigma$, as palavras infinitas $w_{1}$ e $w_{2}$, sendo que $w_{1}$ representa a seqüência de símbolos sobre e à direita da cabeça de leitura e $w_{2}$ a seqüência de símbolos à esquerda da cabeça de leitura. O conjunto das tuplas $\left(q_{i}, w_{1, i}, w_{2, i}\right)$ constituise, então, em um conjunto de configurações da máquina de Turing. Desse modo, se o índice $i=0$ indica a posição da cabeça de leitura sobre a fita $\ldots s_{-2} s_{-1} s_{0} s_{1} s_{2} \ldots$ então $w_{1}=s_{0} s_{1} s_{2}\left(s_{3}\right) \ldots$ e $w_{2}=s_{-1} s_{-2}\left(s_{-3}\right)$ de modo que (.) denota a repetição indefinida do símbolo. Assim:

$$
\ldots s_{-2} s_{-1} s_{0} s_{1} s_{2} \ldots \Longleftrightarrow w_{1}=s_{0} s_{1} s_{2}\left(s_{3}\right) \text { e } w_{2}=s_{-1} s_{-2}\left(s_{-3}\right)
$$

Verificador de paridade. A tabela (5.1) mostra o conjunto de configurações de uma máquina de Turing para o algoritmo de verificação de paridade aplicado, por simplicidade, a uma fita finita constituída dos elementos 1011. O estado final da máquina é, como esperado, $q=1$ (número ímpar de $\{1\}$ ).

\subsection{Computação universal}

Uma das idéias centrais da teoria da computação é o da equivalência de diferentes modelos formais de algoritmos. A máquina de Turing é, provavelmente, o modelo formal de algoritmos mais empregado na teoria da computação [Lucchesi et al., 1979]. Há outros modelos, entretanto, que apresentam poder computacional equivalente à máquina de Turing, baseados em outros formalismos. Dentre eles, os mais freqüentemente encontra- 
dos estão os modelos de funções recursivas (Gödel \& Herband), máquinas baseadas em registradores (Shepherdson \& Sturgis), o $\lambda$-Cálculo (Church \& Kleene), gramáticas estruturadas (Chomsky), cadeias de Markov (Markov) e sistemas de regravação (Post). Todos compartilham uma série de propriedades com as máquinas de Turing: $(i)$ apresentam um conjunto preciso de instruções descrito por um conjunto finito de símbolos; (ii) produzem um resultado em um número finito de passos; ( $i i i$ ) não requerem máquina alguma para sua realização, podendo ser executados por qualquer pessoa dispondo apenas papel e lápis ${ }^{2}$; (iv) e suas realizações não requerem inteligência alguma adicional, além da necessária para a execução das instruções. Diversos textos tratam desses diferentes modelos e aqui basta considerar a existência de outros modelos equivalentes à máquina de Turing. Para uma revisão e referências sobre outros modelos ver [Hopcroft \& Ullman, 1979]. Outros sistemas formais menos convencionais, ou que não têm sua origem propriamente no processamento simbólico, também apresentam capacidade equivalente à máquina de Turing, como: os automatos celulares [Wolfram, 2001] e as redes neurais [Siegelmann \& Sontag, 1991].

A proposição de que qualquer procedimento efetivo ${ }^{3}$ pode ser realizado por uma máquina de Turing é conhecida como tese de Church-Turing ${ }^{4}$ [Lucchesi et al., 1979] [Minsky, 1967]. Desse modo, demonstrada a equivalência de um modelo à máquina de Turing, implica a capacidade do modelo de realizar qualquer procedimento efetivo ou computação.

A computação universal consiste na capacidade de um modelo computacional emular a si próprio. A máquina de Turing é, ela mesma, um procedimento efetivo e, desse modo, é possível a construção de uma máquina que implementa as funções (quíntuplas) de: busca de quíntupla arbitrária, troca de estado, leitura, gravação e movimento; e que, desse modo, possa ler quíntuplas de uma máquina de Turing arbitrária [Minsky, 1967]. Isso pode ser feito com diferentes combinações de quantidades de símbolos e estados. Minsky [Minsky, 1967] propôs uma máquina de Turing universal construída com o número reduzido de 7 estados e 4 símbolos, chamada de $\operatorname{MTU}(7,4)$; esse fato é usado mais adiante. Outros modelos, como os de Rogozhin (ver [Saito \& Kaneko, 2001]) implementam máquinas de Turing com outras quantidades de estados e símbolos $(\operatorname{MTU}(24,2)$ e MTU(10,3)).

\footnotetext{
${ }^{2}$ Embora pouco formal, essa é uma qualidade importante de todos esses modelos e é freqüentemente assim descrita.

${ }^{3}$ Não há qualquer definição formal de procedimento efetivo. Entretanto, diversas definições na teoria da computação empregam esse termo, sendo um conceito informal de programa no sentido computacional [Lucchesi et al., 1979].

${ }^{4}$ Sendo procedimento efetivo um conceito informal, a tese de Church-Turing afirma a equivalência de um conceito informal a de um conceito formal, o que não é, portanto, passível de demonstração [Lucchesi et al., 1979]. Entretanto a tese de Church-Turing é amplamente aceita.
} 

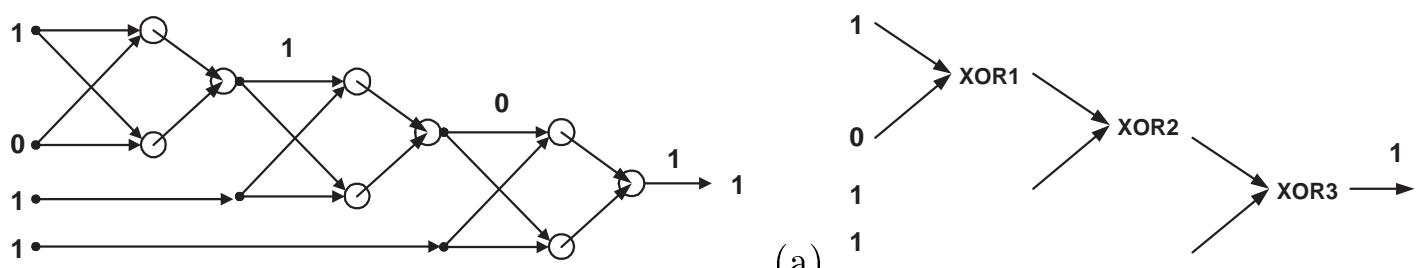

(a)

Figura 5.2: Quaisquer circuitos de portas lógicas podem ser contruídos através de um arranjo, em princípio ilimitado, de neurônios de McCulloch-Pitts, reproduzindo as funções booleanas (AND, XOR, NOT). Acima, utilizam-se três elementos que implementam a função XOR, todos distintos e conectados em série, para solução do problema da paridade do conjunto de bits 1011. Cada operador XOR na figura (b) requer três neurônios que são representados na figura (a) por circunferências.

\subsection{Computabilidade por sistemas de portas lógicas}

Desde os modelos neurais de McCulloch-Pitts (ver [Haykin, 1999]), são conhecidas formas de se construir máquinas de estados finitos através de redes desses elementos: como eles podem reproduzir quaisquer funções booleanas (AND, XOR, NOT), qualquer circuito lógico pode ser construído por uma rede desses elementos e emular uma máquina de Turing, tendo-se, em princípio, um número ilimitado de elementos [Siegelmann \& Sontag, 1991], [Bar-Yam, 1997] (ver figura (5.2)).

Dessa forma, funções simples capazes de implementar operações binárias podem ser organizadas de modo a constituirem uma rede que realiza qualquer tipo de computação [Bar-Yam, 1997]. Apresentada desse modo, a capacidade computacional dos mapas é evidente (considere um único passo de computação), mas sua aplicação sofre fortes limitações, seja pela necessidade de um número arbitrário de elementos, seja pelo fato de uma nova composição dos elementos ser requerida para cada procedimento a ser simulado [Bar-Yam, 1997].

A necessidade de um número, em princípio, arbitrário de elementos, constitui-se em uma forte limitação dessa alternativa que pode ser eliminada adicionando-se algum tipo de memória a essa rede. Isso pode ser feito introduzindo-se algum mecanismo de recorrência (feedback) na rede [Haykin, 1999], [Oliveira, 2000]. A figura (5.3) ilustra como a adição de memória através de elementos recorrentes possibilita reduzir o número de elementos na execução de um procedimento. 


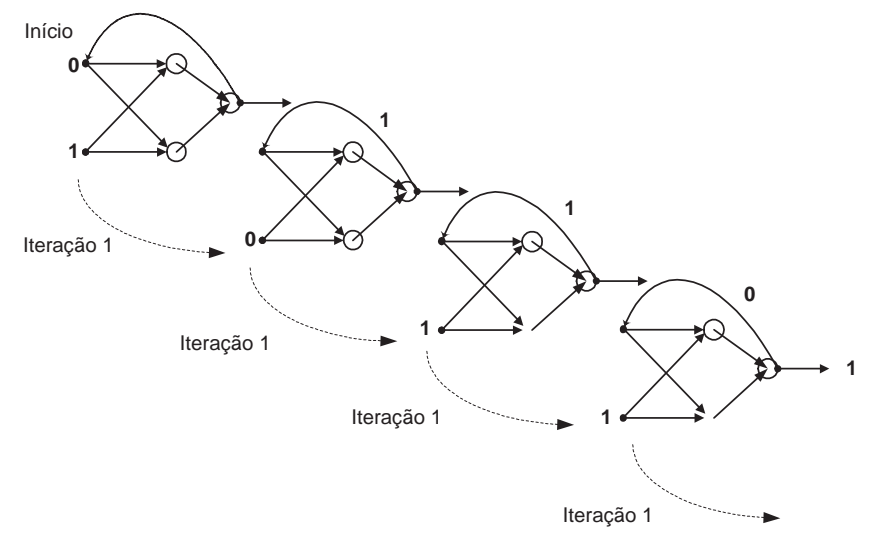

(a)

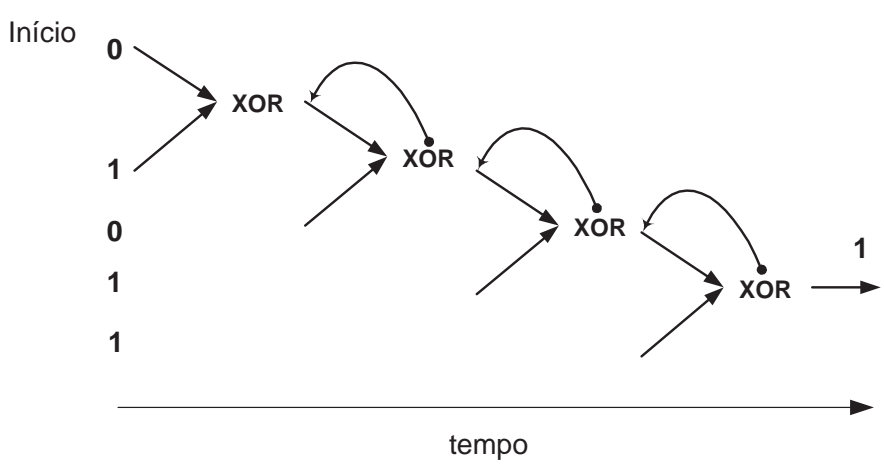

(b)

Figura 5.3: A inclusão de recorrência adiciona memória ao sistema e permite a redução do número de elementos na execução de um procedimento computacional. Acima, empregase um único elemento que realiza a função XOR para solução do problema da paridade do conjunto de bits 1011. A figura (b) simplifica a representação de (a). 
Neurônios de McCulloch-Pitts são representados por:

$$
y_{k}=\sigma\left(\sum_{j=1}^{n} w_{k j} x_{j}+b_{k}\right)
$$

sendo $x_{1}, \ldots, x_{n}$ são as entradas, $w_{k 1}, \ldots, w_{k n}$ os pesos sinápticos associados a cada entrada no neurônio $k$ e $b_{k}$ é denominado o bias ou limiar do neurônio $k$. $\sigma(x)$ é a função de ativação. Se $\sigma(x)$ é a função de Heaviside (=1 se $x \geq 0$ e $=0$ se $x<0$ ), tem-se o neurônio original de McCulloch-Pitts ${ }^{5}$ sendo comum também funções de ativação do tipo sigmóide ou linear [Haykin, 1999], [Oliveira, 2000].

Redes recorrentes desses elementos podem, então, ser reescritas na forma do mapa [Siegelmann \& Sontag, 1991], [Sontag, 1996]:

$$
x(t+1)=f(x)=\sigma(A x(t)+b u(t)+c)
$$

sendo $\sigma(x)$ a função de ativação a valores vetoriais $\sigma(x)=\left(\sigma\left(x_{1}\right), \ldots, \sigma\left(x_{n}\right)\right)$ e $A \in \mathbf{R}^{n} \times \mathbf{R}^{n}$ a matriz de pesos sinápticos dos $n$ neurônios (mapas) da rede. O vetor $u(t) \in \mathbf{R}^{n}$ é interpretado como entradas externas e $b \in \mathbf{R}$ é um valor escalar (ver também a seção (2.7) sobre a equivalência de sistemas neurais recorrentes e sistemas de mapas estendidos).

O uso de sistemas neurais recorrentes, formalizados como o sistema de mapas (5.6), permitiram a Siegelmann [Siegelmann \& Sontag, 1991] mostrar que um número limitado de elementos recorrentes como esses pode simular qualquer procedimento efetivo. Embora seja um resultado bastante importante, o número encontrado por Siegelmann foi bastante elevado $\left(\sim 10^{5}\right)$. Alguns estudos que se seguiram demonstraram a possibilidade do uso desses elementos, em um número bastante menor. No que segue, considera-se o uso de mapas lineares em intervalos para computação.

\footnotetext{
${ }^{5}$ Ver [Haykin, 1999] para as implementações das funções AND, XOR e NOT através de neurônios articiais de McCulloch-Pitts.
} 


\subsection{Máquinas de Turing e mapas lineares em inter- valos}

A abordagem, a seguir, mostra a equivalência entre mapas lineares $f: \mathbf{R}^{2} \rightarrow \mathbf{R}^{2}$ em intervalos (piecewise linear maps) e máquinas de Turing universais, conforme encontrado em [Koiran et al., 1994] e [Blondel et al., 2000].

Proposição. Seja $M$ uma máquina de Turing e $C=\Sigma^{\omega} \times \Sigma^{\omega} \times Q$ seu espaço de configuração. Existe, então, um conjunto de funções lineares em intervalos $g_{M}: \mathbf{R}^{2} \rightarrow \mathbf{R}^{2}$ e uma função de codificação $v: C \rightarrow[0,1]^{2}$ de forma que $g_{M}(v(c))=v\left(c^{\prime}\right)$ para toda configuração $\left(c, c^{\prime}\right)$.

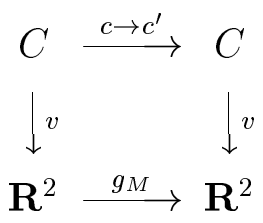

Demonstração [Blondel et al., 2000]. A demonstração é feita por construção direta das funções $v$ e $g_{M}$.

Considere uma configuração $\left(p_{1}, p_{2}, q\right)$ de máquina de Turing $M$, com $n$ símbolos e $m$ estados, sendo $p_{i}=a_{i}^{0} a_{i}^{1} a_{i}^{2} \ldots$ e $a_{j}^{i} \in \sum$. $p_{i}$ é codificado em um número real $x_{i}$ dado por:

$$
x_{i}=\sum_{j=0}^{\infty} \frac{2 a_{i}^{j}}{(2 n)^{j+1}}
$$

Tem-se, então, a função $v$ definida por:

$$
v\left(p_{1}, p_{2}, q\right)=\left(\frac{q}{m}+\frac{x_{1}}{m}, x_{2}\right)
$$

Para quaisquer $\alpha, \beta \in \sum$ e $q \in Q$, definem-se os seguintes subconjuntos disjuntos de $\mathbf{R}^{2}$ :

$$
B_{\alpha, \beta, q}=\left[\frac{q}{m}+\frac{2 \alpha}{2 m n}, \frac{q}{m}+\frac{2 \alpha+1}{2 m n}\right] \times\left[\frac{2 \beta}{2 n}, \frac{2 \beta+1}{2 n}\right]
$$


Pela definição, a imagem de $v$ de uma configuração da forma $\left(\alpha p_{1}^{\prime}, \beta p_{2}^{\prime}, q\right)$ é um ponto de $B_{\alpha, \beta, q}$.

Uma quíntupla de $M$ tem o efeito de trocar o símbolo $\alpha$, o símbolo corrente, por um símbolo $\alpha^{\prime}$, mantendo a fita em sua posição, ou movendo-a para direita ou para esquerda, e trocando o estado $q$ para $q^{\prime}$. Então, $g_{M}$ é definida para cada conjunto $B_{\alpha, \beta, q}$ por $g_{M}\left(q / m+x_{1} / m, x_{2}\right)=\left(q^{\prime} / m+x_{1}^{\prime} / m, x_{2}^{\prime}\right) \operatorname{com} x_{1}^{\prime}=a x_{1}+b$ e $x_{2}^{\prime}=c x_{2}+d$ com:

- se a fita de $M$ é movida para esquerda, $a=2 n, b=-2 \alpha, d=\left(2 \alpha^{\prime}\right) /(2 n)$

- se a fita de $M$ é movida para direita, $a=1 /(2 n), b=(2 \beta) /(2 n)+2\left(\alpha^{\prime}-\alpha\right) /(2 n)^{2}, c=2 n, d=-2 \beta$

- e, se a fita não é movida, $a=1, b=2\left(\alpha^{\prime}-\alpha\right) /(2 n), c=1, d=0$

E, desse modo, tem-se definidas $g_{M}(v(c))=v\left(c^{\prime}\right)$ para todo par de configurações $\left(c, c^{\prime}\right)$ ( fim da demonstração).

Observe que a construção acima produz um conjunto de funções lineares $g_{M}$ para qual o conjunto $S=\left(\bigcup B_{\alpha, \beta, q}\right) \cap[01]^{2}, \alpha, \beta \in \sum$ e $q \in Q$, é um conjunto invariante de $g_{M}$, isto é, $g_{M}\left(x_{1}, x_{2}\right) \in S$ para todo $\left(x_{1}, x_{2}\right) \in S$ e, portanto, sucessivas aplicações de $g_{M}$ recaem sobre $S^{6}$. Tem-se, portanto, um mapa definido $\left(x_{1}(t+1), x_{2}(t+1)\right)=g_{M}\left(x_{1}(t), x_{2}(t)\right)$ 7. O conjunto $S$ é um conjunto que se assemelha aos primeiros passos da construção do conjunto de Cantor e divide o intervalo $\left[\begin{array}{ll}0 & 1\end{array}\right]^{2}$ em $n^{2} \times m$ sub-intervalos disjuntos correspondentes aos $n$ símbolos e $m$ estados da máquina $M^{8}$. Por último, observe que todas as configurações de uma mesma quíntupla de uma máquina de Turing, mapeadas por $v$, são levadas ao mesmo subconjunto $B_{\alpha, \beta, q}$. O espaço invariante $S$ construído para uma máquina de 3 símbolos e 3 estados é apresentado na figura (5.4). Aplicamos, a seguir, essa abordagem para solução do problema da identificação da paridade de bits. Partimos da máquina de Turing definida em (5.1) empregando um estado e um símbolo adicionais, os quais embora não sejam requeridos, expõem melhor a funcionalidade desse modelo.

\footnotetext{
${ }^{6}$ Precisamente: um conjunto invariante $S$ de um mapa $g$ é um subconjunto $S \subset \mathbf{R}^{n}$ tal que $g(x) \in S$ para todo $t \in \mathbf{R}$ [Guckenheimer \& Holmes, 1983].

${ }^{7} \mathrm{O}$ conjunto $S$ garante que o domínio e contra-domínio de $g_{M}$ sejam iguais, permitindo sucessivas aplicações de $g_{M}$ de acordo com nossa definição de mapas (seção 2.1).

${ }^{8}$ Mas $S$ não é um conjunto de Cantor o qual, por definição, não contêm nenhum intervalo [Holmgren, 1996].
} 


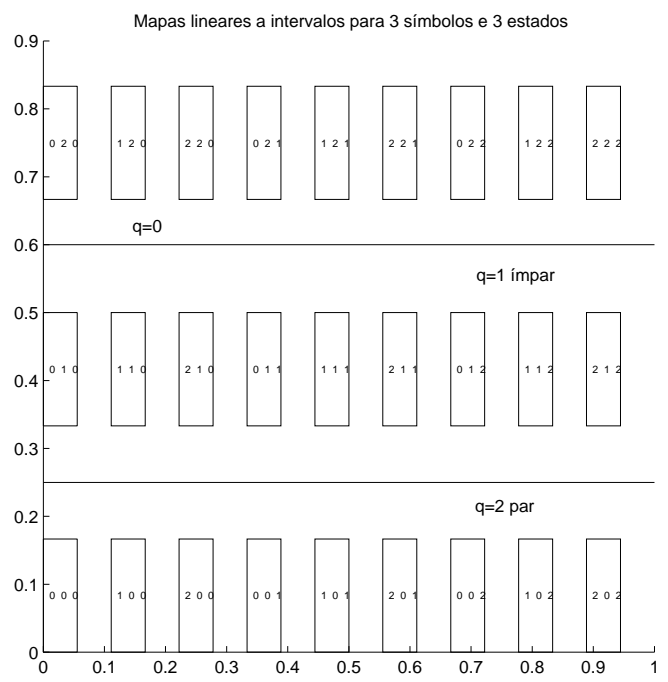

Figura 5.4: Espaço invariante $S$ gerado para emulação de uma máquina de Turing com 3 símbolos e 3 estados.

\section{Um exemplo}

Verificador de paridade. A construção do verificador de paridade de bits através de mapas lineares em intervalos em $[0,1]^{2}$ poderia fazer uso, como nos exemplos anteriores, de apenas dois símbolos 0 e 1 e dois estados, também representados por 0 e 1, para indicação da paridade ou não dos bits de uma seqüência. Entretanto, o uso de dois símbolos e dois estados resultaria em um espaço invariante $S$ reduzido de apenas 4 sub-intervalos em $\left[\begin{array}{ll}0 & 1\end{array}\right]^{2}$ e dificultaria apresentar claramente a funcionalidade do modelo. Adotamos, portanto, o modelo da figura (5.4) de 3 símbolos e 3 estados, adicionando o símbolo 2 para indicar o final da fita, sendo os estados 0 para condição inicial, 1 para o valor ímpar de bits e 2 para o valor par. Os resultados para as seqüência 1011 e 1010 são mostrados na figura (5.5). As flechas indicam o mapeamento das funções de $g_{M}$.

Tratou-se, até aqui, da capacidade de mapas, na verdade, um conjunto de mapas lineares em intervalos, emularem uma máquina de Turing arbitrária. A capacidade de computação universal desses mapas pode ser concluída da observação de que o modelo anterior implementa máquinas de Turing com quantidades de símbolos e estados arbitrários e, portanto, permite implementar máquinas de Turing universais como as propostas por Minsky (MTU(7,4), 7 estados e 4 símbolos) e Rogozhin $(\operatorname{MTU}(24,2)$ e MTU(10,3)) como considerado na seção (5.2). Para, por exemplo, a máquina de Minsky, MTU(7,4), a construção de mapas lineares em intervalos produz um conjunto de 112 sub-intervalos $\left[\begin{array}{ll}0 & 1\end{array}\right]^{2}$, $n^{2} \times m$, enquanto o modelo de Rogozhin, $\operatorname{MTU}(24,2)$, requer apenas 48 sub-intervalos. 

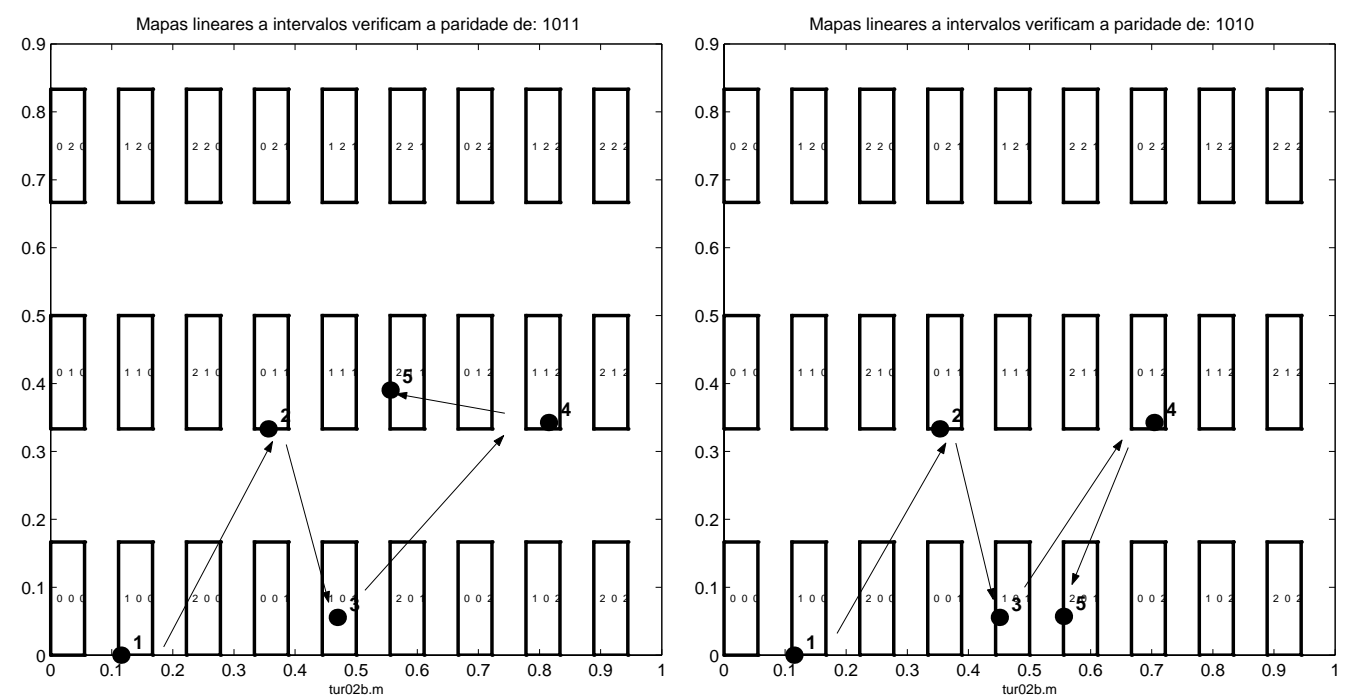

Figura 5.5: Emulação de uma máquina de Turing através de mapas lineares em intervalos para verificação da paridade de bits das seqüências 1011 e 1010.

\section{Em resumo: mapas exibem computação universal}

Em resumo, demonstra-se que é possível obter computação universal por um mapa $g_{M}$ : $\left[\begin{array}{ll}0 & 1\end{array}\right]^{2} \rightarrow\left[\begin{array}{ll}0 & 1\end{array}\right]^{2}$ definido como um conjunto de 48 transformações (considera-se a máquina de Rogozhin, $\operatorname{MTU}(24,2))$ da forma:

$$
g_{M_{k}}: B_{k} \rightarrow[01]^{2}, \quad g_{M_{k}}(x)=A_{k}+b_{k}
$$

sendo $x \in S \subset\left[\begin{array}{ll}0 & 1\end{array}\right]^{2}$ e $A_{k}, b_{k}$ constituídos dos coeficientes $a, b, c, d$ definidos na demonstração.

[Orponen \& Matamala, 1996] também demonstraram a capacidade de computação universal por mapas acoplando-os em uma rede do tipo CML, mas com conexão unidirecional (ver seção (2.7)) como na figura (5.6), em que cada elemento é um dos mapas $g_{M_{k}}$ de (5.10). Eles, entretanto, utilizaram a máquina de Minsky, sendo necessários 112 elementos acoplados. Parece-nos, nesse caso, que o uso da máquina Rogozhin, $\operatorname{MTU}(24,2)$, seria mais adequada.

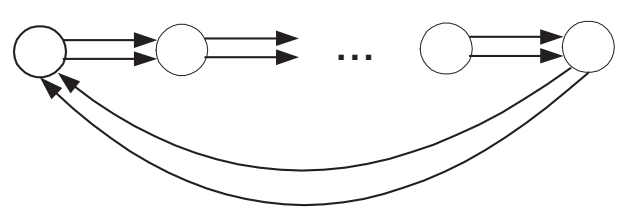

Figura 5.6: CML unidirecional. Cada elemento representa um mapa linear em $\mathbf{R}^{2}$. 


\subsection{Máquinas de Turing e o mapa do padeiro}

A abordagem do uso de mapas lineares em intervalos apresenta, dentre outros, o inconveniente óbvio do número de elementos requeridos e parece não trazer quaisquer vantagens sobre uma máquina de Turing, uma vez que os mapas são gerados a partir de uma máquina de Turing prévia. Não obstante, trata-se de um resultado importante. Particularmente, a idéia de um conjunto invariante sobre as funções que emulam uma máquina de Turing parece estar na base diversos outros modelos de computação baseados em sistemas dinâmicos.

No próximo modelo que consideramos, mapas do padeiro (baker's map) (ver exemplo (2.4)) são adaptados para fornecer computação universal. Esse modelo é descrito em [Sato et al., 2000] e basicamente generaliza o trabalho anterior, dentre outros, de [Moore, 1990 ${ }^{9}$, introduzindo o conceito de sistemas de mapas com funções de troca (em [Sato et al., 2000]: switching map systems). Consideramos esse modelo particularmente interessante e optamos por seu uso em nossa aplicação final de tratamento simbólico para imagens. A seguir, introduzem-se os conceitos básicos envolvidos nesse modelo e sua aplicação no problema da verificação de paridade de bits.

\section{Sistemas de mapas com funções de troca}

Definição [Sato et al., 2000] Sejam $f_{1}, f_{2}, \ldots, f_{m}$ mapas em $X \subset \mathbf{R}^{n}$. Define-se $F$ um sistema de mapas com função de troca como um sistema $F\left(f_{1}, f_{2}, \ldots, f_{m}\right)$, tal que $F$ : $S \times X \rightarrow S \times X:(n, x) \mapsto\left(g_{n}(x), f_{m}(x)\right)$, sendo $S=0, \ldots, N-1$ um conjunto de $N$ estados internos, $n \in S$ e $N$ funções de troca ${ }^{10} g_{1}, g_{2}, \ldots, g_{N}, g: X \rightarrow S$.

Observe que, na prática, esse sistema de mapas com funções de troca permite, a partir de uma configuração inicial $\left(n_{0}, x_{0}\right) \in S \times X$, alterar, a cada passo a dinâmica do sistema, como segue:

\footnotetext{
${ }^{9}$ Basicamente Moore [Moore, 1990] introduz o conceito de generalized shift map, um shift map como é encontrado no estudo de dinâmica simbólica (ver [Devaney, 1989] [Holmgren, 1996]) mas que alterna deslocamentos para direita, ou para esquerda, de acordo com uma função da seqüência de símbolos na qual é aplicado o mapa aos inteiros 1 (direita) ou -1 (esquerda).

${ }^{10} \mathrm{Em}$ [Sato et al., 2000]: branching functions.
} 


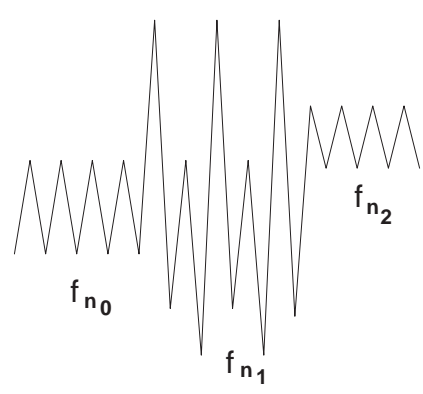

Figura 5.7: Representação da troca da dinâmica em sistemas de mapas com funções de troca.

$$
\begin{aligned}
\left(n_{0}, x_{0}\right) & \mapsto\left(g_{n_{0}}\left(x_{0}\right), f_{n_{0}}\left(x_{0}\right)\right)=\left(n_{1}, x_{1}\right) \\
& \mapsto\left(g_{n_{1}}\left(x_{1}\right), f_{n_{1}}\left(x_{1}\right)\right)=\left(n_{2}, x_{2}\right) \\
\cdots & \\
& \mapsto\left(g_{n_{t}}\left(x_{t}\right), f_{n_{t}}\left(x_{t}\right)\right)=\left(n_{t+1}, x_{t+1}\right)
\end{aligned}
$$

\section{Mapa do padeiro}

Demonstra-se [Sato et al., 2000] que sistemas de mapas com função de troca considerandose $F\left(B_{0}, B_{1}^{-1}, I\right)$, sendo $I$ a identidade e $B_{0}, B_{1}$ mapas do padeiro como:

$$
\begin{aligned}
& B_{0}(x, y)=\left\{\begin{array}{cc}
(2 x, y / 2) & \text { se } 0 \leq x<1 / 2 \\
\left(2 x-1, \frac{y+1}{2}\right) & \text { se } 1 / 2 \leq x_{t}<1
\end{array}\right. \\
& B_{1}(x, y)=\left\{\begin{array}{cc}
\left(2 x, \frac{y+1}{2}\right) & \text { se } 0 \leq x<1 / 2 \\
(2 x-1, y / 2) & \text { se } 1 / 2 \leq x_{t}<1
\end{array}\right.
\end{aligned}
$$

exibem computação universal.

Embora a prova em [Sato et al., 2000] seja elaborada, observamos que essas funções correspondem exatamente a operações de shift e de troca de valores sobre uma seqüência de valores binários (a multiplicação por 2 leva seqüências binárias para esquerda, a multiplicação por 1/2 leva seqüências binárias para direita, e somas e substrações de uma unidade alternam o valor de um símbolo da seqüência). 
As funções $B_{0}, B_{1}$ e $I$ constituem nas operações elementares do sistema e, em conjunto com as funções de troca $g_{i}$, buscam tornar o sistema "programável". Em [Sato et al., 2000], esse modelo é aplicado para solução do problema de verificação de paridade de bits e reproduzimos aqui de modo mais detalhado o experimento realizado. Isso foi importante para nos familiarizar com esse tipo de "programação", que é aplicada em novos contextos na próxima seção.

Novamente, consideramos a máquina de Turing:

\begin{tabular}{ccccc}
$q_{i}$ & $s_{j}$ & $s_{i j}$ & $d_{i j}$ & $q_{i j}$ \\
\hline 0 & 0 & 0 & -1 & 0 \\
0 & 1 & 1 & -1 & 1 \\
1 & 0 & 0 & -1 & 1 \\
1 & 1 & 1 & -1 & 0
\end{tabular}

Para essa máquina, associamos o seguinte sistema de mapas com funções de troca:

$$
\begin{aligned}
(0,(x, y)) & \mapsto\left(0, B_{0}(x, y)\right) \\
(0,(x, y)) & \mapsto\left(1, B_{1}(x, y)\right) \\
(1,(x, y)) & \mapsto\left(1, B_{1}(x, y)\right) \\
(1,(x, y)) & \mapsto \quad\left(0, B_{0}(x, y)\right)
\end{aligned}
$$

sendo $B_{0}$ e $B_{1}$ baker's maps como definidos em (5.12) e (5.13), e $g_{0}$ a função:

$$
g_{0}(x, y)=g_{1}(x, y)= \begin{cases}0 & \text { se } y \leq 1 / 2 \\ 1 & \text { se } y>1 / 2\end{cases}
$$

Os resultados da simulação desse modelo são apresentados na figura 5.8.

\subsection{Modelo para computação não convencional}

No sistema de mapas com funções de troca (5.14) foram empregados mapas do padeiro como operações elementares do sistema. Com eles, emulamos uma máquina para verificação de paridade, sendo, ainda, possível emular qualquer máquina de Turing [Sato et al., 2000]. Pode-se, entretanto, adotar outros tipos de mapas como "operações elementares" do sistema, fornecendo um novo modelo de computação sobre números reais. [Sato et al., 2000] 

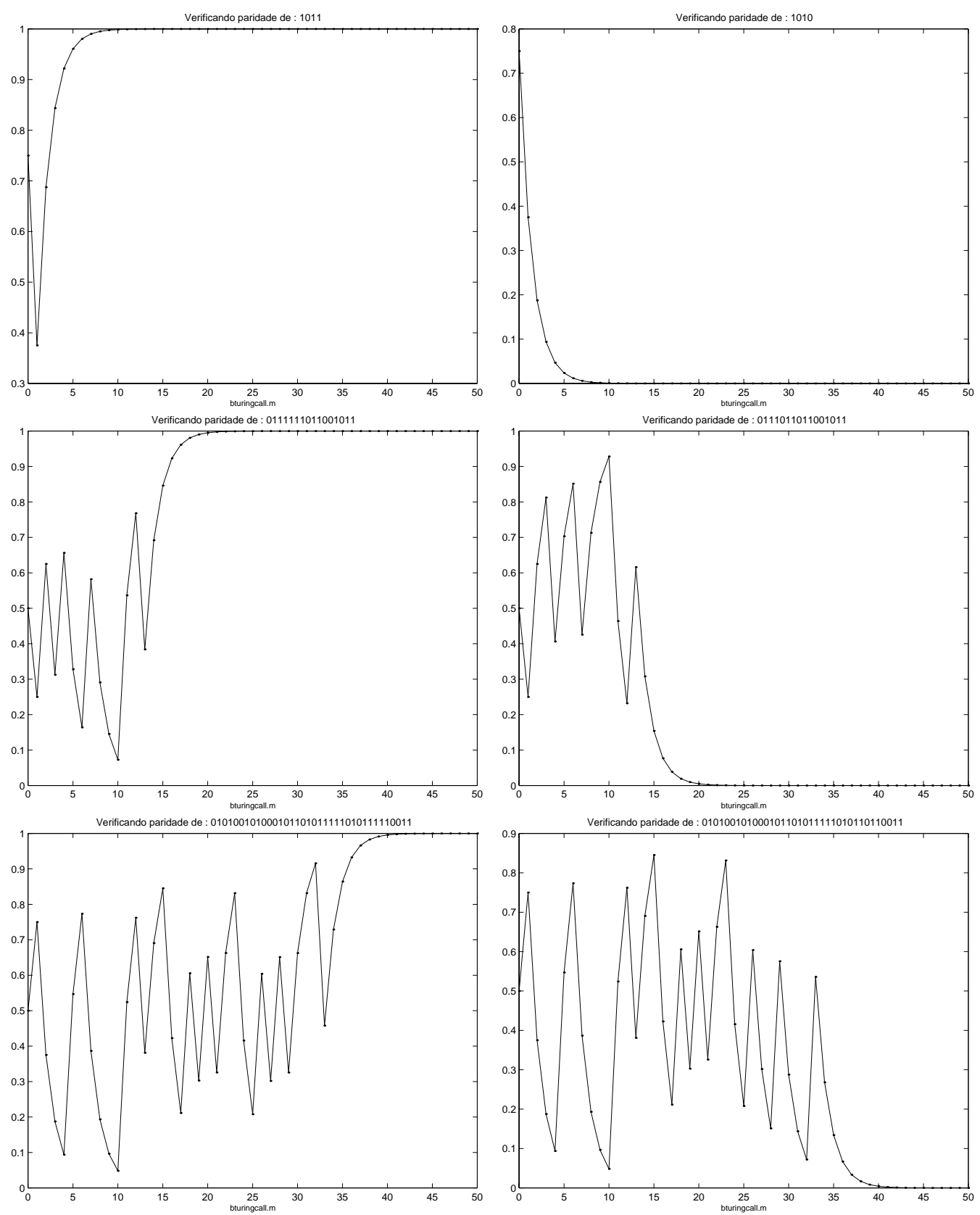

Figura 5.8: Mapas do padeiro em um sistema de funções de troca emulando uma máquina de Turing para verificação da paridade de bits. São exibidas seis seqüências diferentes de bits. À esquerda, encontram-se seqüências com quantidades ímpares de 1's; à direita seqüências com quantidades pares. Há a convergência para o valor correto, após algumas dezenas de iterações. 
sugere o uso de mapas não lineares, como o mapa de Hénon, como operações elementares desses sistemas, de modo a prover uma máquina de Turing e um modelo de computação "não lineares".

Novos modelos de computação, como esse, são de grande interesse e buscam superar alguns limites da computação tradicional [Blum et al., 1997], [Mertens, 2002]. A exemplo de outros modelos de computação não convencionais, como computação quântica e computação molecular ${ }^{11}$, o uso prático desses modelos ainda é objeto de investigação.

Nesta tese, adotamos transformações e mapas lineares no plano como operações elementares dos sistemas de mapas com funções de troca, e propomos uma aplicação desse modelo no processamento gráfico, como alternativa ao processamento simbólico de imagens realizado, por exemplo, empregando L-sistemas. O modelo que resulta é semelhante a sistemas de funções iteradas [Barnsley, 1988]. Uma revisão sobre funções iteradas encontra-se no anexo B. Antes de apresentar as aplicações do modelo, são introduzidos alguns conceitos de processamento simbólico de imagens.

\section{$5.7 \quad L$-sistemas}

L-sistemas (Lindenmayer systems) são um tipo particular de sistemas introduzidos por Lindenmayer (ver [Prusinkiewicz \& Hanan, 1989]) para representar graficamente o crescimento de sistemas biológicos. Mais recentemente, encontraram aplicação na computação gráfica, na geração de padrões fractais e na construção de imagens de plantas [Prusinkiewicz \& Hanan, 1989]; e na modelagem biológica em geral [Zamir, 2001]; sendo um dos principais sistemas empregados para geração de imagens através de uma linguagem formal de símbolos (uma gramática).

Um L-sistema é um sistema dinâmico simbólico que opera sobre um conjunto $\Sigma$ de símbolos (alfabeto) através de regras de produção e a partir de um conjunto inicial de símbolos (axioma, na linguagem dos L-sistemas).

Fibonacci. Considere o alfabeto $\Sigma=\{a, b\}$ e duas regras de produção dadas $p_{1}: a \mapsto b$ e $p_{2}: b \mapsto b a$, isto é, dada uma cadeia de símbolos, $p_{1}$ produz o símbolo $b$ para cada símbolo $a$ e $p_{2}$ produz a seqüência $b a$ para cada símbolo $b$. As seqüências produzidas $\omega_{i}$ a partir

\footnotetext{
${ }^{11}$ Para uma introdução a alguns modelos de computação não convencional, ver [Gramß et al., 1998].
} 


\begin{tabular}{c|c}
$\omega_{0}$ & $a$ \\
$\omega_{1}$ & $b$ \\
$\omega_{2}$ & $b a$ \\
$\omega_{3}$ & $b a b$ \\
$\omega_{3}$ & $b a b b a$ \\
$\omega_{3}$ & $b a b b a b a b$ \\
$\omega_{3}$ & $b a b b a b a b b a b b a$ \\
$\ldots$ & $\ldots$
\end{tabular}

Tabela 5.3: Sucessivas cadeiais de símbolos produzidas pelo L-sistema (5.16). O número de elementos produzidos corresponde a seqüência de Fibonacci 1, 1, 2, 3, 5, 8, 13, .. .

de uma seqüência inicial $\omega_{0}=\{a\}$ encontram-se na tabela 5.3. O número de símbolos da cadeia produzida a cada aplicação das regras de produção $p_{1}$ e $p_{2}$ é igual ao produzido pela seqüência de Fibonacci ${ }^{12}$.

$$
\begin{aligned}
& \Sigma=\{a, b\} \\
& \omega_{0}=a \\
& p_{1}: a \mapsto b \\
& p_{2}: \quad b \mapsto b a
\end{aligned}
$$

A interpretação geométrica dos símbolos de um L-sistema para a construção de objetos gráficos, como padrões fractais, é realizada com base nos mesmos conceitos usados para descrever um percurso da "tartaruga" na linguagem LOGO ${ }^{13}$. O estado da tartaruga é dado pela tripla $(x, y, \alpha)$, sendo $(x, y)$ as coordenadas cartesianas da posição da tartaruga e $\alpha$ o ângulo que indica a posição da "cabeça da tartaruga", direção na qual ela fará seu próximo movimento. Define-se, então, os seguintes símbolos e suas interpretações:

- F: a tartaruga avança uma distância $d$ na direção à sua frente.

- +: a cabeça gira no sentido horário de um ângulo de $\delta$.

- - : a cabeça gira no sentido anti-horário de um ângulo de $\delta$.

A distância $d$ e o ângulo $\delta$ são, em geral, fixos e os objetos gráficos são obtidos desenhando-se a trajetória da tartaruga.

\footnotetext{
${ }^{12}$ A seqüência de Fibonacci é uma seqüência de inteiros dada por $s_{0}=s_{1}=1$ e $s_{n}=s_{n-1}+s_{n}$ para $n \geq 2$.

${ }^{13}$ Linguagem desenvolvida por Seymour Papert, nos anos 80, para o aprendizado de crianças. Nela, o trajeto de uma "tartaruga" artificial é produzido através de um conjunto simples de seqüências de símbolos: ande para frente, vire à direita, vire à esquerda etc.
} 

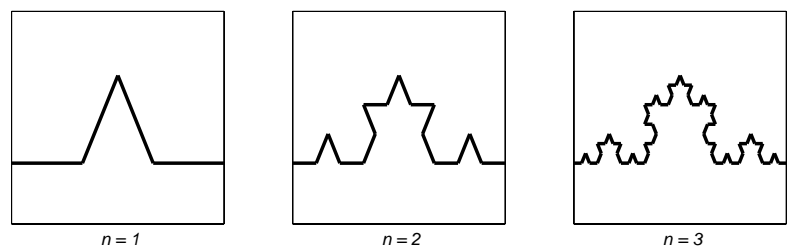

Figura 5.9: Os três primeiros estágios da construção da curva de Koch através do $L$ sistema (5.17).

Curva de Koch. A curva de Koch é uma estrutura fractal ${ }^{14}$ obtida como uma variação da construção do conjunto de Cantor [Peitgen et al., 1992]. Os primeiros estágios da construção da curva são apresentados na figura (5.9). A curva de Koch pode ser produzida, empregando-se a interpretação geométrica dos símbolos $\mathbf{F},+$ e -, pelo seguinte L-sistema:

$$
\begin{aligned}
\Sigma & =\{F,+,-\} \\
\omega_{0} & =F \\
p & : F \mapsto F+F--F+F
\end{aligned}
$$

sendo $d \in \mathbf{R}$ um valor de comprimento arbitrário e $\delta=\pi / 3$.

Os conceitos apresentados definem L-sistema como sendo determinista e uma linguagem livre de contexto ${ }^{15}$. Esses sistemas ainda podem ser estendidos para construção de linguagens mais complexas e que são capazes de efetuar computação. Entre essas extensões, encontram-se L-sistemas dependentes de contexto, estocáticos e bracketed $L$ systems. Uma revisão é encontrada em [Prusinkiewicz \& Hanan, 1989].

\subsection{Processamento simbólico e mapas}

Nosso modelo adota sistemas de mapas com funções de troca, empregando mapas de um modo geral, como operações elementares para a realização de processamento gráfico, como a geração de padrões fractais, texturas e estruturas de plantas. A seguir, utilizamos mapas da forma $f:\left(x_{1}, x_{2}, \alpha\right) \rightarrow\left(x_{1}^{\prime}, x_{2}^{\prime}, \alpha^{\prime}\right)$, sendo $\left(x_{1}, x_{2}\right),\left(x_{1}^{\prime}, x_{2}^{\prime}\right)$ as coordenadas cartesianas e $\alpha, \alpha^{\prime}$ ângulos com a mesma interpretação gráfica empregada nos $L$-sistemas. Empregamos mapas lineares no plano. Essas aplicações são, segundo nosso conhecimento, novas.

\footnotetext{
${ }^{14}$ Basta, aqui, considerar-se estruturas que exibem auto-similaridade.

${ }^{15}$ Basicamente: as regras são fixas e idênticas para quaisquer valores da cadeia.
} 


\section{Mapas $f:\left(x_{1}, x_{2}, \alpha\right) \rightarrow\left(x_{1}^{\prime}, x_{2}^{\prime}, \alpha^{\prime}\right)$}

Considere o sistema de mapas com função de troca:

$$
F:(n, x) \mapsto\left(g_{n}(x), f_{m}(x)\right)
$$

com $x=\left(x_{1}, x_{2}, \alpha\right)$, sendo $\left(x_{1}, x_{2}\right)$ coordenadas cartesianas em $\mathbf{R}^{2}, \alpha \in \mathbf{R}$ um ângulo e as operações elementares dadas por:

$$
f_{i}(x)=f_{i}\left(x_{1}, x_{2}, \alpha\right)=\left(x_{1}+d \cos \alpha, x_{2}+d \sin \alpha, \alpha+\delta\right)
$$

sendo $d, \delta \in \mathbf{R}$ parâmetros da transformação $f_{i}$, e as funções de troca $g_{n}$ escolhidas de forma a promoverem a troca desejada das transformações.

O sistema (5.18) pode emular qualquer L-sistema: as equações (5.19) emulam as operações $\mathbf{F},+$ e - , do L-sistema com os parâmetros $d$ e $\delta$; e as funções de troca $g_{n}$ dão o passo e a troca das operações.

Um simples exemplo: a curva de Koch. A curva de Koch foi construída anteriormente pela regra de produção $p: F \mapsto F+F--F+F$ de um $L$-sistema, é agora obtida por um sistema de mapas com funções de troca como segue:

$$
\begin{aligned}
F_{k}:(n, x) & \mapsto\left(g_{n}(x), f_{m}(x)\right) \\
f_{1}(x) & =f_{1}\left(x_{1}, x_{2}, \alpha\right)=\left(x_{1}+\frac{d \cos \alpha}{3 k}, x_{2}+\frac{d \sin \alpha}{3 k}, \alpha+0 \pi / 6\right) \\
f_{2}(x) & =f_{2}\left(x_{1}, x_{2}, \alpha\right)=\left(x_{1}+\frac{d \cos \alpha}{3 k}, x_{2}+\frac{d \sin \alpha}{3 k}, \alpha+2 \pi / 6\right) \\
f_{3}(x) & =f_{3}\left(x_{1}, x_{2}, \alpha\right)=\left(x_{1}+\frac{d \cos \alpha}{3 k}, x_{2}+\frac{d \sin \alpha}{3 k}, \alpha-4 \pi / 6\right) \\
f_{4}(x) & =f_{4}\left(x_{1}, x_{2}, \alpha\right)=\left(x_{1}+\frac{d \cos \alpha}{3 k}, x_{2}+\frac{d \sin \alpha}{3 k}, \alpha+2 \pi / 6\right) \\
g_{n}(x) & =n+1
\end{aligned}
$$

A exemplo de sistemas de funções iteradas (ver anexo B), $F_{k}$ é aplicada a todos os pontos do contra-domínio $A$ de $F_{k-1}$, sendo, ainda, $(0,0,0)$ a condição inicial. A construção de objetos gráficos é feita pela representação dos pontos das coordenadas $\left(x_{1}, x_{2}\right)$ no plano, com linhas ligando ou não esses pontos. A figura (5.10) mostra as 

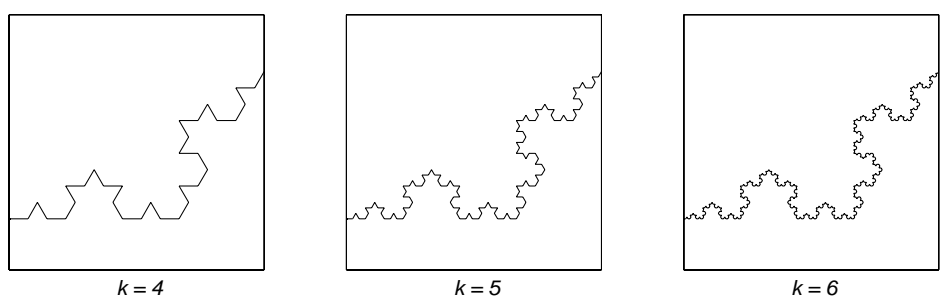

Figura 5.10: Curva de Koch produzida pelo sistema (5.20).

curvas de Koch obtidas.

\section{Ilha de Koch e mutações}

A ilha de Koch é uma forma geométrica apresentada na figura (5.11) que pode ser obtida por um L-sistema da forma:

$$
\begin{aligned}
\Sigma & =\{F,+,-\} \\
\omega_{0} & =F+F+F+F \\
p & : F \mapsto F+F-F F+F+F-F
\end{aligned}
$$

sendo $d \in \mathbf{R}$ um valor de comprimento arbitrário e $\delta=\pi / 2$.

A mesma forma é produzida pelo seguinte sistema de mapas com funções de troca:

$$
\begin{aligned}
F_{k}:(n, x) & \mapsto\left(g_{n}(x), f_{m}(x)\right) \\
f_{1}(x) & =f_{1}\left(x_{1}, x_{2}, \alpha\right)=\left(x_{1}+\frac{d \cos \alpha}{4 k}, x_{2}+\frac{d \sin \alpha}{4 k}, \alpha+0\right) \\
f_{2}(x) & =f_{2}\left(x_{1}, x_{2}, \alpha\right)=\left(x_{1}+\frac{d \cos \alpha}{4 k}, x_{2}+\frac{d \sin \alpha}{4 k}, \alpha-\pi / 2\right) \\
f_{3}(x) & =f_{3}\left(x_{1}, x_{2}, \alpha\right)=\left(x_{1}+\frac{d \cos \alpha}{4 k}, x_{2}+\frac{d \sin \alpha}{4 k}, \alpha+\pi / 2\right) \\
f_{4}(x) & =f_{4}\left(x_{1}, x_{2}, \alpha\right)=\left(x_{1}+\frac{d \cos \alpha}{4 k}, x_{2}+\frac{d \sin \alpha}{4 k}, \alpha+\pi / 2\right) \\
f_{5}(x) & =f_{5}\left(x_{1}, x_{2}, \alpha\right)=\left(x_{1}+\frac{d \cos \alpha}{4 k}, x_{2}+\frac{d \sin \alpha}{4 k}, \alpha+0\right) \\
f_{6}(x) & =f_{6}\left(x_{1}, x_{2}, \alpha\right)=\left(x_{1}+\frac{d \cos \alpha}{4 k}, x_{2}+\frac{d \sin \alpha}{4 k}, \alpha-\pi / 2\right) \\
f_{7}(x) & =f_{7}\left(x_{1}, x_{2}, \alpha\right)=\left(x_{1}+\frac{d \cos \alpha}{4 k}, x_{2}+\frac{d \sin \alpha}{4 k}, \alpha-\pi / 2\right)
\end{aligned}
$$




$$
\begin{aligned}
& f_{8}(x)=f_{8}\left(x_{1}, x_{2}, \alpha\right)=\left(x_{1}+\frac{d \cos \alpha}{4 k}, x_{2}+\frac{d \sin \alpha}{4 k}, \alpha+\pi / 2\right) \\
& g_{n}(x)=n+1
\end{aligned}
$$

sendo $F_{k}$ aplicada a todos os pontos do contra-domínio $A$ de $F_{k-1}$, sendo, e $(0,0,0)$ a condição inicial. A figura (5.11) apresenta a ilhas de Koch produzidas.

Os sistemas de mapas com funções de troca, como empregados em (5.20) e (5.22), são mais flexíveis do que L-sistemas deterministas e livres de contexto. A representação direta das transformações permite uma maior diversidade de transformações, utilizando-se diferentes parâmetros, e, portanto, uma maior diversidade de formas pode ser produzida. As figuras (5.12) e (5.13) apresentam "mutações" da forma original da ilha de Koch e foram produzidas pelos sistemas de mapas com funções de troca (5.23) e (5.24) que seguem.

$$
\begin{aligned}
F_{k}:(n, x) & \mapsto\left(g_{n}(x), f_{m}(x)\right) \\
f_{1}(x) & =f_{1}\left(x_{1}, x_{2}, \alpha\right)=\left(x_{1}+\frac{d \cos \alpha}{4 k}, x_{2}+\frac{d \sin \alpha}{4 k}, \alpha+0\right) \\
f_{2}(x) & =f_{2}\left(x_{1}, x_{2}, \alpha\right)=\left(x_{1}+\frac{d \cos \alpha}{4 k}, x_{2}+\frac{d \sin \alpha}{4 k}, \alpha-\pi / 3\right) \\
f_{3}(x) & =f_{3}\left(x_{1}, x_{2}, \alpha\right)=\left(x_{1}+\frac{d \cos \alpha}{4 k}, x_{2}+\frac{d \sin \alpha}{4 k}, \alpha+\pi / 2\right) \\
f_{4}(x) & =f_{4}\left(x_{1}, x_{2}, \alpha\right)=\left(x_{1}+\frac{d \cos \alpha}{4 k}, x_{2}+\frac{d \sin \alpha}{4 k}, \alpha+\pi / 2\right) \\
f_{5}(x) & =f_{5}\left(x_{1}, x_{2}, \alpha\right)=\left(x_{1}+\frac{d \cos \alpha}{4 k}, x_{2}+\frac{d \sin \alpha}{4 k}, \alpha+0\right) \\
f_{6}(x) & =f_{6}\left(x_{1}, x_{2}, \alpha\right)=\left(x_{1}+\frac{d \cos \alpha}{4 k}, x_{2}+\frac{d \sin \alpha}{4 k}, \alpha-\pi / 3\right) \\
f_{7}(x) & =f_{7}\left(x_{1}, x_{2}, \alpha\right)=\left(x_{1}+\frac{d \cos \alpha}{4 k}, x_{2}+\frac{d \sin \alpha}{4 k}, \alpha-\pi / 2\right) \\
f_{8}(x) & =f_{8}\left(x_{1}, x_{2}, \alpha\right)=\left(x_{1}+\frac{d \cos \alpha}{4 k}, x_{2}+\frac{d \sin \alpha}{4 k}, \alpha+\pi / 2\right) \\
g_{n}(x) & =n+1
\end{aligned}
$$

$$
\begin{aligned}
F_{k}:(n, x) & \mapsto\left(g_{n}(x), f_{m}(x)\right) \\
f_{1}(x) & =f_{1}\left(x_{1}, x_{2}, \alpha\right)=\left(x_{1}+\frac{d \cos \alpha}{4 k}, x_{2}+\frac{d \sin \alpha}{4 k}, \alpha+0\right) \\
f_{2}(x) & =f_{2}\left(x_{1}, x_{2}, \alpha\right)=\left(x_{1}+\frac{d \cos \alpha}{4 k}, x_{2}+\frac{d \sin \alpha}{4 k}, \alpha-\pi / 2\right) \\
f_{3}(x) & =f_{3}\left(x_{1}, x_{2}, \alpha\right)=\left(x_{1}+\frac{d \cos \alpha}{4 k}, x_{2}+\frac{d \sin \alpha}{4 k}, \alpha+\pi / 2\right)
\end{aligned}
$$



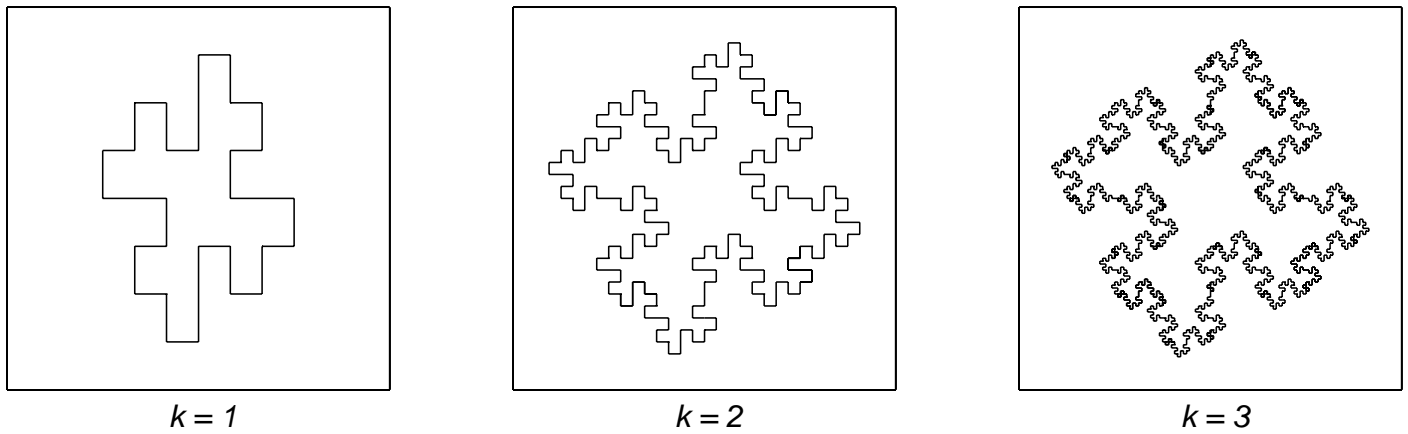

Figura 5.11: Ilha de Koch original produzida pelo sistema (5.22).

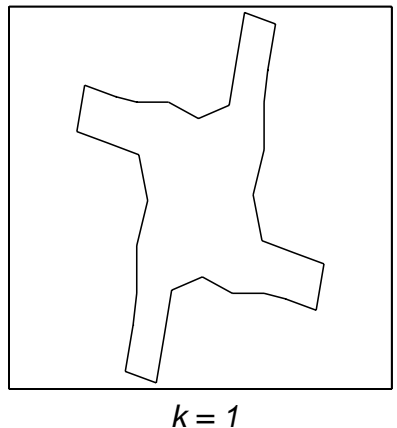

$k=1$
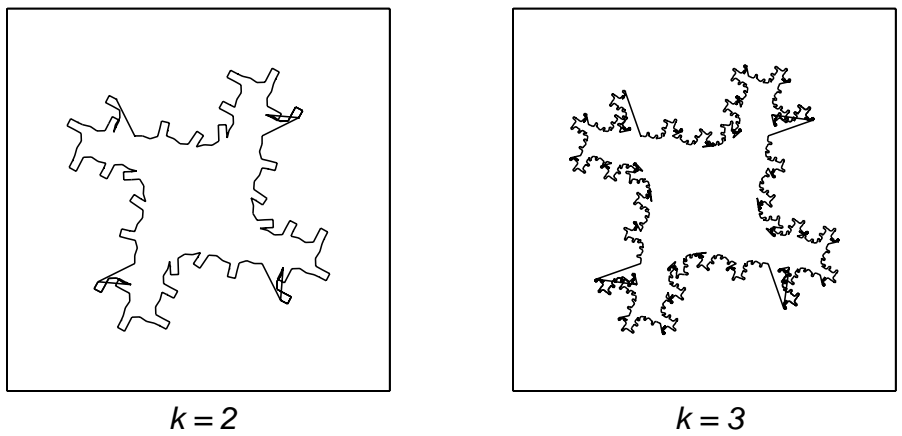

Figura 5.12: "Mutações" da ilha de Koch produzidas pelo sistema (5.23).

$$
\begin{aligned}
& f_{4}(x)=f_{4}\left(x_{1}, x_{2}, \alpha\right)=\left(x_{1}+\frac{d \cos \alpha}{4 k}, x_{2}+\frac{d \sin \alpha}{4 k}, \alpha+\pi / 2\right) \\
& f_{5}(x)=f_{5}\left(x_{1}, x_{2}, \alpha\right)=\left(x_{1}+\frac{d \cos \alpha}{4 k}, x_{2}+\frac{d \sin \alpha}{4 k}, \alpha+0\right) \\
& f_{6}(x)=f_{6}\left(x_{1}, x_{2}, \alpha\right)=\left(x_{1}+\frac{d \cos \alpha}{4 k}, x_{2}+\frac{d \sin \alpha}{4 k}, \alpha-\pi / 2\right) \\
& f_{7}(x)=f_{7}\left(x_{1}, x_{2}, \alpha\right)=\left(x_{1}+\frac{d \cos \alpha}{4 k}, x_{2}+\frac{d \sin \alpha}{4 k}, \alpha-\pi / 2\right) \\
& f_{8}(x)=f_{8}\left(x_{1}, x_{2}, \alpha\right)=\left(x_{1}+\frac{d \cos \alpha}{4 k}, x_{2}+\frac{d \sin \alpha}{4 k}, \alpha+\pi / 2\right) \\
& g_{n}(x)=n+1
\end{aligned}
$$
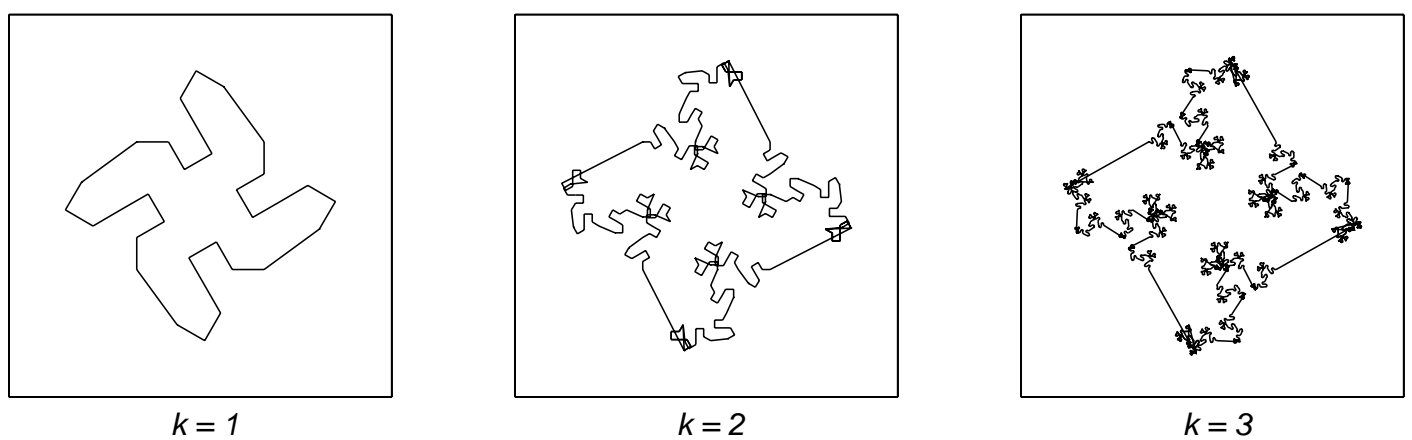

Figura 5.13: "Mutações" da ilha de Koch produzidas pelo sistema (5.24). 

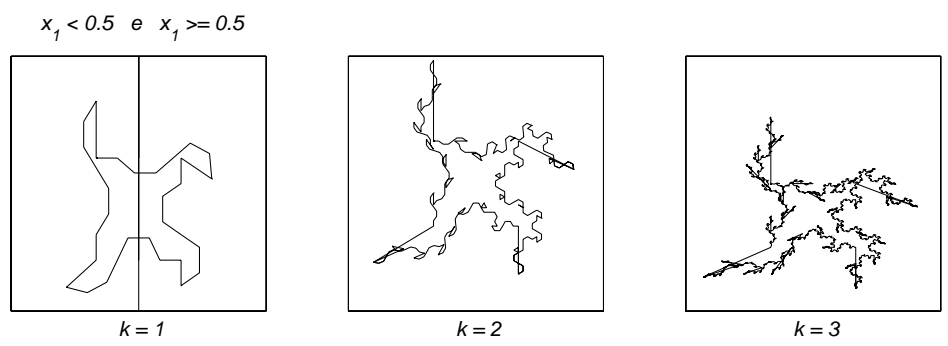

Figura 5.14: Forma obtida pelas transformações (5.25) sensíveis ao contexto .

Os sistemas anteriores apresentam funções de troca bastante simples $g_{n}(x)=n+1$, que não se alteram para diferentes valores de $x$. O uso de funções de troca que levam a diferentes operações elementares, segundo o valor $x$, tornam a programação com esses sistemas sensíveis ao contexto. Isso permite criar outros padrões de formas, além dos produzidos pela emulação dos L-sistemas aqui empregados ${ }^{16}$. Esses sistemas fornecem, assim, uma fonte de variedade de formas. As figuras de (5.14) foram construídas à partir das equações $(5.25)$ em que $g_{n}(x)$ leva a diferentes transformações $f_{i}$, segundo o valor de $x$.

$$
\begin{aligned}
& F_{k}:(n, x) \quad \mapsto \quad\left(g_{n}(x), f_{m}(x)\right) \\
& f_{1, \delta}(x)=f_{1}\left(x_{1}, x_{2}, \alpha\right)=\left(x_{1}+\frac{d \cos \alpha}{4 k}, x_{2}+\frac{d \sin \alpha}{4 k}, \alpha+0\right) \\
& f_{2, \delta}(x)=f_{2}\left(x_{1}, x_{2}, \alpha\right)=\left(x_{1}+\frac{d \cos \alpha}{4 k}, x_{2}+\frac{d \sin \alpha}{4 k}, \alpha-\delta\right) \\
& f_{3, \delta}(x)=f_{3}\left(x_{1}, x_{2}, \alpha\right)=\left(x_{1}+\frac{d \cos \alpha}{4 k}, x_{2}+\frac{d \sin \alpha}{4 k}, \alpha+\delta\right) \\
& f_{4, \delta}(x)=f_{4}\left(x_{1}, x_{2}, \alpha\right)=\left(x_{1}+\frac{d \cos \alpha}{4 k}, x_{2}+\frac{d \sin \alpha}{4 k}, \alpha+\delta\right) \\
& f_{5, \delta}(x)=f_{5}\left(x_{1}, x_{2}, \alpha\right)=\left(x_{1}+\frac{d \cos \alpha}{4 k}, x_{2}+\frac{d \sin \alpha}{4 k}, \alpha+0\right) \\
& f_{6, \delta}(x)=f_{6}\left(x_{1}, x_{2}, \alpha\right)=\left(x_{1}+\frac{d \cos \alpha}{4 k}, x_{2}+\frac{d \sin \alpha}{4 k}, \alpha-\delta\right) \\
& f_{7, \delta}(x)=f_{7}\left(x_{1}, x_{2}, \alpha\right)=\left(x_{1}+\frac{d \cos \alpha}{4 k}, x_{2}+\frac{d \sin \alpha}{4 k}, \alpha-\delta\right) \\
& f_{8, \delta}(x)=f_{8}\left(x_{1}, x_{2}, \alpha\right)=\left(x_{1}+\frac{d \cos \alpha}{4 k}, x_{2}+\frac{d \sin \alpha}{4 k}, \alpha+\delta\right) \\
& g_{n}(x)=g_{n}\left(x_{1}, x_{2}\right)= \begin{cases}n+1, \delta=\pi / 5 & \text { se } x_{1}<1 / 2 \\
n+1, \delta=\pi / 3 & \text { se } x_{1} \geq 1 / 2\end{cases}
\end{aligned}
$$

Uma das principais aplicações de L-sistemas é a reprodução de padrões botânicos [Prusinkiewicz \& Hanan, 1989]. Elaboramos, a seguir, um exemplo que busca produzir

\footnotetext{
${ }^{16}$ Cabe ressaltar que outros símbolos podem ser adicionados a L-sistemas tornando-os, também, linguagens sensíveis ao contexto.
} 

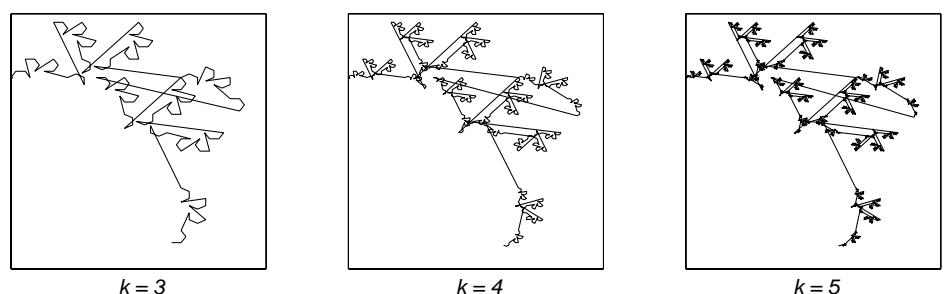

Figura 5.15: Padrão "botânico"produzido pelo sistema de equações (5.26).

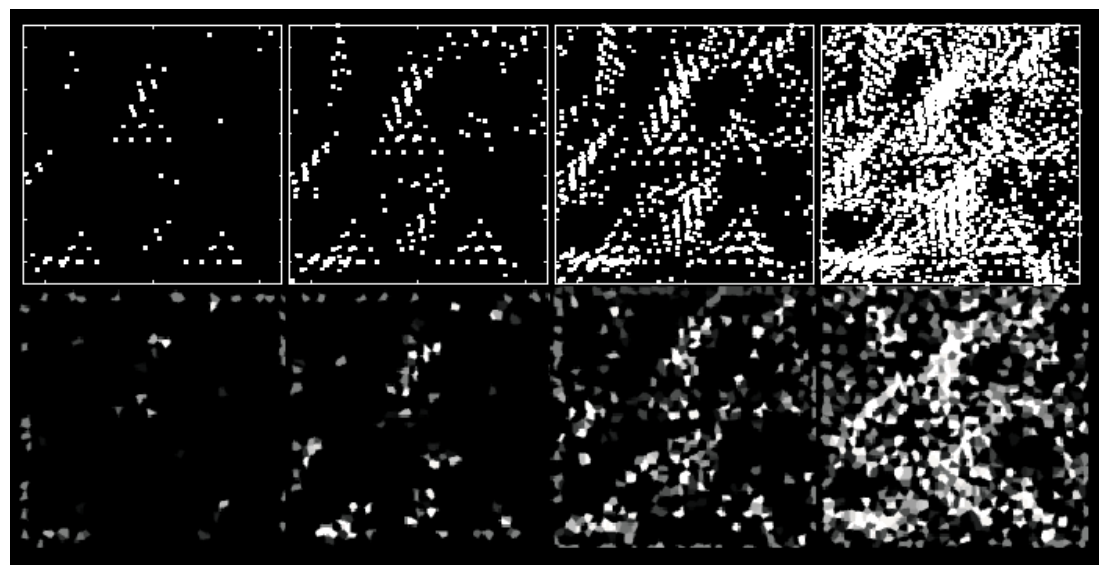

Figura 5.16: Representação de um processo de difusão não uniforme em uma superfície.

uma forma "botânica" empregando nosso modelo com o seguinte conjunto de equações:

$$
\begin{aligned}
F_{k}:(n, x) & \mapsto\left(g_{n}(x), f_{m}(x)\right) \\
f_{1}(x) & =f_{1}\left(x_{1}, x_{2}, \alpha\right)=\left(x_{1}+\frac{d \cos \alpha}{3 k}, x_{2}+\frac{d \sin \alpha}{3 k}, \alpha+\pi / 3\right) \\
f_{2}(x) & =f_{2}\left(x_{1}, x_{2}, \alpha\right)=\left(x_{1}+\frac{d \cos \alpha}{3 k}, x_{2}+\frac{d \sin \alpha}{3 k}, \alpha-\pi / 3\right) \\
f_{3}(x) & =f_{3}\left(x_{1}, x_{2}, \alpha\right)=\left(x_{1}+\frac{d \cos \alpha}{3 k}, x_{2}+\frac{d \sin \alpha}{3 k}, \alpha-\pi / 3\right) \\
f_{4}(x) & =f_{4}\left(x_{1}, x_{2}, \alpha\right)=\left(x_{1}+\frac{d \cos \alpha}{3 k}, x_{2}+\frac{d \sin \alpha}{3 k}, \alpha-3 \pi / 2\right) \\
g_{n}(x) & =n+1
\end{aligned}
$$



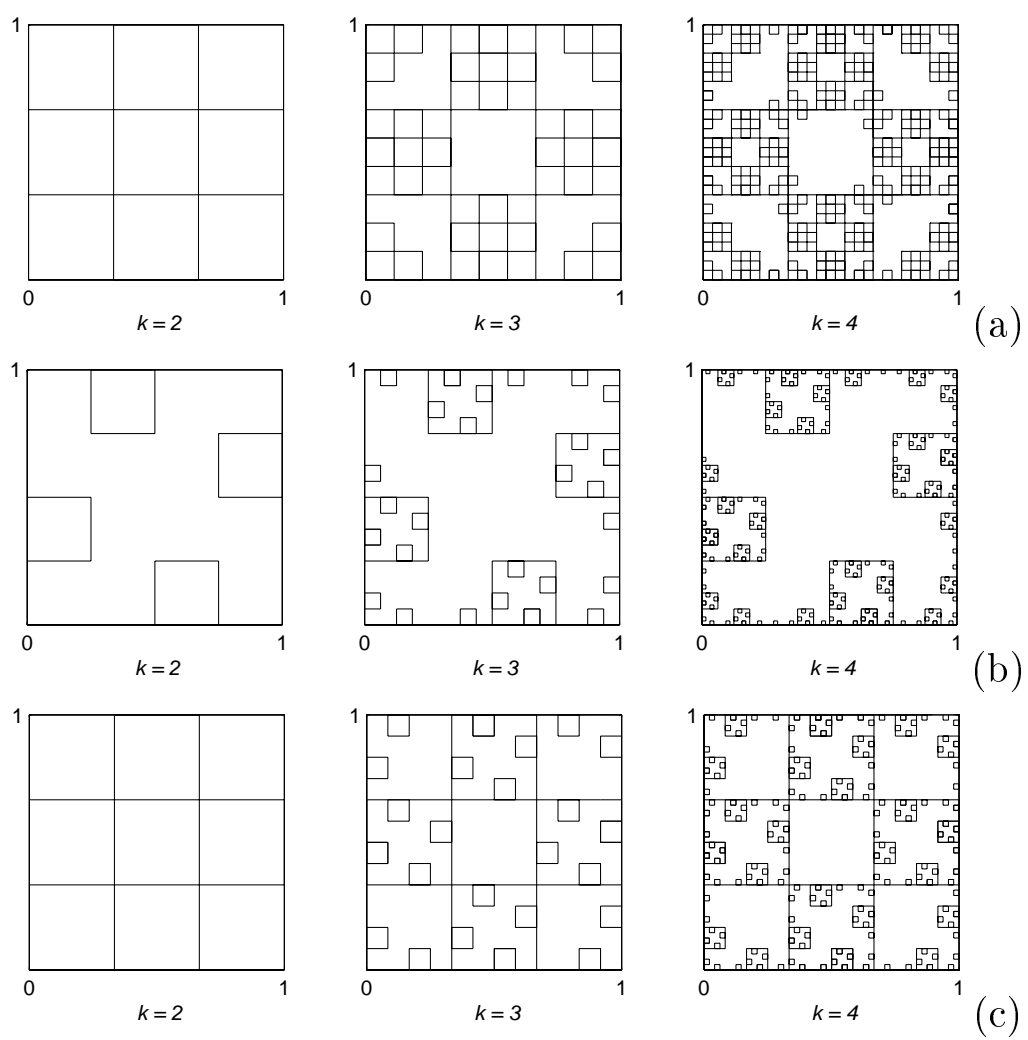

Figura 5.17: (a) Padrão geométrico produzido por um sistema que emula o L-sistema: $\Sigma=\{F,+,-\}, \omega_{0}=F+F+F+F, p: F \mapsto F+F-F F+F+F-F$ e $\delta=\pi / 2$. (b) Um padrão geométrico dado por um sistema de mapas funções de troca. (c) Funções de troca permitem produzir um padrão que "mistura" os padrões das figuras (a) e (b).

Encerrando esta seção, o sistema a seguir pretende representar um processo de difusão não uniforme em uma superfície, e é dado por:

$$
\begin{aligned}
F_{k}:(n, x) & \mapsto\left(g_{n}(x), f_{m}(x)\right) \\
f_{1}(x) & =f_{1}\left(x_{1}, x_{2}, \alpha\right)=\left(x_{1}+\frac{9}{10 k} d \cos \alpha, x_{2}+\frac{9}{10 k} d \sin \alpha, \alpha+0\right) \\
f_{2}(x) & =f_{2}\left(x_{1}, x_{2}, \alpha\right)=\left(x_{1}+\frac{9}{10 k} d \cos \alpha, x_{2}+\frac{9}{10 k} d \sin \alpha, \alpha+2 \pi / 3\right) \\
f_{3}(x) & =f_{3}\left(x_{1}, x_{2}, \alpha\right)=\left(x_{1}+\frac{9}{10 k} d \cos \alpha, x_{2}+\frac{9}{10 k} d \sin \alpha, \alpha-2 \pi / 5\right) \\
f_{4}(x) & =f_{4}\left(x_{1}, x_{2}, \alpha\right)=\left(x_{1}+\frac{9}{10 k} d \cos \alpha, x_{2}+\frac{9}{10 k} d \sin \alpha, \alpha+2 \pi / 3\right) \\
g_{n}(x) & =n+1
\end{aligned}
$$

As formas obtidas são mostradas na figura (5.16). As figuras (5.17) e (5.18) fornecem formas adicionais obtidas em simulações do modelo. 

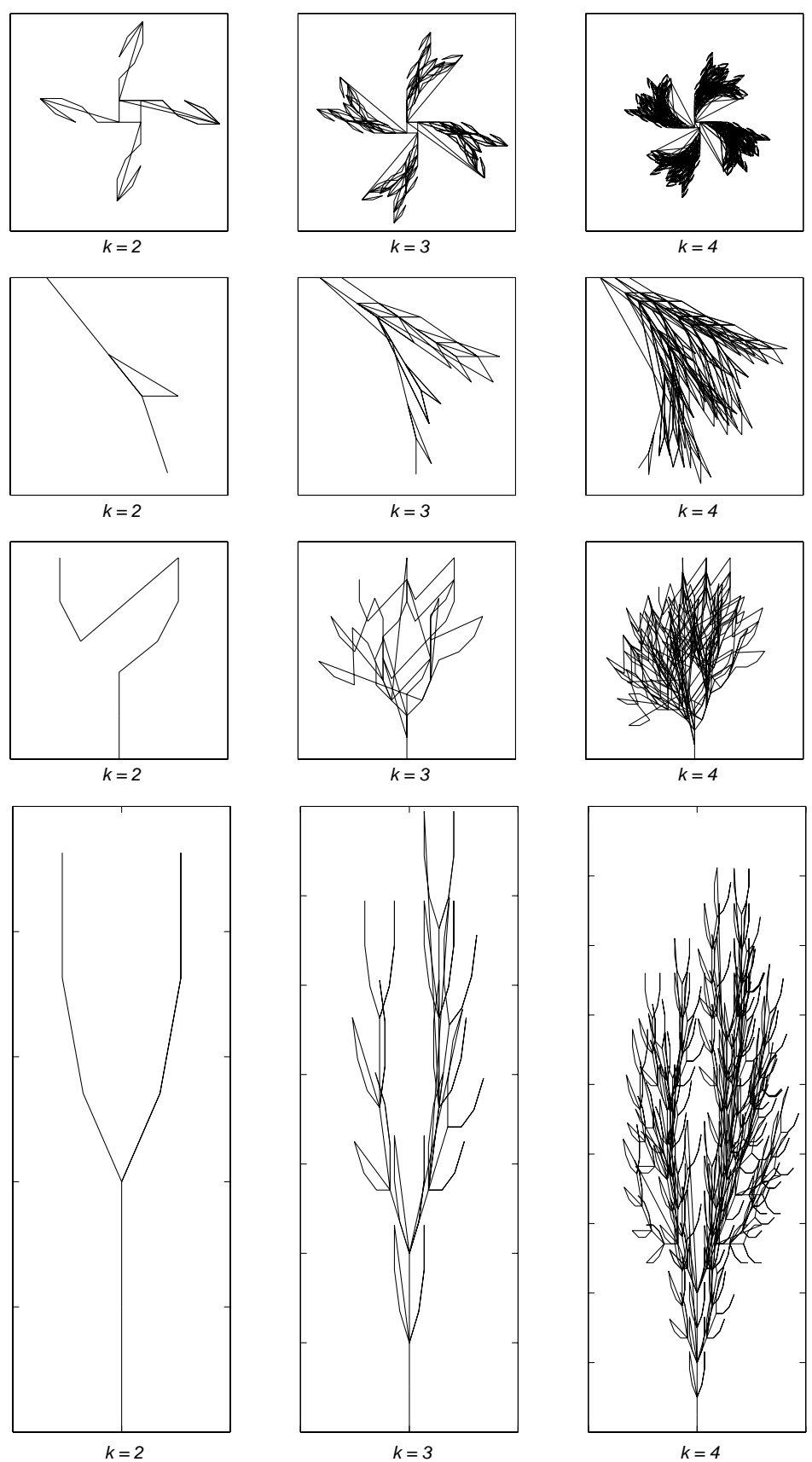

Figura 5.18: Padrões "botânicos" produzidos a partir de diferentes sistemas de mapas com funções de troca e que não possuem, de modo trivial, um L-sistema associado. 


\section{Mapas lineares $f: x \rightarrow A x+b$}

Por fim, empregamos, para construção de objetos gráficos, o uso de mapas lineares no plano, $f: x \rightarrow A x+b, x \in \mathbf{R}^{2}$, como operações elementares dos sistemas com funções de troca.

Transformações lineares são dadas por:

$$
f_{i}(x)=f_{i}\left(\begin{array}{l}
x_{1} \\
x_{2}
\end{array}\right)=\left(\begin{array}{ll}
a & b \\
c & d
\end{array}\right)\left(\begin{array}{l}
x_{1} \\
x_{2}
\end{array}\right)+\left(\begin{array}{l}
e \\
f
\end{array}\right)=A_{i} x+B_{i}
$$

sendo $a, b, c, d, e, f \in \mathbf{R}$.

Aqui, não requeremos que as transformações sejam contrações como nos sistemas de funções iteradas em geral.

Do mesmo modo que nos sistemas em que as operações elementares atuavam sobre coordenadas $\left(x_{1}, x_{3}, \alpha\right)$, as funções de troca permitem, aqui, alterar as operações lineares que são realizadas ao longo da evolução do sistema, ou com uma regra simples do tipo $g_{n}(x)=n+1$ (nesse caso, equivalente a um sistema de funções iteradas, ver anexo B) ou com regras que levam a diferentes operações elementares segundo o valor $x$.

Como exemplo, considere os conjuntos de transformações lineares (5.28) dados pelos valores $a, b, c, d, e, f \in \mathbf{R}$ em:

\begin{tabular}{ccccccc}
$f_{i, 1}$ & $\mathrm{a}$ & $\mathrm{b}$ & $\mathrm{c}$ & $\mathrm{d}$ & $\mathrm{e}$ & $\mathrm{f}$ \\
\hline$f_{1,1}$ & 0 & 0 & 0.5 & 0.5 & 0 & 0 \\
$f_{2,1}$ & 0.85 & 0.04 & -0.04 & 0.85 & 0 & 1.6 \\
$f_{3,1}$ & 0.2 & -0.26 & 0.23 & 0.22 & 0 & 1.6 \\
$f_{4,1}$ & -0.15 & 0.28 & 0.26 & 0.24 & 0 & 0.44
\end{tabular}

Tabela 5.4: Sistema de transformações lineares (IFS) para a produção do padrão da figura (5.19) [Barnsley, 1988].

\begin{tabular}{ccccccc}
$f_{i, 2}$ & $\mathrm{a}$ & $\mathrm{b}$ & $\mathrm{c}$ & $\mathrm{d}$ & $\mathrm{e}$ & $\mathrm{f}$ \\
\hline$f_{1,2}$ & 0 & 0 & 0.5 & 0.5 & 0 & 0 \\
$f_{2,2}$ & $\mathbf{0 . 9 5}$ & $\mathbf{0 . 6}$ & $\mathbf{- 0 . 6}$ & $\mathbf{0 . 9 5}$ & 0 & 1.6 \\
$f_{3,2}$ & $\mathbf{0 . 4}$ & -0.26 & 0.23 & 0.22 & 0 & 1.6 \\
$f_{4,2}$ & -0.15 & 0.28 & 0.26 & 0.24 & 0 & 0.44
\end{tabular}

Tabela 5.5: Sistema de transformações lineares modificado que produz o padrão da figura (5.20).

Cada um dos conjuntos das tabelas (5.4) e (5.5) gera um padrão gráfico (figuras (5.19) e (5.20)) empregando as funções $f_{i, \delta}$, como operações elementares, e $g_{n}(x)=n+1$ 


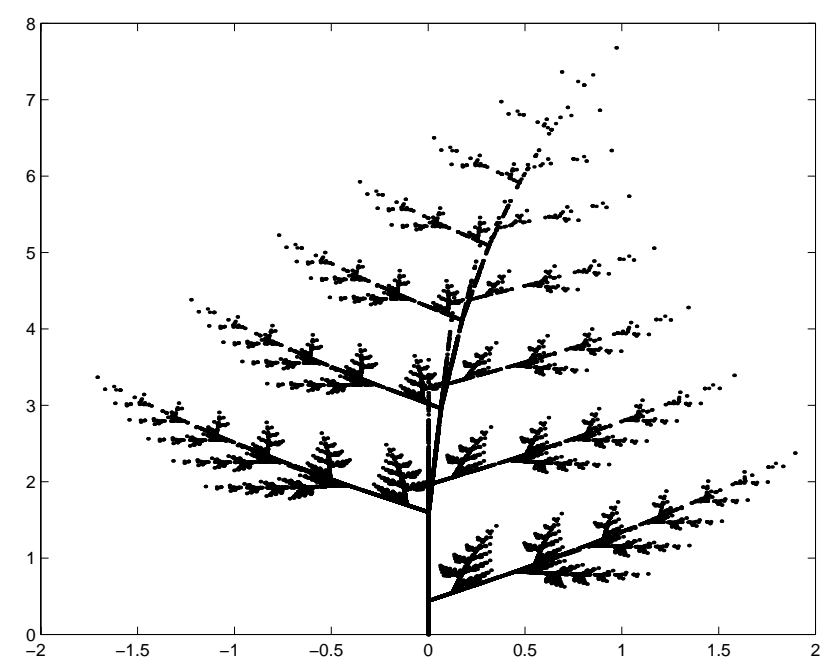

Figura 5.19: Estrutura fractal de planta, produzido pelas transformações lineares da tabela (5.4).

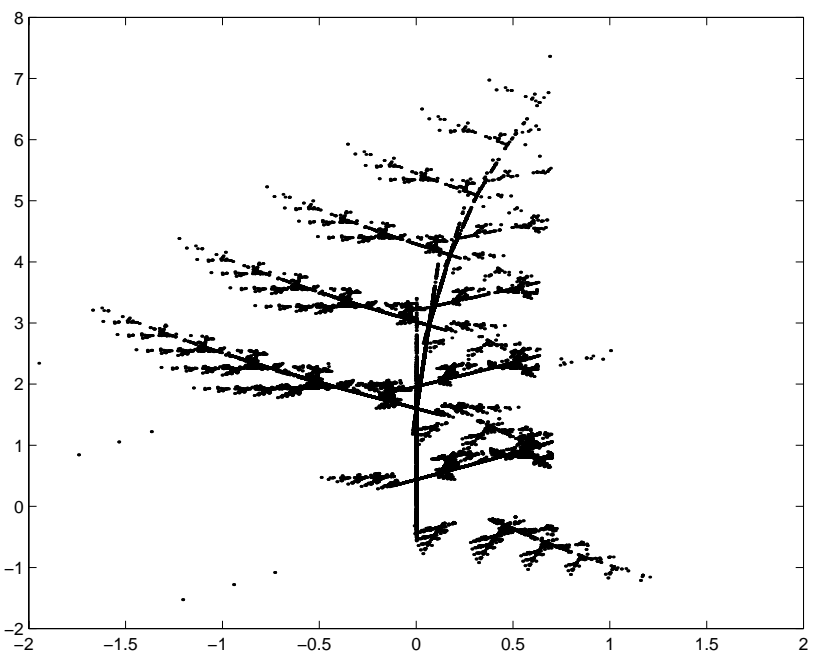

Figura 5.20: Estrutura fractal de planta, produzido pelas transformações lineares da tabela (5.5). 


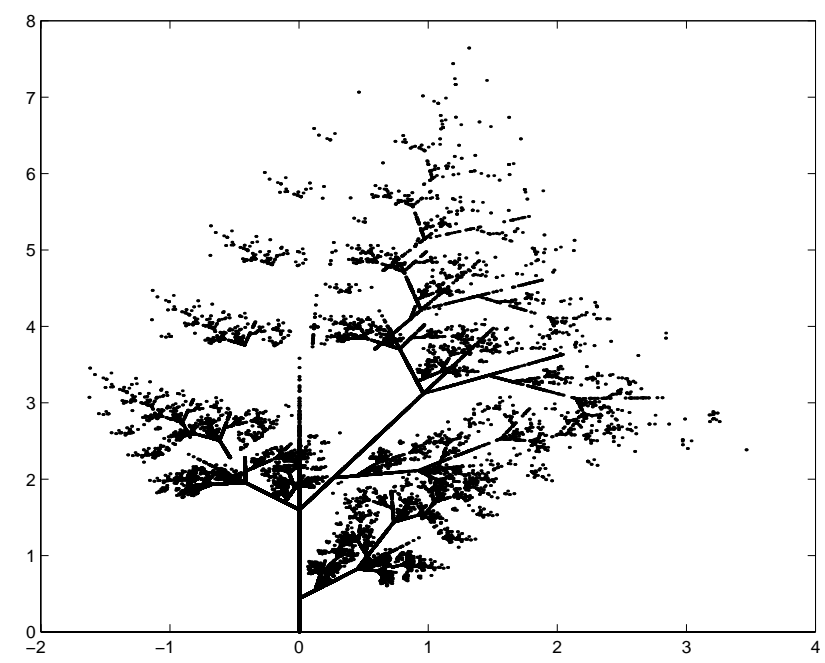

Figura 5.21: Estrutura fractal de planta, produzida pela combinação das transformações lineares das tabelas (5.4) e (5.5), com a função de troca (5.29).

como a função de troca. O uso de sistemas com funções de troca, entretanto, permite a composição de padrões mais elaborados. A figura (5.21) é produzida empregando-se os dois conjuntos anteriores de transformações, com a seguinte função de troca:

$$
g_{n}(x)=g_{n}\left(x_{1}, x_{2}\right)= \begin{cases}n+1, \delta=1 & \text { se } x_{1} \geq 3 \text { ou } x_{2} \geq 5 / 2 \\ n+1, \delta=2 & \text { se } x_{1}<3 \text { e } x_{2}<5 / 2\end{cases}
$$

De forma análoga produzimos as mutações sucessivas do triângulos de Sierpinski (figura (B.2)) originalmente produzido pelo seguinte conjunto de transformações lineares [Barnsley, 1988]:

\begin{tabular}{ccccccc}
$f_{i}$ & $\mathrm{a}$ & $\mathrm{b}$ & $\mathrm{c}$ & $\mathrm{d}$ & $\mathrm{e}$ & $\mathrm{f}$ \\
\hline$f_{1}$ & 0.5 & 0 & 0 & 0.5 & 1 & 1 \\
$f_{2}$ & 0.5 & 0 & 0 & 0.5 & 1 & 50 \\
$f_{3}$ & 0.5 & 0 & 0 & 0.5 & 50 & 50
\end{tabular}

Tabela 5.6: Sistema de transformações lineares modificado que produz o padrão da figura (5.21).

\subsection{Conclusão e sumário}

Neste penúltimo capítulo, discutimos a capacidade computacional de mapas. Fizemos uma revisão das principais abordagens sobre a computabilidade de mapas e realizamos uma série de simulações envolvendo modelos. Consideramos um último modelo, o de sistemas 

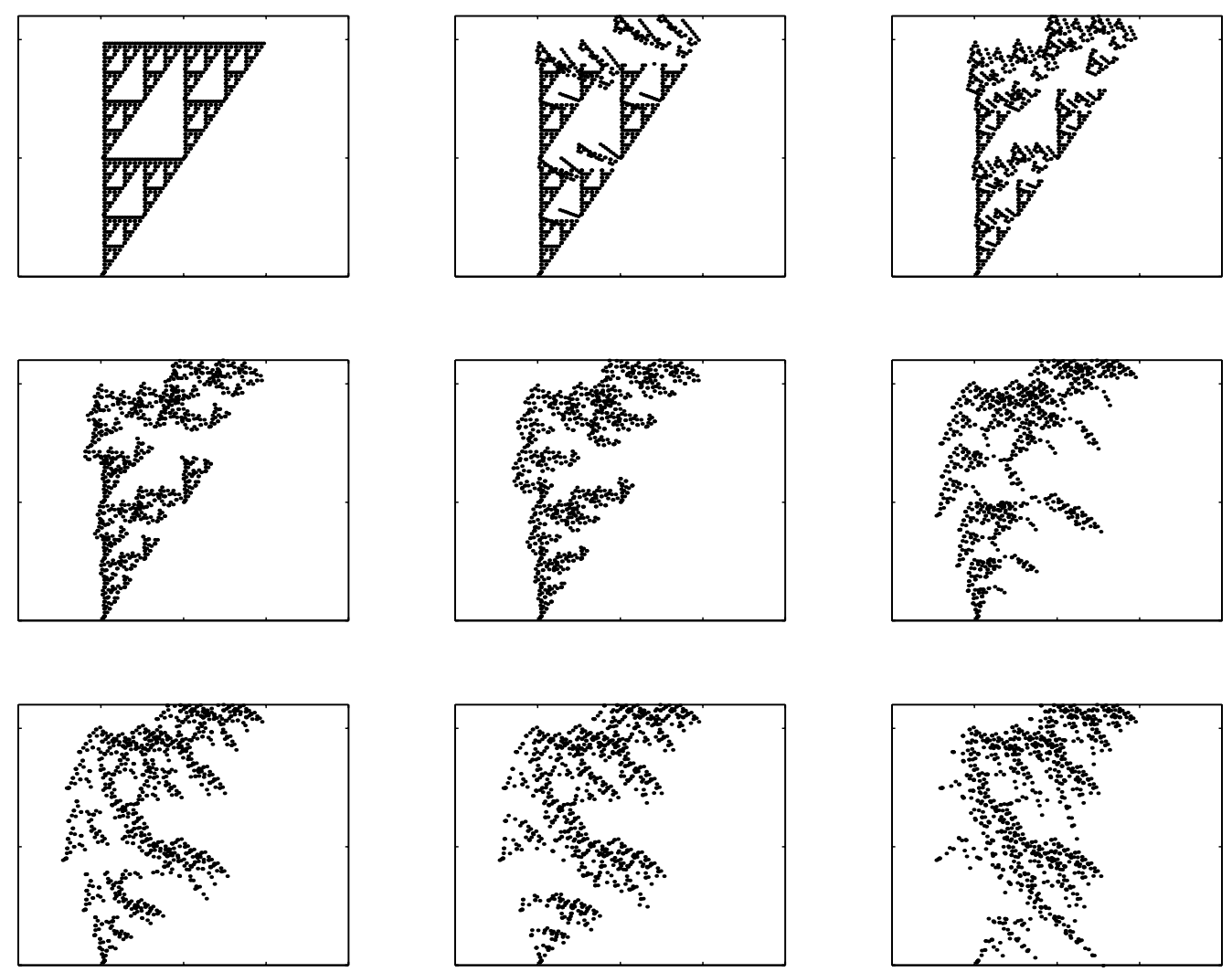

Figura 5.22: Triângulos de Sierpinski: mutações sucessivas por um sistema de mapas com funções de troca. 
de mapas com funções de troca, como aquele de maior interesse para novos modelos de computação e empregamos esses sistemas para a geração de imagens, obtendo resultados análogos aos produzidos por linguagens de processamento simbólico de imagens, como os L-sistemas, ou por sistemas de funções iteradas. Além disso, os sistemas de mapas com funções de troca nos pareceram mais flexíveis, permitindo a composição de diferentes conjuntos de transformações e, conseqüentemente, possibilitando a formação de novos padrões de objetos gráficos. O uso de sistemas, em que se associam probabilidades às funções de troca, deve permitir novos estudos, não só para produção de objetos gráficos, como para investigação de novos modelos de computação a valores reais. 


\section{Conclusão}

There is no "map"to human behaviour.

Björk

Aqui, consideramos a idéia de que se pode realizar o processamento de informação através de sistemas dinâmicos. Tal paradigma é a base de diversos sistemas, como aqueles baseados em redes neurais e autômatos celulares, para realização de tarefas computacionais. Dentre outros, três aspectos parecem justificar o estudo e o uso de sistemas dinâmicos para o processamento de informação: (a) pode-se tirar proveito de aspectos como paralelismo e tolerância a ruído e falhas, ausentes, de um modo geral, nos sistemas convencionais; (b) as limitações físicas dos sistemas convencionais [Frank, 2002]; (c) novos modelos de computação podem permitir a solução de problemas para os quais ainda não há algoritmos da computação convencional suficientemente eficientes ${ }^{1}$ [Blum et al., 1997] [Mertens, 2002]. Na abordagem aqui adotada, trajetórias e atratores de sistemas dinâmicos representam a lógica e os símbolos dos sistemas de computação convencionais. Muitas das propriedades computacionais desses sistemas, entretanto, permançem desconhecidas.

Aqui, empregamos sistemas estendidos de mapas para o processamento de informação. Esses sistemas podem exibir um grande número de comportamentos dinâmicos e propriedades de auto-organização e tolerância a ruído e falhas (perda de elementos). Sendo sistemas em tempo discreto são, ainda, mais adequados a implementações e simulações em sistemas computacionais. Neste trabalho foram, então, construídos modelos e realizadas simulações numéricas para o tratamento de três tarefas de natureza freqüentemente computacional: a análise de características geométricas de objetos gráficos; a agregação de elementos de forma auto-organizada; e a emulação de máquinas de Turing e linguagens.

\footnotetext{
${ }^{1}$ Referimo-nos aqui aos NP-problemas como o clássico problema do caixeiro viajante e a decisão da primalidade de um número.
} 


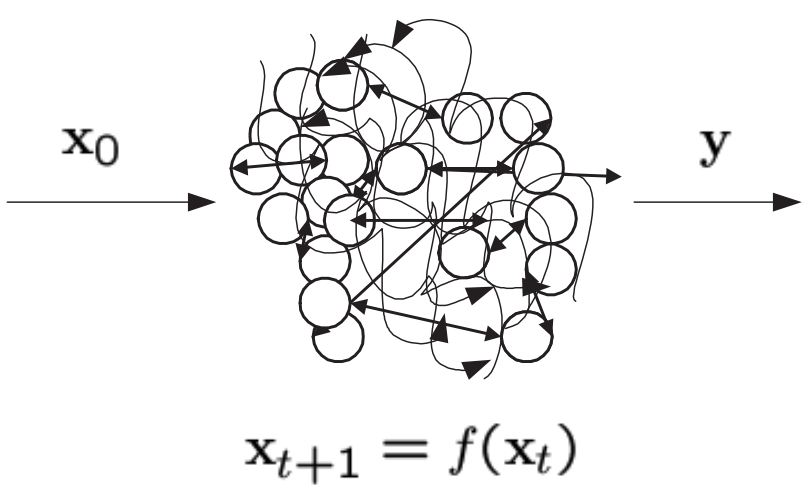

Figura 6.1: "Nosso" ponto de vista: sistemas dinâmicos provendo processamento da informação por múltiplos elementos conectados.

Principais contribuições desta tese: (1) Construímos um modelo de mapas acoplados para detecção de simetria de reflexão. Destaca-se, no modelo, a sua habilidade na detecção de simetria em cenas dinâmicas sem a necessidade de reinicialização de seus elementos. (2) Da análise de diferentes estruturas de acoplamento de mapas, mostramos que mapas acoplados em redes do tipo small-world podem apresentar um comportamento de auto-organização semelhante ao de redes regulares, com a formação de grupos de elementos sincronizados, mas com a vantagem de fornecerem uma topologia com menores caminhos médios entre seus elementos. (3) Por último, mostramos a capacidade de sistemas de mapas emularem qualquer máquina de Turing e, portanto, exibirem capacidade de processamento universal. Baseados em um modelo de computação que emprega mapas com funções de troca [Sato et al., 2000], desenvolvemos um sistema para a construção de imagens e padrões fractais, que, sob alguns aspectos, mostrou-se mais flexível e geral que os $L$-sistemas e os sistemas de funções iteradas.

Os aspectos aqui estudados levam-nos a uma série de outras questões, tendo, algumas delas, sido abordadas nos sumários dos capítulos anteriores. Ao término deste trabalho, contudo, destacamos alguns novos aspectos que devem ser objeto de nossos futuros estudos: (1) o estudo de valores críticos de coeficiente de agregação e menor caminho médio para sincronização de sistemas acoplados em topologias de rede small-world; (2) o uso de sistemas de mapas acoplados para realização de computação reversível; (3) o emprego de mapas com funções de troca para modelagem de sistemas biológicos como diferenciação celular; e (4) o uso desses sistemas para a construção de máquinas de Turing não deterministas. 


\section{Referências}

[Albert et al., 1999] Albert, R., Jeong, H., Barabási, A.L. "Diameter of the World Wide Web", Nature, 401, 130-131.

[Albert \& Barabási, 2002] Albert, R., Barabási, A.L. "Statistical mechanics of complex networks", Reviews of Modern Physics, 74, 47-96.

[Barábasi, 2002] Barabási, A.L. Linked: the new science of networks. Perseus Publishing.

[Barábasi \& Bonabeau, 2003] Barabási, A.L., Bonabeau, E. "Redes sem escala", Scientific American Brasil, junho/2003, 13, 64-72.

[Barahona \& Pecora, 2001] Barahona, M., Pecora, L.M. "Synchronization in small-world systems", Preprint cond-mat/0112023 em http://xxx.lanl.gov. Último acesso em: 05/10/2003.

[Barnsley, 1988] Barnsley, M.F. Fractals everywhere. Academic Press.

[Bar-Yam, 1997] Bar-Yam, Y. Dynamics of complex systems. Addison-Wesley.

[Blondel et al., 2000] Blondel, V.D., Bournez, O., Koiran, P., Tsitsiklis, J.N. "The stability of saturated linear dynamical systems is undecidable", Proceedings of STACS 2000, Lectures and Notes in Computer Science, 1770, 479-490.

[Blum et al., 1997] Blum, L., Schub, M., Cucker, F., Smale, S. Complexity and real computation. Springer-Verlag.

[Boccaletti et al., 2002] Boccaletti, S., Kurths, J., Osipov, G., Valladares, D.L., Zhou, C.S. "The synchronization of chaotic systems", Physics Reports, 366, 1101.

[Boffetta et al., 2002] Boffetta, G., Cencini, M., Falcioni, M., Vulpiani, A. "Predictability: A way to characterize complexity", Physics Reports, 356, 367-474.

[Bohland \& Minai, 2001] Bohland, J.W., Minai, A.A. "Efficient associative memory using small-world architecture", Neurocomputing, 38, 489-496.

[Brunde \& Havlin, 1995] Brunde, A., Havlin, S. Fractals and disordered systems. Springer, 2nd ed.

[Camazine et al., 2001] Camazine, S., et. al. Self-organization in biological systems. Princeton University Press.

[Chen \& Wang, 2002] Chen, K., Wang, D.L., "A dynamically coupled neural oscillator network for image segmentation", Neural Networks, 15, 423-439. 
[Campbell \& Wang, 1996] Campbell, S., Wang, D. "Synchronization and desynchronization in a network of locally coupled Wilson-Cowan oscillators", IEEE Trans. Neural Networks, 7, 541-554.

[Crawford, 1991] Crawford, J. D. "Introduction to bifurcation theory", Reviews of Modern Physics, 63, 4, 991-1037.

[Cross, 2003] Cross, M. "Chaos on the Web Physics 161: Introduction to Chaos", software em http://www.cmp.caltech.edu/ mcc/Chaos_Course/

Lesson14/Introduction.html. Último acesso em: 05/10/2003.

[Del-Moral, 2001] Del-Moral-Hernandez, E. "Studying neural networks of bifurcating recursive processing elements: quantitative methods for architecture, design and performance analysis", Lecture Notes in Computer Science, 2084, 546553.

[Del-Moral, 2003] Del-Moral-Hernandez, E. "Neural networks with chaotic recursive nodes: techniques for design of associative memories, contrast with Hopfield architectures, and extensions for time-dependent inputs", Neural Networks, $16,675-682$.

[Devaney, 1989] Devaney, R. An introduction to chaotic dynamical systems. AddisonWesley, 2nd ed.

[Dorogovtsev \& Mendes, 2002] Dorogovtsev, S.N., Mendes, J.F.F. "Evolution of networks", Advances in Physics, 51, 1079-1187.

[Frank, 2002] Frank, M.P. "The physical limits of computing", Computing in science and engineering, may/june issue, 16-26.

[Ferrara \& Prado, 1994] Ferrara, N.F., Prado, C.P.C. Caos, uma introdução. Ed. Edgard Blucher.

[Freeman, 1992] Freeman, W.J. "Tutorial on neurobiology: From single neurons to brain chaos", International Journal of Bifurcation and Chaos, 2, 451-452.

[Gade \& Hu, 2000] Gade, P.M., Hu, C.-K. "Synchronous chaos in coupled map with small-world interactions", Pysical Review E, 62, 6409-6413.

[Gelover et al., 2000] Gelover-Santiago, A.L., Lima, R., Martínez-Mekler, G. "Synchronization and cluster periodic solutions in globally coupled maps", Physica A, 283, 131-135.

[Gramß et al., 1998] Gramß, T., Bornholdt, S., GroB, M., Mitchell, M., Pellizzari, T. Non-standard computation: molecular computation - cellular automata evolutionary algorithms - quantum computers. Wiley-VCR.

[Guckenheimer \& Holmes, 1983] Guckenheimer, J., Holmes, P. Nonlinear oscillations, dynamical systems, and bifurcations of vector fields. Springer-Verlag.

[Haberman, 1997] Haberman, R. Elementary applied partial differential equations: with Fourier series and boundary value problems. Prentice Hall, 3nd ed. 
[Hassoun, 1993] Hassoun, M.H. eds. Associative neural memories: theory and implementation. Oxford University Press.

[Hayashi, 1994] Hayashi, Y. "Oscillatory neural network and learning of continuously transformed patterns", Neural Networks, 7, 219-231.

[Haykin, 1999] Haykin, S. Neural networks: a comprehensive foundation. Prentice Hall, 2nd ed.

[He et al., 1999] He, Z., Zhang, Y., Yang, L. "The study of chaotic neural network and its applications in associative memory", Neural Processing Letters, 9, 163-175.

[Hinton \& Sejnowski, 1999] Hinton, G., Sejnowski, T.J. eds. Unsupervised learning: foundations of neural computation. MIT Press.

[Holmgren, 1996] Holmgren, R.A. A first course in discrete dynamical systems. SpringerVerlag, 2nd ed.

[Hopcroft \& Ullman, 1979] Hopcroft, J.E., Ullman, J.D. Introduction to automata theory, languages and computation. Addison-Wesley.

[Hopfield, 1982] Hopfield, J.J. "Neural networks and physical systems with emergent collective computational abilities", Proceedings of National Academy of Sciences, 79, 2554-2558.

[Hoppensteadt, 1997] Hoppensteadt, F.C. An introduction to the mathematics of neurons : modeling in the frequency domain. Cambridge University Press, 2nd ed.

[Ito, 2000] Ito, J., Kaneko, K. "Self-organized hierarchical structure in a plastic network of chaotic units", Neural Networks, 13, 275-281.

[Kaneko, 1984] Kaneko, K. "Period-doubling of kink-antikink patterns, quasi-periodicity in antiferro-like structures and spatial intermittency in coupled map lattices - toward a prelude to a field theory of chaos", Prog. Theor. Phys. 72, 480486.

[Kaneko, 1992] Kaneko, K. "Overview of coupled map lattices", International Journal of Bifurcation and Chaos, 2(3), 279-282.

[Kaneko, 1993] Kaneko, K. "The coupled map lattice: introduction, phenomenology, Lyapunov analysis, thermodynamics and applications", In: Theory and applications of coupled map lattices, 1-49. Wiley.

[Kaneko, 1994] Kaneko, K. "Relevance of dynamic clustering to biological networks", Physica D, 75, 55-73.

[Kaneko, 1997] Kaneko, K. "Coupled maps with growth and death: an approach to cell differentiation", Physica D, 103, 505-527.

[Kaneko \& Tsuda, 2000] Kaneko, K., Tsuda, I. Complex systems: chaos and beyond. Springer-Verlag.

[Kaplan \& Glass, 1995] Kaplan, D., Glass, L. Understanding nonlinear dynamics. Springer-Verlag. 
[Kimura \& Nakano, 1998] Kimura, M., Nakano, R. "Learning dynamical systems by recurrent neural networks from orbits", Neural Networks, 11, 1589-1599.

[Kleinberg, 2000] Kleinberg, J.M. "Navigation in a small world", Nature, 406, 845-845.

[Koiran et al., 1994] Koiran, P., Cosnard, M., Garzon, M. "Computability with lowdimensional dynamical systems", Theorical Computer Science, 132, 113128.

[Konen \& Malsburg, 1993] Konen, W., Malsburg, C.v.d. "Learning to generalize from single examples in the dynamic link architecture", Neural Computation, 5, 719-735.

[Lago et al., 2000] Lago-Fernandez, L.F., Huerta, R., Corbacho, F., Sigëenza, J.A. "Fast response and temporal coherent oscillations in small-world networks", Physical Review Letters, 84, 2758-2761.

[Lorenz, 1963] Lorenz, E.N. "Deterministic non-periodic flow", J. Atmos. Sci., 20, 130141.

[Lucchesi et al., 1979] Lucchesi, C.L., Simon, I.(Imre), Simon, I.(Istvan), Simon, J., Kowaltowski, T. Aspectos teóricos da computação. Impa.

[Maza et al., 2000] Maza, D., Boccaletti, S., Mancini, H. "Phase clustering and collective behaviors in globally coupled map lattices due to mean field effects", International Journal of Bifurcation and Chaos, 10, 829-833.

[Menezes, 2001] Menezes, M.A.F. "Transições de fase geométricas em redes esparsas e simulações de um processo de não-equilíbrio", Tese de doutorado do Instituto de Física da Universidade Federal Fluminense. Orientador: Penna, T.J.P.

[Mertens, 2002] Mertens, S. "Computational complexity for physicists", Computing in science and engineering, may/june issue, 31-46.

[Minsky, 1967] Minsky, M. Computation: finite and infinite machines. Prentice-Hall.

[Monteiro, 2002] Monteiro, L.H.A. Sistemas dinâmicos. Ed. Livraria da Física.

[Monteiro et al., 2002] Monteiro, L.H.A., Bussab, M.A., Chaui-Berlinck, J.G. "Analytical results on Wilson-Cowan neuronal network modified model", Journal of Theorical Biology, 219, 83-91.

[Monteiro et al., 2003] Monteiro, L.H.A., Canto, N.C.F., Chaui-Berlinck, J.G., Orsatti, F.M., Piqueira, J.R.C. "Global and partial synchronism in phase-locked loop networks", IEEE Trans. on Neural Networks (to appear).

[Moore, 1990] Moore, C. "Unpredictability and undecidability in dynamical systems", Physical review letters, 64(20), 2354-2357.

[Moyano et al., 2001] Moyano, L.G., Abramson, G., Zanette, D.H. "Synchronization learning of coupled chaotic maps", European Physical Journal B, 22, 223228 . 
[Newman \& Watts, 1999] Newman, M.E.J., Watts, D.J. "Scaling and percolation in the small-world network model", Physical Review E, 60, 7332-7342.

[Newman, 2000] Newman, M.E.J. "Models of the small world: a review", Journal of Statistical Physics, 101, 819-841.

[Nishii, 1998] Nishii, J. "The learning model goes oscillatory networks", Neural Networks, $11,249-257$.

[Olien, 1997] Olien, L., Bélair, J. "Bifurcations, stability, and monotonicity properties of a delayed neural network model", Physica D, 102, 349-367. Elsevier.

[Oliveira, 2000] Oliveira, R. "Redes neurais recorrentes com aprendizado no espaço de fases", Tese de mestrado em Engenharia Elétrica da Universidade Presbiteriana Mackenzie. Orientador: Monteiro, L.H.A.

[Oliveira \& Monteiro, 2000] Oliveira, R., Monteiro, L.H.A. "Atratores contínuos em redes recorrentes com aprendizado no espaço de fases", IEEE Proceedings of VIth Brazilian Symposium on Neural Networks, SBRN'2000, CD.

[Oliveira \& Monteiro, 2002a] Oliveira, R., Monteiro, L. H. A. "Symmetry detection using global-locally coupled maps", Proceedings of International Conference on Artificial Neural Networks 2002, ICANN, Lecture Notes in Computer Science, 2415, 75-80.

[Oliveira \& Monteiro, 2002b] Oliveira, R., Monteiro, L. H. A. "Uso de uma rede de mapas acoplados para detecção de simetria", Revista Mackenzie de Engenharia e Computação, 3, 119-131.

[Oliveira \& Monteiro, 2003] Oliveira, R., Monteiro, L. H. A. "Clustering in coupled maps on small-world networks", Proceedings of the International Joint Conference on Neural Networks 2003, IJCNN, 1, 337-340.

[Orponen \& Matamala, 1996] Orponen, P., Matamala, M. "Universal computation by finite two-dimensional coupled map lattices", Proceedings of 4th Workshop on Physics and Computation, PhyComp96, 243-247.

[Pajek, 2003] Pajek: Batagelj, V., Mrvar, A. "Pajek - Program for Large Network Analysis", software em http://vlado.fmf.uni-lj.si/pub/networks/pajek/. Último acesso em: 05/10/2003.

[Peitgen et al., 1992] Peitgen, H.O., Jürgens, H., Saupe, D. Fractals for the classroom, v.2 Complex systems and mandelbrot set. Springer-Verlag.

[Pickover,1990] Pickover, C.A. Computers, pattern, chaos, and beauty: graphics from an unseen world. Dover ed. in 2001.

[Pikovsky et al., 2001] Pikovsky, A., Rosenblum, M., Kurths, J. Synchronization: A universal concept in nonlinear sciences. Cambridge University Press.

[Popovych et al., 2002] Popovych, O., Pikovsky, A., Maistrenko, Yu. "Cluster-splitting bifurcation in a system of coupled maps", Physica D, 168-169, 106-125. Elsevier. 
[Prusinkiewicz \& Hanan, 1989] Prusinkiewicz, P., Hanan, J. Lindenmayer systems, fractals, and plants. Springer-Verlag, 2nd ed.

[Rosen, 1995] Rosen, J. Symmetry in science: an introduction to the general theory. Springer-Verlag.

[Saito \& Kaneko, 2001] Saito, A., Kaneko, K. "Inaccessibility and undecidability in computation, geometry, and dynamical systems", Physica D, 155, 1-33.

[Sato et al., 2000] Sato, Y., Taiji, M., Ikegami, T. "On the power of nonlinear mappings in switching map systems", Unconventional Models of Computation, UMC2K (Springer-Verlag), 234-247.

[Sejnowski et al., 1986] Sejnowski, T.J., Keinker, P.K., Hinton, G.E. "Learning symmetry groups with hidden units: Beyhond the perceptron", Physica D, 22, 260275 .

[Shen et al., 1999] Shen, D., IP, H.H.S., Cheung, K.K.T., Teoh, E.K. "Symmetry detection by generalied complex (GC) moments: A close-form solution", IEEE Trans. Pattern Analysis and Machine Intelligence, 21, 466-476.

[Siegelmann \& Sontag, 1991] Siegelmann, H.T., Sontag, E.D. "Turing compatibility with neural nets", Applied Mathematics Letters, 4, 77-80.

[Solé et al., 2003] Solé, R.V., Cancho, R.F., Montoya, J.M., Valverde, S. "Selection, tinkering, and emergence in complex networks", Complexity, 8(1), 20-33.

[Sontag, 1996] Sontag, E.D. "Recurrent neural networks: some learning and systemstheoretic aspects", Technical report, Department of Mathematics, Rutgers University em http://gubbio.cs.berkeley.edu/mlpapers. Último acesso em: $05 / 10 / 2003$.

[Strogatz \& Stewart, 1993] Strogatz, S.H., Stewart, I. "Coupled oscillators and biological synchronization", Scientific American, December (1993), 102-108.

[Strogatz, 1994] Strogatz, S.H. Nonlinear dynamics and chaos: with applicactions to physics, biology, chemistry, and engineering. Persus Books Publishing.

[Strogatz, 2001] Strogatz, S.H. "Exploring complex networks", Nature, 410, 268-276.

[Wang, 1991] Wang, X. "Period-doublings to chaos in a simple neural network: an analytical proof", Complex Systems, 5, 425-441.

[Wang \& Chen, 2002] Wang, X.F., Chen, G. "Synchronization in small-world dynamical networks", International Journal of Bifurcation and Chaos, 12, 187-192.

[Wang \& Chen, 2002b] Wang, X.F., Chen, G. "Synchronization in scale-free dynamical networks: robustness and fragility", IEEE Transactions on Circuits and Systems-I: Fundamental Theory and Applications, 49, 54-61.

[Wang \& Chen, 2003] Wang, X.F., Chen, G. "Complex networks: small-world, scale-free and beyond", IEEE Circuits and Systems Magazine, 1Q2003, 6-20. 
[Watts \& Strogatz, 1998] Watts, D.J., Strogatz, S.H. "Collective dynamics of small-world networks", Nature, 393, 440-442.

[Watts, 1999] Watts, D.J. Small worlds: the dynamic of networks between order and randomness. Princeton University Press.

[Wei \& Jia, 2002] Wei, G.W., Jia, Y.Q. "Synchronization-based image edge detection", Europhys. Lett., 59, 814-819.

[Wei \& Ruan, 1999] Wei, J., Ruan, S. "Stability and bifurcation in a neural network model with two delays", Physica D, 130, 255-272.

[White \& Houseman, 2003] White, D.R., Houseman, M. "The navegability of strong ties: small worlds, tie strength, and network topology", Complexity, 8, 72-81.

[Wilson \& Cowan, 1972] Wilson, H.R, e Cowan, J.D. "Excitatory and inhibitory interactions in localized populations of model neurons", Biophysical Journal, 12, $1-24$.

[Wolfram, 2001] Wolfram, S. A new kind of science. Wolfram Media, Inc.

[Zamir, 2001] Zamir, M. "Arterial branching within the confines of fractal L-system formalism", Journal Gen. Physiol. 118, 267-275.

[Zhao et al., 2000] Zhao, L., Macau, E.E.N., Omar, N. "Scene segmentation of the chaotic oscillator network", International Journal of Bifurcation and Chaos, 10, 1697-1708.

[Zhao, 2000b] Zhao, L. "A dynamically coupled chaotic oscillatory correlation network", IEEE Proceedings of VIth Brazilian Symposium on Neural Networks, SBRN'2000, 66-71.

[Zhao \& Macau, 2001] Zhao, L., Macau, E.E.N. "A network of dynamically coupled chaotic maps for scene segmentation", IEEE Transactions on neural networks, 12, 1375-1385. 


\section{Anexo A - Autômatos celulares}

Apresentam-se, a seguir, conceitos básicos de autômatos celulares.

Autômatos celulares são sistemas constituídos de um grande número de elementos simples e idênticos com conectivade local entre seus elementos. Esses sistemas compartilham uma série de propriedades com sistemas de mapas acoplados. Eles possuem capacidade de computação universal, podem apresentar dinâmicas complexas e auto-organização de seus elementos, e são empregados no estudo de problemas de difusão, percolação, termodinâmica, pigmentação, de transporte etc. [Wolfram, 2001].

Um autômato celular consiste de dois componentes: um espaço celular e uma regra de transição. O espaço celular é uma malha de $N$ máquinas de estados finitos (células), sendo $\Sigma$ o conjunto dos $k$ estados possíveis de cada célula e $s_{i}(t)$ o estado do $i$-ésimo elemento no instante $t$. Todas as célula possuem um padrão idêntico de conectividade com outras células. As células conectadas a um elemento constituem a sua vizinhança e os estados do elemento $i$ e de seus vizinhos no instante $t$ é representado por $\eta_{i}(t)$.

A regra de transição $\phi\left(\eta_{i}(t)\right)$ fornece a atualização dos estados $s_{i}(t) \mapsto s_{i}(t+1)$ em função dos estados de cada elemento e dos seus vizinhos. Tipicamente, a cada iteração, todas as células têm seus estados atualizados pela regra $\phi\left(\eta_{i}(t)\right)$ sincronamente ${ }^{1}$.

Autômato celular unidimensional. Nas tabelas (A.1) e (A.2), apresenta-se um autômato celular unidimensional com $\Sigma=\{0,1\}$ e o conjunto de elementos, $i-1, i$ e $i+1$, definindo a vizinhança da célula $i$. Nas figuras (A.1) e (A.2), simulamos a evolução de estados de autômatos celulares unidimensionais para diferentes regras (os zeros correspondem aos valores brancos).

\footnotetext{
${ }^{1}$ No mesmo instante.
} 


$\begin{array}{ccccccccc}\eta_{i} & 000 & 001 & 010 & 011 & 100 & 101 & 110 & 111 \\ \phi\left(\eta_{i}\right) & 1 & 0 & 0 & 1 & 0 & 1 & 0 & 1\end{array}$

Tabela A.1: Regra de transição $\phi$. A seqüência da segunda linha, considerada como um número binário de 8 dígitos, fornece o número de Wolfram da regra. Acima, a regra 149.

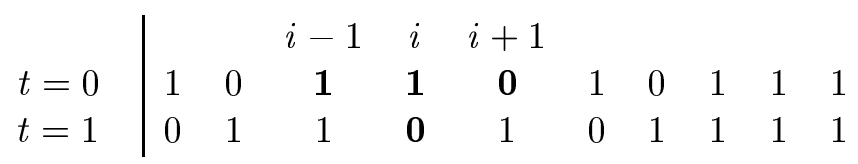

Tabela A.2: Atualização dos estados do autômato celular pela regra 149 (tabela A.1). Aplica-se condição de contorno periódica (o primeiro elemento é vizinho do último). Destaca-se a vizinhança do quarto elemento e seu novo valor após a aplicação da regra de transição.

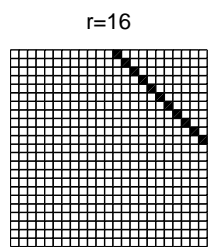

$r=72$

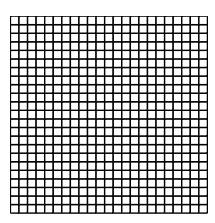

$r=96$

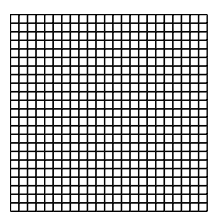

$r=128$

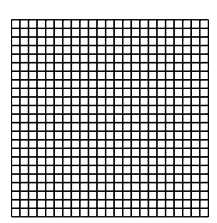

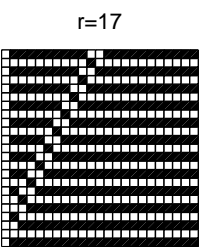

$r=73$

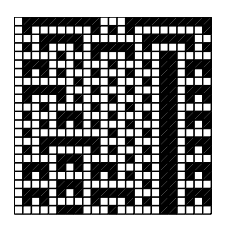

$r=97$

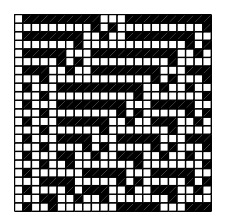

$r=129$

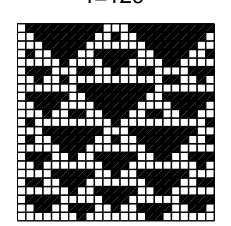

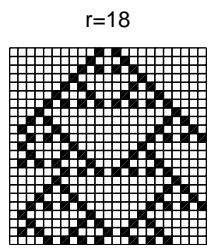

$r=74$

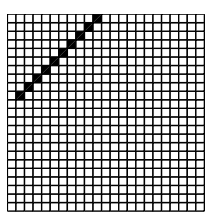

$r=98$

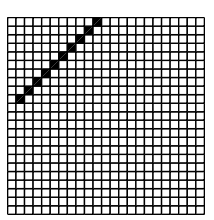

$r=130$

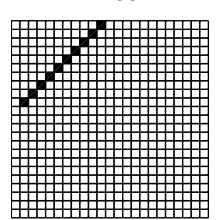

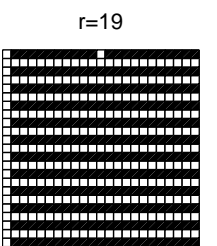

$r=75$

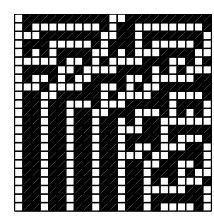

$r=99$

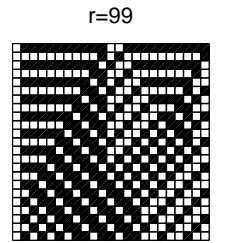

$r=131$

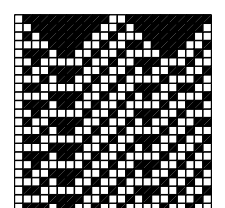

Figura A.1: Exemplos de padrões gerados por automatos celulares, a partir de diferentes condições iniciais e diferentes regras $r$. Cada linha corresponde à configuração do autômato em um instante $t$ cujos estados evoluem verticalmente, de cima para baixo. 

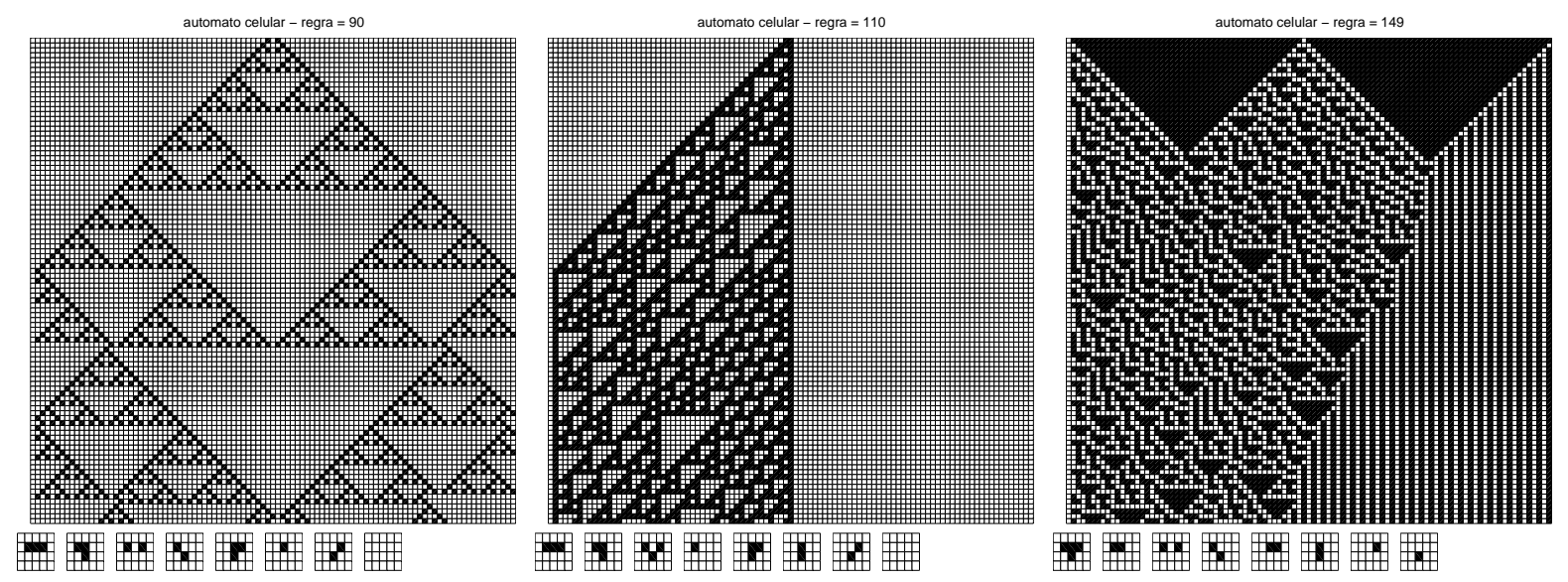

Figura A.2: Três exemplos de automatos celulares: a regra 90 que exibe um padrão semelhante ao dos fractais de Sierpinski [Brunde \& Havlin, 1995]; a regra 110 que possui capacidade de realizar computação universal [Wolfram, 2001]; e a regra 149 que exibe um padrão semelhante ao encontrado em algumas conchas de moluscos [Wolfram, 2001]. 


\section{Anexo B - IFS, sistemas de funções iteradas}

Sistemas de funções iteradas (iteraded function systems ou IFS's) são uma forma usual de construção de fractais e surgem em aplicações de codificação de imagens e geração de padrões fractais.

Um sistema (hiperbólico) ${ }^{1}$ de funções iteradas consiste de um conjunto finito de mapas de contração $f_{i}: X \mapsto X, n=1, \ldots, N$ e $(X, d)$ um espaço métrico completo com norma d. Esse sistema é, então, representado por $F=\left\{f_{1}, \ldots, f_{i}\right\}$.

Um mapa, $f_{i}: X \mapsto X$, é denominado de contração ou, simplesmente, uma contração, se existe uma constante $c$ tal que $d\left(f_{i}(x), f_{i}(y)\right) \leq c d(x, y)$ para todo $x, y \in X$. Por espaço métrico completo, entende-se aquele em que toda seqüência de Cauchy de $X$ converge para um valor também de $X$.

O IFS $F$ pode ser estendido para todos subconjuntos não vazios e limitados de $X$. Para tanto, define-se:

$$
f_{i}(A)=\left\{f_{i}(x): x \in A \subset X\right\}
$$

e

$$
S(A)=\bigcup_{i=1}^{\infty} f_{i}(A)
$$

Seja um conjunto $A \subset X$. Denotam-se as $n$ sucessivas aplicações de $S$ sobre a $A$ por $S^{n}(A)$, isto é, $S^{0}(A)=A$ e $S^{n}(A)=S^{n-1}(A)$. Se $F$ é então um conjunto de contrações, denomina-se o atrator de $F$, o conjunto A definido por:

$$
\mathbf{A}=\lim _{n \rightarrow \infty} S^{n}(A)
$$

\footnotetext{
${ }^{1} \mathrm{Na}$ prática, o termo IFS é muitas vezes é empregado para designar apenas um conjunto finito de mapas que atuam sobre um espaço métrico sem condições particulares sobre os mapas. Dessa forma, omite-se, em geral o termo hiperbólico.
} 
A expressão (B.3) fornece um método de se obter o atrator A aplicando-se iterativamente todas as possíveis seqüências de mapas de $F^{2}$. Na prática, o atrator é construído empregando-se um número finito de iterações.

Aqui, é suficiente considerar IFS definidos no plano $\mathbf{R}^{2}$, com a métrica euclidiana, e contrações lineares no plano como translações, rotações, reduções de escala e suas combinações, as quais são expressas por:

$$
f_{i}\left(\begin{array}{l}
x \\
y
\end{array}\right)=\left(\begin{array}{ll}
a & b \\
c & d
\end{array}\right)\left(\begin{array}{l}
x \\
y
\end{array}\right)+\left(\begin{array}{l}
e \\
f
\end{array}\right)
$$

sendo $a, b, c, d, e, f \in \mathbf{R}$ e $a, b, c, d<1$ (contrações).

Triângulo de Sierpinski. O triângulo de Sierpinski é uma forma de triângulos inseridos em outros (ver figura B.1), e surge na construção do triângulo de Pascal e na evolução de sistemas de autômatos celulares [Pickover,1990]. Ele também aparece como o atrator do IFS dado pelo seguinte conjunto de contrações no plano:

$$
\begin{aligned}
f_{1}\left(\begin{array}{l}
x \\
y
\end{array}\right) & =\left(\begin{array}{cc}
0.5 & 0 \\
0 & 0.5
\end{array}\right)\left(\begin{array}{l}
x \\
y
\end{array}\right)+\left(\begin{array}{l}
0 \\
0
\end{array}\right) \\
f_{2}\left(\begin{array}{l}
x \\
y
\end{array}\right) & =\left(\begin{array}{cc}
0.5 & 0 \\
0 & 0.5
\end{array}\right)\left(\begin{array}{l}
x \\
y
\end{array}\right)+\left(\begin{array}{c}
0.5 \\
0
\end{array}\right) \\
f_{3}\left(\begin{array}{l}
x \\
y
\end{array}\right) & =\left(\begin{array}{cc}
0.5 & 0 \\
0 & 0.5
\end{array}\right)\left(\begin{array}{l}
x \\
y
\end{array}\right)+\left(\begin{array}{c}
0 \\
0.5
\end{array}\right)
\end{aligned}
$$

\footnotetext{
${ }^{2}$ A prova da existência desse atrator baseia-se teorema do ponto fixo de Banach. Esse e outros teoremas sobre IFS podem ser encontrados em [Barnsley, 1988].
} 

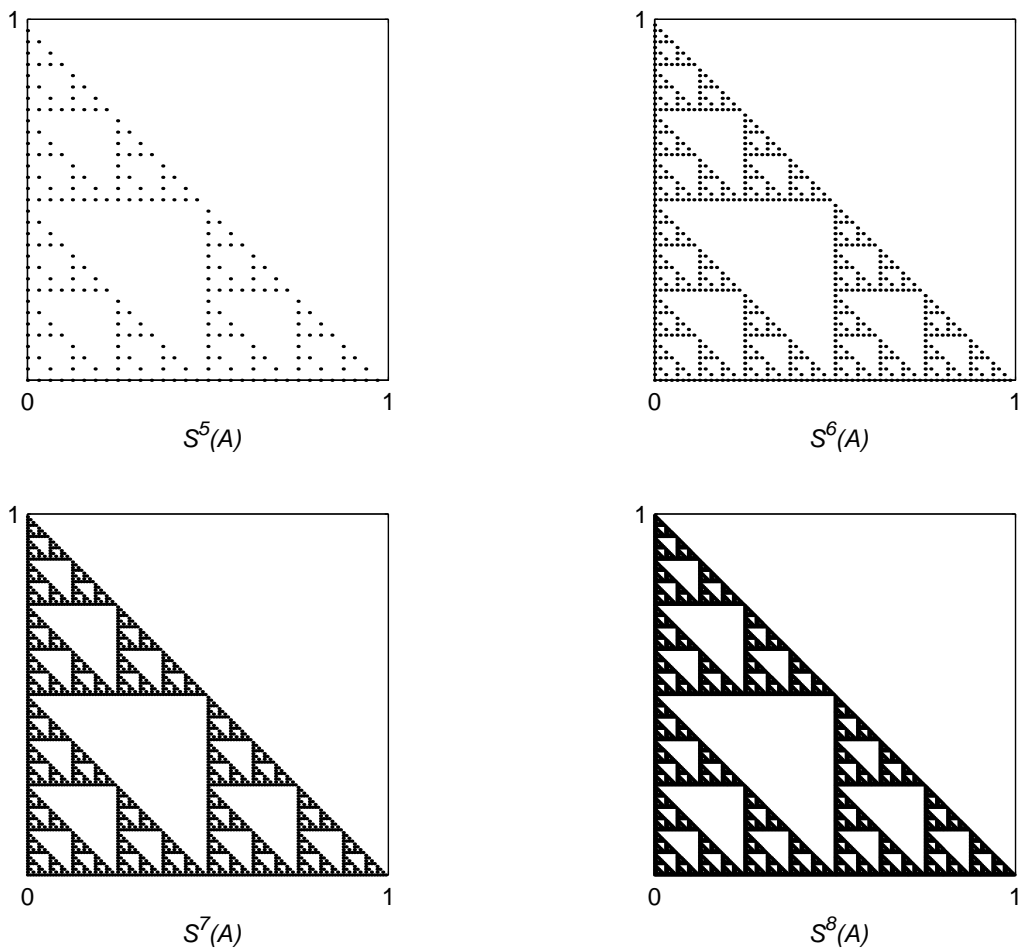

Figura B.1: O triângulo de Sierpinski aparece como atrator do sistema de funções iteradas $F=\left\{f_{1}, f_{2}, f_{3}\right\}$ dado por (B.5). 


\section{Índice Remissivo}

$\lambda$-Cálculo, 77

$\omega$-limite do sistema, 39

acoplamento, 16, 20

acoplamento bidirecional, 21

acoplamento mestre-escravo, 21

acoplamento unidirecional, 21

acoplamentos regulares, 22

amplitudes, 18

arestas, 50

autômatos celulares, 114

auto-organização, def., 48

auto-organização, 4, 27, 48

average path lenth, 51

baker's map, 12, 85, 87

Bernoulli shift map, 12

bias, 80

bifurcações, 10

branching functions, 85

breadth first search, 62

C.elegans, 54

cabeça de leitura, 74

cadeias de Markov, 77

caixeiro viajante, 105

caminho, 50

campo médio, 37

campo médio global, 37

campo vetorial, 8, 9, 18

caos, 12

cenas dinâmicas, 41

clusters, 1, 4, 26-28, 29, 48, 63, 64, 67-72

CML, def., 22

clustering, 26

clustering coefficient, 51

cobweb, 10

coeficiente de agregação, 51, 52, 54, 62, 65

completamente dessincronizado, 26 computação universal, def., 76

computação universal, 76, 77, 84

computabilidade, 73, 78, 102

condição de consistência, 75

condição de contorno periódica, 22

conjunto de Cantor, 82

conjunto invariante, 82

contra-domínio, 6

contração, 117

correlação, 35

coupled map lattices, def., 22

coupled map lattices, 4, 23, 29

degree distribution, 51

desordenado, 69

detecção de simetria, 4

diagramas de bifurcação, 10, 11

diagramas teia de aranha, 10

difeomorfismo, 9

dinâmica caótica, 12

dinâmica de uma função, 12

distância de fase, 63

distribuição de conexões, 51, 52

domínio, 6

drive-response, 21

eixos de reflexão, 31

emails, 49

equação de diferenças, 6

escala, 54

espaço celular, 114

expoente de distribuição, 53

fase, def., 18

feedback, 78

fluxos, 8, 9

freqüência angular, 18

freqüência natural, 18

função, 6

função de ativação, 80 
funções recursivas, 77

generalized shift map, 85

GCM, def., 23

globally coupled maps, def., 23

globally coupled maps, 4, 23

grafos, 50, 51

grafos não direcionados, 50

gramáticas estruturadas, 77

grau médio, 51

halt, 75

Heaviside, 80

hiperbólico, 117

homeomórfico, 47

hyperlinks, 49, 53

IFS, 117

interação fraca, 20

intermitente, 14

iterações, 6

iterated function systems, 117

L-sistemas, 89, 91-94, 96, 98, 99, 104, 106

labor networks, 49

laplaciano, 35

limiar, 80

LOGO, 90

máquina de Turing determinista, 74

máquinas baseadas em registradores, 77

mapa, 7

mapa de Poincaré, 9

mapa de retorno de Poincaré, 9

mapa do padeiro, 85,86

mapas, def., 19

mapas acoplados, 22, 59

mapas com funções de troca, 85

mapas de contração, 117

mapas lineares em intervalos, 81

mapas, sincronismo, 19

menor caminho médio, 51, 53, 62, 65

modelo de Watts-Strogatz, WS, 61

modelo Newman-Watts, NW, 62

modelo regular, CML, 61

modelo smallest-world, SW, 62

Newman-Watts, NW, 58

normas, 16 operações elementares, 87

operador laplaciano, 36

órbitas periódicas, 7

órbitas, def., 7

ordenado, estado, 26, 48

osciladores, 16

período, def., 18

piecewise linear maps, 81

Poincaré, mapa de, 9

ponto fixo, 7

pontos de bifurcação, 10

pontos periódicos, def., 7

probabilidade de religação, 57

procedimento efetivo, 77

rede, 51

redes aleatórias, 50

redes complexas, 49

redes neurais, 27

redes sem escala, 50, 55

redes small-world, 50

reduções de escala, 118

regra de transição, 114

ritmo, def., 18

ruído, 41

seção de Poincaré, 9, 10

shift map, 85

simetria, def., 30

simetria de reflexão, 31

simetria de rotação, 31

simetria, detecção de, 32

sincronismo, 16, 19, 63, 67

sincronismo de fase, 18

sincronização, 16

sincronização completa, 19

sistema acoplado, 20

sistema de mapas com função de troca, 85

sistema dinâmico discreto, 5

sistemas de regravação, 77

sistemas dinâmicos em tempo discreto, 5 , 6

sistemas estendidos, 22

small-world, 2-4, 53-61, 65-67, 69-72, 106

small-world efeito, 62, 65 
smallest-world, SW, 59

switching map systems, 85

tent map, 11

translações, 118

triângulo de Pascal, 118

triângulo de Sierpinski., 118

Turing, máquina de, 74, 81, 85 vértices, 50

verificador de paridade., 75, 76, 83

vizinhança de Moore, 24, 26

vizinhança de von Neumann, 24, 25

vizinhos, 50,54

Watts-Strogatz, WS, 57

world wide web, 49, 53 